\title{
2011/26
}

Equity in health and health care

Marc Fleurbaey and Erik Schokkaert

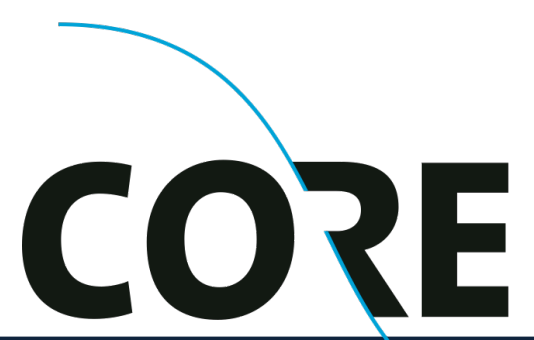

DISCUSSION PAPER

Center for Operations Research and Econometrics

Voie du Roman Pays, 34 B-1348 Louvain-la-Neuve Belgium http://www.uclouvain.be/core 


\title{
CORE DISCUSSION PAPER
}

$2011 / 26$

\section{Equity in health and health care}

\author{
Marc FLEURBAEY ${ }^{1}$ and \\ Erik SCHOKKAERT ${ }^{2}$
}

June 2011

\begin{abstract}
We discuss the conceptual foundations of measuring (in)equity in health and health care. After an overview of the recent developments in the measurement of socio-economic inequalities and in racial disparities, we show how these partial approaches can be seen as special cases of the more general social choice approach to fair allocation and equality of opportunity. We suggest that this latter framework offers many new analytical possibilities and is sufficiently rich to accommodate various ethical views. We emphasize that horizontal and vertical equity are intricately linked to each other. We then argue that a focus on overall well-being is necessary to put the partial results on health (care) inequity into a broader perspective and we discuss the pros and cons of various methods to evaluate the joint distribution of health and income: multidimensional inequality indices, dominance approaches, the use of happiness measures and finally the concept of equivalent income. Throughout the chapter the theoretical analysis is complemented with an overview of recent empirical results.
\end{abstract}

Keywords: equity, racial disparities, equality of opportunity, equivalent income, dominance.

JEL Classification: D630, I140, J150

${ }^{1}$ CNRS, Université Paris-Descartes, France; Université catholique de Louvain, CORE, B-1348 Louvain-laNeuve, Belgium. E-mail: marc.fleurbaey@parisdescartes.fr

${ }^{2}$ Department of Economics, K.U.Leuven, Belgium; Université catholique de Louvain, CORE, B-1348

Louvain-la-Neuve, Belgium. E-mail: erik.schokkaert@econ.kuleuven.be

We thank Chiara Canta, Tom McGuire, Tom Van Ourti and Fred Schroyen for their useful comments.

This paper presents research results of the Belgian Program on Interuniversity Poles of Attraction initiated by the Belgian State, Prime Minister's Office, Science Policy Programming. The scientific responsibility is assumed by the authors. 


\section{Introduction}

The economic literature on equity in health and health care has grown considerably since Wagstaff and van Doorslaer (2000a) published their survey chapter in Volume 1 of the Handbook of Health Economics. First, there has been an explosion of the number of empirical applications. Second, the different measures of inequity, including the popular concentration index, have come under theoretical scrutiny and there has been a lively debate on their normative implications. In this chapter we start from where Wagstaff and van Doorslaer (2000a) ended. We do not aim at giving a comprehensive survey of the empirical work and will mainly focus on the methodological and theoretical questions that are raised by the recent literature. However, as the ultimate aim of our approach is to define measures of inequity that can be brought to the data, we will assess how the different theoretical proposals can be implemented.

The first generation literature on socioeconomic inequality (as reviewed in Wagstaff and van Doorslaer 2000a) drew its inspiration mainly from the economic approach to the measurement of income inequality. However, this framework is not sufficiently rich to analyse all the relevant questions that have been raised in the recent literature. Why should we be concerned about health (care) inequalities? More specifically, when are such inequalities a cause of concern and when are they ethically legitimate? And what implications do the answers on these questions have for the measurement of inequity? How to integrate different dimensions of inequity? And what is the place of health (care) in a broader concept of well-being? We will try to push these ongoing developments a bit further by exploring the potential contribution to health economics of modern social choice theory. Our approach is an attempt to integrate broader philosophical questions, measurement issues and data considerations.

There are obvious limitations to this chapter. We will focus on inequity in health and in health care and only briefly refer to the literature on equity in finance. This is not only for reasons of space, but also because we believe that the issue of equity in finance can only be analysed in a meaningful way within a broader welfare framework. We argue this in greater detail in section 5. Moreover, we are focusing on conceptual and measurement issues regarding equity (or justice). We are not treating incentive issues and we will therefore not discuss the equity-efficiency trade-off. Finally, we can only touch the voluminous literature outside economics (e.g., in public health). This is not to suggest that economists necessarily should have the last word in the debate. The approaches from different disciplines are complementary, but we want to describe what could be the specific contribution of economics to this joint venture. In our view, a crucial advantage of the economic approach is the development of a strong formal framework 
of analysis. Presenting such a formal framework is our main purpose.

Merely describing and explaining differences in health and in health care is a positive question, but measuring and evaluating what is inequitable in the given situation is a normative topic. Indeed, the evaluation of a given state of affairs requires value judgments as well as analysis of empirical data. Some might claim that answering such normative questions is not part of economic science. Yet, if economists refuse to address these crucial questions, who will do it for them? In his seminal monograph "Collective choice and social welfare", Sen (1970) introduced a distinction between two sorts of value judgments. Basic value judgments are purely philosophical statements. Kant's categorical imperative is an example. Yet, when evaluating economic situations such as the degree of inequity in health (care), what is involved are non-basic value judgments. These are contingent on empirical facts (or, at least, on a given interpretation of observable reality). Indeed, they crucially depend on the definition of relevant empirical concepts. We believe that the social sciences can and should make a contribution to the elaboration of such non-basic value judgments.

We will illustrate in the next section the intricate relationship between data and normative statements on equity in health and health care with a specific example. This will give us the opportunity to introduce some of the issues that will be discussed later in this chapter.

\section{An example}

Health and health care obviously are unequally distributed over the population. Some older papers focused on health inequality as such (Le Grand 1987), but one can doubt whether all health inequalities are per se inequitable. Some determinants of health (such as age) cannot be influenced by policy if the health inequalities in two countries differ because of differences in the age composition of their population, few would see this as an indication of inequity. Moreover, a similar question may be asked if the inequalities in health reflect only differences in the lifestyle choices of populations. It seems therefore that not all health inequalities are inequitable. This is even more obvious for inequalities in health care use. Almost everybody agrees that differences in use of care which reflect differences in health status may be justified. Surely, therefore, a correction for needs is necessary before we can talk about inequity. The crucial question is how this correction should be performed. All this suggests that evaluating inequity in health and health care requires two steps. First, one has to see which factors explain the observed inequality in health (care). Second, one has to take a normative position on whether differences due to 
each of these factors can be ethically justified.

Let us illustrate the issue by looking in some detail at an example of a (very detailed) empirical study. Morris et al. (2005) investigate the inequity in the use of health care in the English NHS. The first step in their analysis is to estimate the empirical relationship between access to health care on the one hand and individual characteristics on the other hand. They pool data $(1998,1999,2000)$ from the Health Survey for England for a total sample size of 50,977 individuals. These data contain all the usual demographic and socioeconomic information, but, more importantly, also exceptionally rich health information at the individual level (not only subjectively assessed health, but in addition information about types of longstanding illnesses and even GHQ-12 scores). They analyse the effects of all these characteristics on four use variables: a first variable indicating whether individuals had a GP consultation in the last two weeks, and three other indicators for having had an outpatient visit, day case treatment or an inpatient stay in the previous 12 months. After some statistical testing, they opt for a linear probability model in the first two cases, and for a probit model in the last two. Everything taken together, the estimated model contains over 200 explanatory variables. In Table 1 we summarize the significant effects for groups of variables, where significance is defined as $p \leqslant 0.0001 .{ }^{1}$ If no comment is added to the table, the effect of the variables goes in the expected direction (e.g., healthier people consuming less care).

The many significant coefficients clearly indicate that there is inequality in the use of health care, in that different individuals consume different amounts of care. But what about inequity? As mentioned before, we surely have to control for differences in health status. Indeed, the most common interpretation of horizontal inequity in this context is that two individuals with the same morbidity receive different amounts of care. In this interpretation, the message of Table 1 seems clear. Since, after controlling for a large set of health variables, socioeconomic and ethnic variables still influence health care use, there was horizontal inequity in the NHS in the period 1998-2000. This is further corroborated by the significant supply effect in the regression for day care treatment. Yet some tricky questions remain. Socioeconomic and ethnic variables may capture to some extent differences in preferences. Are differences in use inequitable if they simply reflect choices by the patients? Is it possible to identify with real-world data differences in preferences versus differences in needs?

\footnotetext{
${ }^{1}$ This strict criterion is chosen because of the very large sample size.
} 
Table 1. Explaining health service utilization (Morris et al. 2005)

\begin{tabular}{|c|c|c|c|c|}
\hline & GP consultations & Outpatient visits & Day case treatment & Inpatient stays \\
\hline Age and sex & significant $^{a}$ & significant $^{a}$ & significant $^{a}$ & significant $^{a}$ \\
\hline Self-reported general health & significant & significant & significant & significant \\
\hline Acute ill health & significant & & & \\
\hline Ward-level health variables & significant $^{b}$ & ns & ns & ns \\
\hline Type of longstanding illness & significant & significant & significant & significant \\
\hline Number of longstanding illnesses & ns & significant & significant & ns \\
\hline GHQ-12 score & significant & significant & significant & significant \\
\hline Income & ns & significant $^{d}$ & ns & ns \\
\hline Social class & ns & ns & ns & ns \\
\hline Economic activity $^{c}$ & significant $^{e}$ & significant $^{f}$ & significant & significant $^{f}$ \\
\hline Education & ns & ns & ns & ns \\
\hline Ethnic group & ns & significant $^{g}$ & ns & ns \\
\hline Supply effects & ns & ns & significant $^{h}$ & ns \\
\hline
\end{tabular}

COMMENTS:

a) curvilinear relationship with age, depending on gender and on type of health care; b) individuals living in a "healthier" environment have less GP consultations; c) (overall) significant effects of going to school, permanent long-term sickness, retired, temporary sickness: suggest that health variables are not sufficient; d) high income people have a larger probability of outpatient visits; e) individuals looking for paid work have less GP consultations; f) individuals looking after the home have less outpatient visits and more inpatient stays; g) less outpatient visits for Pakistani, Bangladeshi, Chinese ethnic groups; h) more day case treatment when more GP's per 1000 patients.

Suppose we have reached agreement about these questions and accept that the significant effects found for many non-need variables indicate that there is horizontal inequity in the system. There then still remains the methodological question of deriving a measure that would allow us to rank different situations with respect to the degree of horizontal inequity. What weight should be given to the different components of inequity? As the example shows, there are two different issues here. First, the pattern of inequity is different for different health care items. How to aggregate these? Can the fact that people from Pakistani origin go more often to the GP compensate for the fact that they have a smaller probability of outpatient visits? Simply taking total health care expenditures may be a very rough indicator. Second, 
how to bring together different forms of inequity, e.g., socioeconomic inequality, ethnic disparities and regional (supply-side) differences? Focusing on one of these forms leads to partial indicators, which may be very useful in their own right, but raises also new questions. Suppose we want to measure socioeconomic inequity in health care use. Should we derive our measure from a regression including all other variables as controls? Assume (realistically) that there is a positive relationship between supply and socioeconomic status (SES) of individuals, in that lower SES individuals live in regions with more limited supply. Would it then still be acceptable to control for supply side differences? Or should we include them in our measure of socioeconomic inequity? If yes, how?

Until now we focused on horizontal inequity. Another issue is the one of vertical equity, which would be attained when "individuals with different levels of need consume appropriately different amounts of health care" (Morris et al. 2005, p. 1251). It is clear that estimation results such as the ones summarized in Table 1, only show the observed relationship between health status and health care use, and that there is no guarantee at all that this observed relationship is equitable. The problem of vertical equity is underexplored in the empirical literature, mainly because it requires answering the vexing question of what are "appropriate different amounts of health care" (but see Sutton 2002). This may seem an almost unsolvable medical question, but things are even worse: giving an appropriate answer requires going beyond the health setting in the narrow sense, as it unavoidably raises the issue of the optimal trade-off between health and other dimensions of well-being. Surely, in a world with scarce resources, defining appropriate amounts of health care at different levels of need cannot boil down to giving everybody the best care that is technically possible.

The Morris et al. (2005) example focused on inequity and inequality in the use of health care, but it should be clear that analogous questions arise with respect to inequality and inequity in health. We mentioned already that health inequalities are also influenced by a host of different factors, some of which may be seen as perfectly legitimate. Moreover, health can also be measured on different dimensions. In fact, measuring health is more difficult than measuring health care use and we will see that this may complicate the evaluation of the degree of inequity. Finally, a broader perspective on health inequity certainly forces us to look at the broader picture of overall well-being.

All the questions that we have introduced here have cropped up in the recent literature on inequity in health and health care. This will become clear in Section 3, in which we give an overview of the most popular "partial" approaches, focusing on socioeconomic inequities and on racial disparities. We will then introduce in section 4 the social choice approach to fair allocation and equality of opportunity 
and show how it may offer a theoretically integrated framework to answer these various questions in a coherent way. Section 5 focuses on the broader relationship between health care, health and well-being and about its implications for measuring inequity. Section 6 concludes.

\section{Socioeconomic inequity and racial disparities in health and health care}

The example in the previous section suggests that inequity in health (care) is inherently a multidimensional phenomenon. However, up to now, the literature has focused on partial indicators which are specifically meant to measure the influence of a certain individual or group characteristic (income, socioeconomic status, race, gender) on some variable of interest (health, health care). Such a partial approach requires less information than a complete multidimensional approach. More importantly, it can also be justified with the argument that policy makers and citizens seem to be strongly interested in these more specific questions. Two topics have been particularly salient. In subsection 1 we discuss the booming literature on "socioeconomic" inequities in health (care), that has been dominated by the concept of the concentration index. In subsection 2 we introduce the issue of racial (or gender) disparities. Since race and gender are discrete variables, the concentration curve approach cannot be applied and other methods have been proposed and implemented.

\subsection{Socioeconomic inequalities in health and health care: the concentration curve and index}

The concentration curve and the concentration index have become the workhorses for a large and rapidly growing empirical literature on socioeconomic inequalities in health and health care. Papers measuring concentration dominated the chapter by Wagstaff and van Doorslaer (2000a) and more recent developments are the topic of the recent survey by van Doorslaer and Van Ourti (2011). The World Bank has published a practical guide, covering the most important theoretical issues related to the approach with a focus on implementation (O'Donnell et al. 2008). We will not duplicate these comprehensive surveys and rather cover the main methodological developments and questions. This will pave the way for the introduction of the equality of opportunity approach in section 4. We first give in subsection 3.1.1 a brief overview of the main normative presuppositions behind the concentration index. We will then comment 
(subsection 3.1.2) on the measurement issues that are related to the specific nature of the health variables. Finally we will argue in subsection 3.1.3 that the decomposition of the overall effect, which has become rather popular in recent applications, confronts us directly with the questions previewed in the previous section.

\subsubsection{Principles}

Let us briefly recall the basic rationale for the concentration index as a measure of socioeconomic inequality in health and health care. In the light of the ideas from the previous section, this is a partial approach aiming at measuring only the inequity resulting from inequalities between different socioeconomic groups. The measure can be applied to any indicator of socioeconomic status, provided it is possible to rank the individuals unambiguously from low to high SES. In fact, different indicators may give different results and the choice between them is an open issue that we will leave aside in this chapter. For convenience, we focus on income $I$. Let us denote the relevant outcome by $y$ (we will use $h$ when we refer exclusively to health and $h c$ when we refer exclusively to health care). As an example, Figure 1 (taken from Wagstaff and van Doorslaer 2000b) shows a concentration curve for medical care $L_{M}(R)$, plotting the cumulative proportion of medical care against the cumulative proportion of population ranked by socioeconomic status (here $I$ ). If the concentration curve coincides with the diagonal, there is no socioeconomic inequality. If the curve lies below (above) the diagonal there is inequity in favor of the better-off (worse off). Inequity in favor of the rich means that the rich have relatively better outcomes than the poor.

It is clear that the distance between the concentration curve and the diagonal gives us an idea about the degree of inequity. This intuition is captured by the concentration index (CI), which is defined as twice the area between the concentration curve and the diagonal, or

$$
C I(y)=1-2 \int_{0}^{1} L_{y}(r) d r
$$

With a discrete number of individuals $n$, this can be shown to be equal to:

$$
C I(y)=\frac{1}{n \mu(y)} \sum_{i=1}^{n}\left[y_{i}\left(2 r_{i}^{I}-\frac{n+1}{n}\right)\right],
$$

where $\mu(y)$ is the average value of $y_{i}$ and $r_{i}^{I}$ is the fractional rank of individual $i$ in the distribution of $I$. The concentration index $C I(y)$ lies between -1 and +1 . It takes positive (negative) values when the concentration curve lies below (above) the diagonal. This means that a positive (negative) value corresponds to inequalities favoring the rich (poor). Note that the concentration curve can cross the 


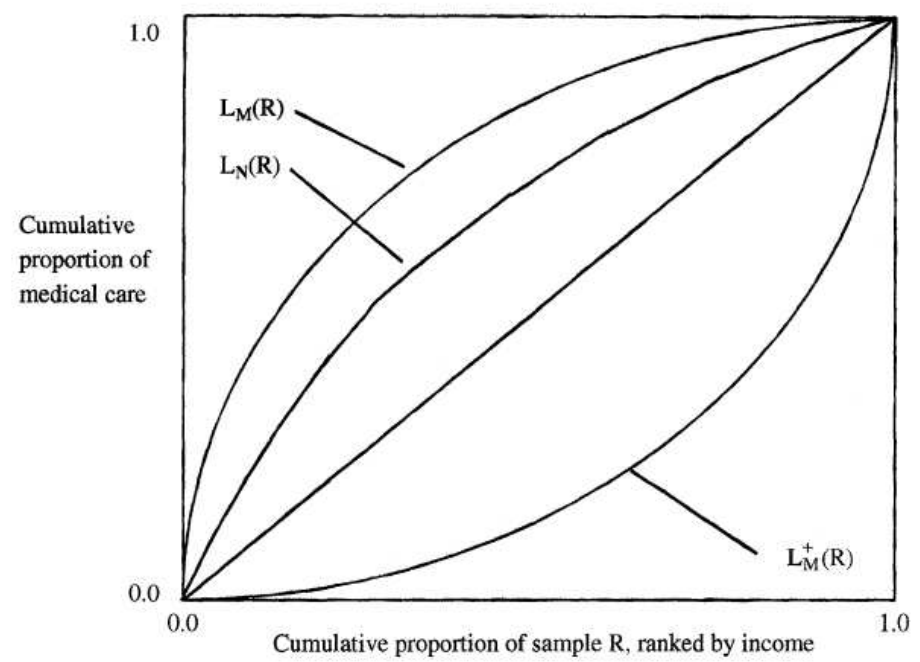

Figure 1: Concentration curves of medical care - direct and indirect standardization

diagonal. Therefore $C I(y)$ can be 0 , even if there are socioeconomic inequalities, provided inequalities in different parts of the distribution go in different directions and exactly compensate each other.

As (2) shows, the concentration index depends mainly on the covariance between $y_{i}$ and $r_{i} .{ }^{2}$ It is therefore proportional to the coefficient of the linear regression of $y_{i}$ on $r_{i}^{I}$. More precisely, regressing the function

$$
\frac{2 \sigma_{r}^{2}}{\mu(y)} y_{i}=\alpha+\beta r_{i}^{I}+\varepsilon_{i}
$$

where $\sigma_{r}^{2}$ is the variance of $r_{i}^{I}$, one obtains an estimated $\hat{\beta}$ which is by construction equal to the concentration index (Kakwani et al. 1997). This is a convenient way to calculate the concentration index and to check its statistical properties.

This approach can be used immediately to measure socioeconomic inequalities in health $(h)$, and the concentration index will then measure inequity if one accepts that all such socioeconomic health inequalities are illegitimate. We will return to this assumption later on, but let us for the moment assume that they are. ${ }^{3}$ As argued before, however, for medical care hardly anybody thinks that it should be equal for all citizens. Consider again the concentration curve for medical care $L_{M}(R)$ in Figure 1 . The curve shows that the poor consume relatively more health care, but this is not necessarily inequitable if

\footnotetext{
${ }^{2}$ Inspired by Milanovic (1997), Koolman and van Doorslaer (2004) show that the concentration index depends on the coefficient of variation of $y$ and on the correlation between $y$ and the fractional rank.

${ }^{3}$ Note that socioeconomic inequality in health (as measured by the concentration index) does not coincide with inequality in health. See, e.g., Wagstaff and van Doorslaer (2004).
} 
they are more needy. Two approaches have been proposed in the literature to implement a correction for differences in health status (Wagstaff and van Doorslaer 2000). Let us assume for simplicity that we can write health care as a function of needs $N$ and socioeconomic status $S E S$, i.e., $h c_{i}=f\left(N_{i}, S E S_{i}\right)$, neglecting all other variables. The first method is direct standardization, by which one calculates for each individual how much medical care she would have received if she had been in the same degree of need as the sample as a whole, i.e., $h c_{i}^{+}=f\left(\bar{N}, S E S_{i}\right)$, where $\bar{N}$ is average need in the sample. These recalculated health care levels $h c^{+}$then can be used to construct the concentration curve $L_{M}^{+}(R)$ in Figure 1 and to calculate the corresponding concentration index $C^{D I R}(h c)=C\left(h c^{+}\right)$. The second method is indirect standardization. This considers the hypothetical situation indicating for each individual the amount of medical care she would have received if she had been treated like others with the same need characteristics, i.e., $f\left(N_{i}, \overline{S E S}\right)$, where $\overline{S E S}$ is average SES in the sample. The "inequity" suffered by individual $i$ can then be measured as $f\left(N_{i}, S E S_{i}\right)-f\left(N_{i}, \overline{S E S}\right)$. In terms of the concentration curves, the overall evaluation will be based on a comparison of $L_{M}(R)$ with the needs concentration curve $L_{N}(R)$, where the latter is the concentration curve of $f\left(N_{i}, \overline{S E S}\right)$, i.e., of indirectly standardized medical care. The relevant concentration index $C^{I N D}(h c)$ is twice the area between $L_{N}(R)$ and $L_{M}(R)$ and all the formulae can be adjusted in a straightforward way.

The CI-approach is a convenient way to measure socioeconomic inequity in health and health care. Of course, it is only intended to be a partial approach, neglecting other relevant inequities in society. Taken this limitation for granted, what are its main normative presuppositions? First, defining inequity as twice the area between the concentration curve and the diagonal implies a specific weighting pattern. As (2) shows, $C I(y)$ is a weighted sum of shares with weights equal to $2 r_{i}^{I}-\frac{n+1}{n}$. Although these weights are also those of the popular Gini index of income inequality, they remain arbitrary. Therefore, Wagstaff (2002) proposed a natural generalisation of $C I(y)$, which is analogous to the extended Gini index:

$$
E C I(y)=\frac{1}{n \mu(y)} \sum_{i=1}^{n}\left\{y_{i}\left[1-\nu\left(1-r_{i}^{I}\right)^{\nu-1}\right]\right\}
$$

where the parameter $\nu$ captures inequality aversion. If $\nu=1$, there is no inequality aversion and $E C I(y)=0$. For $\nu=2$, we recover the standard concentration index in the case of large $n$. This extension has not been frequently used in the applied literature.

Second, deeper doubts with respect to both the concentration index and its generalisations have been brought to the fore by Fleurbaey (2006) and Bleichrodt and Van Doorslaer (2006). Focusing on 
socioeconomic inequality in health (rather than in health care), Bleichrodt and van Doorslaer (2006) show that the social welfare function underlying the generalized concentration index is characterized by a set of conditions, including anonymity and what they call the principle of income-related health transfers. ${ }^{4}$ Anonymity implies that the only characteristics that are allowed to influence social welfare judgments are the individual's health and her rank in terms of SES. This reflects the partial nature of the approach, but in this explicit formulation it is obviously very restrictive. The principle of income-related health transfers states that transferring health from someone who is better off in terms of SES to someone who is worse-off in terms of SES does not lead to a reduction in social welfare, provided the transfer does not change the ranking of the individuals in terms of SES. As Bleichrodt and van Doorslaer (2006, p. 955) emphasize, this principle is not very plausible. "It does not seem desirable to transfer health from a person with high living standards to a person with lower living standards when the person with high living standards is in poor health and differences in living standards are small." In fact, both conditions throw a sharp light on the limitations of the CI approach. These issues will be taken up again in sections 4 and 5. For the moment, we will remain within the CI framework.

\subsubsection{Measurement issues}

As mentioned before, the original work on the CI took its inspiration mainly from the literature on measuring income inequality. Income is an unbounded variable that is measured at the ratio level. The same is true for health care expenditures and the whole apparatus of income inequality measurement can therefore be applied to health care without much of a problem. However, the measurement of health is less clear and recently there has been some debate on how to take account of the measurement characteristics of the health variable within the framework of the CI approach. ${ }^{5}$

The CI approach should only be used with variables measured on a ratio scale, i.e., when there is a natural zero. Take the case of SAH (subjectively assessed health), which has been a popular health indicator in applied work. Since this is a categorical measure, authors have tried in the past to map a

\footnotetext{
${ }^{4}$ Other less disputable conditions used in the characterization are 1) monotonicity (if everybody's health increases, social welfare increases), 2) the existence for each health profile of an equally distributed equivalent level of health and 3) additivity (take three ordered health vectors $h, h^{\prime}$ and $h^{\prime \prime}$. Then $h$ is socially preferred to $h^{\prime}$ if and only if $h+h$ " is socially preferred to $\left.h^{\prime}+h "\right)$. To characterize the CI (rather than the ECI) the principle of income-related health transfers has to be strengthened further to get at the specific weighting structure embodied in (2).

${ }^{5}$ Note that for application of the CI, it is sufficient to rank all individuals according to SES, i.e., for the SES-variable measurement at the ordinal level is sufficient.
} 
cardinal scale on the categories. A good example is the paper by van Doorslaer and Jones (2003). They estimate an ordered probit model with SAH as the dependent variable and specify the underlying latent variable $y_{i}^{*}$ as a function of a vector of socioeconomic characteristics, i.e., $y_{i}^{*}=x_{i} \beta+\varepsilon_{i}$ with $\varepsilon_{i} \sim N(0,1)$. They then use as a measure of individual health the predictions of the linear index $x_{i} \beta$ after rescaling. However, as emphasized by Erreygers and Van Ourti (2010), this rescaling procedure is not innocuous, because the calculated CI will depend on the chosen zero point. If there is a natural zero point, health can be measured on a ratio scale and there is no problem. If there is no natural zero point, one should check the robustness of one's conclusions, e.g., for some comparison of distributions, with respect to the choice of zero. If the conclusions remain the same for all plausible values of the zero, there is no need to worry. Otherwise, it is necessary to specify exactly the range for which they hold.

Things get more complicated if the range of the health variable is bounded, i.e., if $h^{\min } \leqslant h_{i} \leqslant h^{\max }$ where both $h^{\min }$ and $h^{\max }$ are finite. Wagstaff (2005) noted that for binary health indicators (e.g., surviving yes or no), the theoretical bounds of the CI depend on the bounds of the health variable (in the example 0 and 1) and on the mean. This complicates the comparison of the results for different populations. Clarke et al. (2002) pointed to another issue. For any bounded health variable it is natural to define a corresponding ill-health variable as the shortfall

$$
s_{i}=h^{\max }-h_{i} .
$$

Take as an example chronic malnutrition of children as measured by stunting. We then can define either an individual 0-1 health variable taking the value 1 if the child is not stunted, or a $0-1$ ill-health variable taking the value 1 if the child does suffer from chronic malnutrition. The choice between the two is essentially arbitrary, and it seems desirable that the evaluation of socioeconomic inequality would be consistent whether working with health or with ill-health. However, the results with the CI will be sensitive to this choice.

These problems were taken up and analysed in an integrated approach by Erreygers (2009a,b) and Erreygers and Van Ourti (2010). They focus exclusively on the family of rank-dependent measures for bounded variables, represented by

$$
I(y)=f\left(y^{\min }, y^{\max }, \mu(y), n\right) \sum_{i=1}^{n} z_{i} y_{i}
$$

where $z_{i}=n\left(r_{i}^{I}-\frac{n+1}{2 n}\right), y=h, s$ and $f($.$) is a continuous function. If y$ is unbounded, $f($.$) reduces to$ $f(\mu(y), n)$. It is easy to see that $C I(h)\left(=\left[2 /\left(n^{2} \mu(h)\right)\right] \sum_{i=1}^{n} z_{i} h_{i}\right)$ is a special case of (6). Erreygers and 
Van Ourti (2010) argue that an attractive rank-dependent index of socioeconomic inequality in health $I(y)$ should satisfy at least two conditions. The first is scale invariance: the index should be invariant to positive affine (linear) transformations of the distribution of health, if the latter is measured on a cardinal (ratio) scale. The second one is the mirror condition. If $h$ is a given health distribution and $s$ its associated ill-health distribution, then applying the index should yield consistent results in both cases, i.e., $I(h)=-I(s) .{ }^{6}$ It is then shown that the following parametric subclass of the rank-dependent indices (6) satisfies both conditions:

$$
I^{\theta}(h)=\frac{8}{n^{2}\left[4 \mu\left(h^{*}\right)\left(1-\mu\left(h^{*}\right)\right]^{\theta}\right.} \sum_{i=1}^{n} z_{i} h_{i}^{*},
$$

where $h_{i}^{*}=\left(h_{i}-h^{\min }\right) /\left(h^{\max }-h^{\min }\right)$. Note that the CI and the ECI are not included in the family (7). Indeed, the CI does satisfy neither scale invariance nor mirror, the ECI is not scale invariant. The family does include a generalisation of the proposal made by Wagstaff (2005) for $\theta=1$. It also includes the index proposed by Erreygers $(2009 \mathrm{a}, \mathrm{b})$ himself for $\theta=0$.

The interpretation of the parameter $\theta$ is essential. Erreygers (2009b) and Erreygers and Van Ourti (2010) show that a scale-invariant rank-dependent index cannot satisfy at the same time the mirror property and measure only relative inequality, i.e., satisfy the condition that $I(y)=I(r y)$ when $0<r<$ 1. They then suggest that it is desirable for a rank-dependent index to give some weight to absolute inequality, i.e., to satisfy the condition that, for $0<r<1$, (i) if $I(y)>0$, then $0<I(r y)<I(y)$; (ii) if $I(y)<0$, then $I(y)<I(r y)<0$; and (iii) if $I(y)=0$, then $I(r y)=I(y)$. For the parametric class of indices (7), absolute inequality will matter if $0 \leqslant \theta \leqslant 1$. The Wagstaff and Erreygers measures therefore are at the two extremes. For both a reasonable interpretation can be given.

Erreygers (2009b) and Erreygers and Van Ourti (2010) propose to impose a convergence property stating that $\lim _{r \rightarrow 0} I(r h)=0$ (for $h$ being a given distribution). For the parametric class in (7), this property will only hold if $\theta<1$, i.e., it does not hold for the Wagstaff index. Moreover, if one imposes also a linearity condition $(I(r y)=r I(y)$ for $0 \leqslant r<1), \theta$ has to be equal to 0 , i.e., one ends up with

\footnotetext{
${ }^{6}$ The distinction between "achievement" and "shortfall" inequality is also relevant in the context of pure (rather than socioeconomic) health inequality. In this context, Erreygers (2009c) derives two inequality indices - variations of the absolute Gini coefficient and the coefficient of variation — that satisfy his "mirror" condition that inequality of attainments and inequality of shortfalls are cardinally identical, i.e. $I(h)=-I(s)$. This mirror condition is very strong and a more natural "consistency" requirement would be that the ranking of distributions of $h$ should be the same as the ranking of distributions of $s$. However, Lambert and Zheng (2011) show that (for a much larger class of inequality indices) all indices satisfying this weaker consistency condition also satisfy Erreygers's strong mirror condition.
} 
the Erreygers index. The normative justification for this linearity condition is not very clear, however. Moreover, one can also wonder whether the convergence property is necessarily appealing. Is there really a problem with a discontinuity at $r=0$ ? Wagstaff (2009) argues in favor of his own normalization on the basis of the following hypothetical example. Suppose for convenience that maximal health involves a health score of 1 and minimal health a score of 0 . Suppose that in the initial situation the mean is 0.1 and that there are 100 individuals involved. The most pro-rich distribution possible is one where the richest 10 people have a health score of 1 and all the others have a health score of 0 . Now suppose that mean health increases to 0.2 . Then the most pro-rich health distribution would be one where the richest 20 people have health 1 and everyone else has zero health. Since in both these distributions maximum inequality is reached, it seems natural to say that they are just as unequal from a pro-rich perspective and should therefore give the same value to the index. The Wagstaff index $(\theta=1)$ satisfies this intuition, the Erreygers index $(\theta=0)$ does not.

Table 2. Socioeconomic inequalities in stunting, calculated with different measures

\begin{tabular}{|l|l|l|l|l|l|}
\hline Country & $\mu(s)$ & $-C(s)$ & $C(h)$ & $W(h)$ & $E(h)$ \\
\hline Malawi 2000 & 0.4902 & 0.0756 & 0.0727 & 0.1483 & 0.1482 \\
\hline Ethiopia 2000 & 0.4730 & 0.0390 & 0.0350 & 0.0740 & 0.0738 \\
\hline Cameroon 2004 & 0.3165 & 0.1698 & 0.0786 & 0.2484 & 0.2150 \\
\hline Guinea 1999 & 0.2607 & 0.1067 & 0.0376 & 0.1443 & 0.1113 \\
\hline Haiti 2000 & 0.0797 & 0.2154 & 0.0187 & 0.2341 & 0.0687 \\
\hline Paraguay 1990 & 0.0394 & 0.3216 & 0.0132 & 0.3348 & 0.0507 \\
\hline Colombia 2005 & 0.0215 & 0.2699 & 0.0059 & 0.2758 & 0.0232 \\
\hline
\end{tabular}

Countries are ranked in decreasing order of the prevalence of stunting $(\mu(s)$ being the mean). Stunting is a dummy variable. The different columns correspond respectively to minus the concentration index of stunting $(-C(s))$, the concentration index of non-stunting $(C(h))$, the Wagstaff index for non-stunting $(W(h))$ and the Erreygers index for non-stunting $(E(h))$.

Ultimately (and not surprisingly) the choice between the different indices boils down to an ethical choice between different normative ideas. That the choice does matter, however, is illustrated in Table 2 (which is a selection taken from from Table 1 in Erreygers 2009a). The Table gives the results for 
socioeconomic inequality in stunting as calculated with different indices for various countries and time periods. If the prevalence of stunting is close to the middle of $h^{\min }$ and $h^{\max }, C(h)$ and $-C(s)$ are close to each other, and the same is true for the Wagstaff index $W(h)$ and the Erreygers index $E(h)$. However for countries with a low prevalence of stunting the Wagstaff index gives much larger values than the Erreygers index (and is in effect close to $-C(s)$ ). This is of course the empirical illustration of the convergence properties that have been described before. Choosing a specific member of the family of rank-dependent indices (6) is not without consequences.

\subsubsection{Developments in the empirical literature}

As the measurement issues raised in the previous section have only appeared in the literature recently, they have not yet had much influence on applied work. This applied literature is rapidly growing. As van Doorslaer and Van Ourti (2011) have given a comprehensive overview of empirical results and of policy implications, we will only focus on what seem to be three important developments.

First, while the empirical applications of the CI approach initially focused on European and NorthAmerican countries, there is now also an extensive coverage of developing countries (see, among others, Cisse et al. 2007, Lu et al. 2007, O'Donnell et al. 2008, Schneider and Hanson 2006, Van de Poel et al. 2007, Wagstaff 2000). The specific features of these countries have led to a growing interest in the link between health care and poverty. We will return to this link in section 5 .

Second, from a methodological perspective, it is worth pointing to the growing popularity of decomposition methods. Wagstaff et al. (2003) have shown that, if the relevant outcome $y$ can be written as a linear function of a set of characteristics $x$, i.e.

$$
y_{i}=\beta_{0}+\sum_{j=1}^{K} \beta_{j} x_{i j}+\varepsilon_{i}
$$

then the concentration index $C I(y)$ can be decomposed as

$$
C I(y)=\sum_{j=1}^{K} \eta_{j} C I\left(x_{j}\right)+\frac{2 \operatorname{cov}\left(\varepsilon_{i}, r_{i}^{I}\right)}{\mu(y)}
$$

where $\eta_{j}=\beta_{j} \mu\left(x_{j}\right) / \mu(y)$. Equation (9) shows that the effect of any variable $x_{j}$ on $C I(y)$ will depend both on its own concentration index (or, if $x_{j}$ is the SES indicator itself, its Gini coefficient) and on the "elasticity" $\eta_{j}$ of $y$ with respect to $x_{j}$.

This decomposition approach has been used to interpret differences in the concentration index between different countries or changes over time. ${ }^{7}$ Indeed, the linear structure of (9) makes it possible to

\footnotetext{
${ }^{7}$ Jones and Lopez-Nicolas (2004) have used a similar approach to decompose long run socioeconomic inequality as a
} 
decompose changes and differences in the concentration index as in Oaxaca (1973) and Blinder (1973). Neglecting for convenience the last term in (9), the difference between two situations 1 and 2 can be written as

$$
C I^{1}(y)-C I^{2}(y)=\sum_{j=1}^{K} \eta_{j}^{1}\left[C I^{1}\left(x_{j}\right)-C I^{2}\left(x_{j}\right)\right]+\sum_{j=1}^{K} C I^{2}\left(x_{j}\right)\left[\eta_{j}^{1}-\eta_{j}^{2}\right]
$$

Good examples for respectively health and health care are van Doorslaer and Koolman (2004) and van Doorslaer et al. (2004), both making use of data from the European Community Household Panel. In the case of socioeconomic inequalities in health, van Doorslaer and Koolman (2004) show that these are particularly high in Portugal, the UK and Denmark. Demographic variables are part of the "explanation", but other important factors are income, education, labor force status and region. The somewhat surprising result for Denmark is ascribed to the fact that the early retired in Denmark have much worse health and are strongly concentrated among the low income groups. As to income-related inequalities in doctor utilisation, van Doorslaer et al. (2004) point out that there is substantial pro-rich inequity in virtually every country with respect to the probability of seeing a medical specialist. Income, education and region are important contributing factors. While these decomposition results give interesting insights into the pattern of socioeconomic inequalities, they should be interpreted cautiously. More specifically, the titles of both papers suggest that the decompositions give an "explanation" of the socioeconomic differences. This is misleading, however, as it mistakenly suggests that one can give a causal interpretation to (8).

The decomposition approach has also been used to tackle the standardization problem. As mentioned before, socioeconomic inequalities in health care as such are not worrying, if they reflect differences in needs. Therefore a needs correction is needed. In a similar spirit, it is common practice to standardize the health data for demographic factors before measuring socioeconomic inequalities in health, under the supposition that health differences due to age and gender do not raise an equity issue. The decomposition approach makes it possible to calculate "partial concentration indices" by putting some of the components at the RHS of (9) equal to zero (Gravelle 2003). Let us illustrate for socioeconomic inequalities in health care. Suppose that we can partition the vector $x_{i}$ between needs (or standardizing) variables $n_{i}$ and non-needs variables $z_{i}$, so that we can rewrite (8) as

$$
h c_{i}=\beta_{0}+\sum_{j=1}^{L} \beta_{j}^{n} n_{i j}+\sum_{j=1}^{M} \beta_{j}^{z} z_{i j}+\varepsilon_{i},
$$

As described before, the direct standardization method puts the needs factors in (11) at a fixed weighted sum of short-run concentration indices (and a term capturing mobility). See Allanson et al. (2010) for a further development along this line. 
value, which yields

$$
h c_{i}^{+}=\beta_{0}+\sum_{j=1}^{L} \beta_{j}^{n} \bar{n}_{j}+\sum_{j=1}^{M} \beta_{j}^{z} z_{i j}+\varepsilon_{i}
$$

and, therefore,

$$
C I^{D I R}(h c)=C I\left(h c^{+}\right)=\sum_{j=1}^{M} \eta_{j}^{z} C I\left(z_{j}\right)+\frac{2 \operatorname{cov}\left(\varepsilon_{i}, r_{i}^{I}\right)}{\mu(h c)}
$$

In the case of indirect standardization, one first calculates a "corrected" value for health care by putting the non-needs variables in (11) at a fixed value and one then focuses on the differences between actual health care levels and these "corrected" health care levels. ${ }^{8}$ In terms of the concentration index this yields

$$
C I^{I N D}(h c)=C I(h c)-\sum_{j=1}^{L} \eta_{j}^{n} C I\left(n_{j}\right)=\sum_{j=1}^{M} \eta_{j}^{z} C I\left(z_{j}\right)+\frac{2 \operatorname{cov}\left(\varepsilon_{i}, r_{i}^{I}\right)}{\mu(h c)}
$$

It turns out that the direct and indirect standardization methods in this case coincide. This is due to the additive separability between the variables $n_{i}$ and $z_{i}$, that is implied by the linearity assumption in (11). Direct and indirect standardization will give different results if there are what Gravelle (2003) calls essential nonlinearities, i.e., if the marginal effect on health care of the $z$ variables depends on the values taken by the needs variables (Gravelle 2003). The easy decomposition approach, however, crucially hinges on the linearity assumption.

Third, on the econometric side, panel data have been used to capture unobserved heterogeneity. There has been a move towards the estimation of complex non-linear models that are better able to capture the specific nature of the dependent variables (e.g., a random effects count data model in Van Ourti 2004, or a latent class hurdle model in Bago d'Uva et al. 2009). The simple linear decomposition methods can then no longer be applied. Approximation methods have been proposed, but these are (by definition) only approximations and considerably complicate the interpretation of the error term (van Doorslaer et al. 2004).

In the literature on the CI, these issues have sometimes been seen as statistical problems. However, as we have argued in the example from the previous section, distinguishing needs and non-needs variables in (8) ultimately boils down to the normative judgment that differences in health care, due to differences in needs, are ethically legitimate. In the same way demographic standardization in the health case

\footnotetext{
${ }^{8}$ Gravelle (2003) argued that the traditional method of indirect standardization suffers from omitted variables bias, since it only includes the standardizing variables in (8). This problem is easily solved, however, by including all the explanatory variables and following the procedure described in the text (see also Schokkaert and Van de Voorde 2004, 2009, and Fleurbaey and Schokkaert 2009). This procedure is also followed in recent applied work on socioeconomic inequalities in health and health care.
} 
implies that socioeconomic differences in health due to differences in demographics are not inequitable. This suggests that one should broaden the approach and ask for each factor in (8) whether it leads to legitimate or illegitimate differences. As a matter of fact, this immediately raises the question why we should then keep focusing on socioeconomic inequalities only. We will continue that discussion in the next section, where we will propose to start from recent developments in the social choice theory on fair allocation and equality of opportunity. We will then also return to some of the empirical applications that have been mentioned in this subsection.

\subsection{Racial disparities in health care}

While the literature on socioeconomic inequalities is dominated by European authors, in the US there has been much interest for the measurement of racial disparities (inequities) in health care. This is again a partial approach, focusing only on one possible dimension of inequity. The concentration index method is obviously not applicable, since race is a categorical variable without a natural order. Still, as we will see, the questions that have to be answered when measuring racial disparities are related to the issues raised in the previous section. We will first discuss the principles and then briefly describe some empirical applications.

As the literature on racial disparities focuses on health care, we will do the same in this section. A similar approach could be developed to measure disparities in health, however. Moreover, the methods described in this section can also be applied for measuring other disparities linked to a discrete variable, e.g., gender.

\subsubsection{Principles}

For simplicity, focus on the case of two groups, Black and White. An immediate indicator of inequality in the use of health care would be $\mu^{W}(h c)-\mu^{B}(h c)$, which is simply the difference in mean health care expenditures for Whites and Blacks. However, for the reasons described before, this difference does not necessarily point to any inequity, e.g., if there are differences in needs between Whites and Blacks. Obviously, a needs correction is necessary before we can talk about an inequitable "disparity". Yet, as illustrated by Table 1, health service utilization is determined by many other variables apart from race and needs. The partial nature of the approach raises the question of how these other variables have to be treated. 
Let us distinguish four sets of variables: race $\left(R_{i}\right.$, a dummy variable taking the value 1 for Whites), health care needs $\left(h n_{i}\right)$, socioeconomic status $\left(S E S_{i}\right)$ and preferences $\left(P_{i}\right)$. The latter three can be vectors of indicators. Socioeconomic status variables may include income, education, region, and so on. Health care needs can be approximated by direct measures of health and by demographic variables such as age and sex. The basic issue of how to measure inequitable disparity can then be most easily illustrated for the case in which an additively separable function describes the link between these sets of variables $^{9}$ :

$$
h c_{i}=\alpha+\beta R_{i}+\gamma \psi\left(S E S_{i}\right)+\delta \chi\left(h n_{i}\right)+\theta \nu\left(P_{i}\right)+\varepsilon_{i},
$$

where $\varepsilon_{i}$ is a disturbance term.

A natural measure of racial disparity in health care seems to be the estimated parameter $\widehat{\beta}$, i.e., the average difference in health care between Whites and Blacks after having corrected for all the variables in (12). However, restricting ourselves to $\widehat{\beta}$ means that we do not only correct for the differences in needs $h n_{i}$, but also for the differences in socioeconomic status. This is a debatable approach. Suppose (realistically) that Blacks are overrepresented in lower SES groups and that SES also leads to differences in health care (even after correcting for needs): should this indirect effect through SES not be taken into account in measuring overall racial disparity?

The US Institute of Medicine (IOM) defines disparities in health care as “...racial or ethnic differences in the quality of health care that are not due to access-related factors or clinical needs, preferences and appropriateness of intervention" (Institute of Medicine 2002, p. 32). This definition gives an explicit list of "legitimate" reasons for differences (needs and preferences), implying that the contribution of other factors (such as SES) to health care differences is illegitimate and should be included when measuring disparity. Implementing this definition requires constructing a counterfactual situation in which Blacks and Whites have the same health status (and preferences) but differ in SES. This yields two possible measures of disparity. The first takes the Black consumption of health care as reference and computes

$$
\text { dis }=E\left(h c_{i} \mid R=1, S E S=W, h n=B, P=B\right)-E\left(h c_{i} \mid B\right)
$$

where $E\left(h c_{i} \mid R=1, S E S=W, h n=B, P=B\right)$ is a shorthand expression for average health care in the counterfactual situation of White race, the White distribution of SES, the Black distribution of health

\footnotetext{
${ }^{9}$ This is a slight extension of the linear eqs. (8) and (11), that were introduced in our discussion of the CI-approach. In this case also, eq. (12) is to be interpreted as a reduced form.
} 
care needs and the Black distribution of preferences. The expression $E\left(h c_{i} \mid B\right)$ stands for average health care for the Black. The second measure, taking the White consumption as the reference, yields

$$
d i s^{*}=E\left(h c_{i} \mid W\right)-E\left(h c_{i} \mid R=0, S E S=B, h n=W, P=W\right)
$$

There are no obvious theoretical reasons to choose between these two definitions, although one might claim that the former is perhaps a bit more politically relevant as it shows the difference between the Black health care consumption and their hypothetical consumption if there were no inequity. The distinction does not matter in the additively separable case, for which the two definitions coincide. In this case, racial disparity can be computed easily as it only requires information about the means of the distributions of the explanatory variables:

$$
d i s=d i s^{*}=\widehat{\beta}+\widehat{\gamma}\left(E\left(\psi\left(S E S_{i}\right) \mid W\right)-E\left(\psi\left(S E S_{i}\right) \mid B\right)\right)
$$

The interpretation is straightforward. Racial disparity is the sum of the "direct" effect of race $\widehat{\beta}$ and the indirect effect that works through the difference in average SES (multiplied by the effect $\widehat{\gamma}$ of SES on health care). This approach can easily be extended to the more general case where the coefficients in (12) are different for Blacks and Whites:

$$
h c_{i}=\alpha+\beta R_{i}+\left(\gamma+\mu R_{i}\right) \psi\left(S E S_{i}\right)+\left(\delta+\eta R_{i}\right) \chi\left(N_{i}\right)+\left(\theta+\tau R_{i}\right) \nu\left(P_{i}\right)+\varepsilon_{i},
$$

for which we get (applying as in (10) the Blinder-Oaxaca decomposition)

$$
\begin{aligned}
\operatorname{dis}=\widehat{\beta}+\widehat{\gamma}\left(E\left(\psi\left(S E S_{i}\right) \mid W\right)-E\left(\psi\left(S E S_{i}\right) \mid B\right)\right) & \\
& +\widehat{\mu} E\left(\psi\left(S E S_{i}\right) \mid W\right)+\hat{\eta} E\left(\chi\left(N_{i}\right) \mid B\right)+\hat{\tau} E\left(\nu\left(P_{i}\right) \mid B\right) .
\end{aligned}
$$

The additional terms capture the fact that socioeconomic status, needs and preferences may have a different effect on health care for the White and for the Black.

The calculation of racial disparities becomes much more tricky, however, as soon as the function linking health care to race, socioeconomic status, needs and preferences is not additively separable. Information about the means of the distributions is then no longer sufficient to compute the relevant counterfactual situation. In fact, there are different possibilities to set up the counterfactual. Moreover, the two approaches (leading to dis and $d i s^{*}$ ) are no longer equivalent. Cook et al. (2009) have proposed two interesting measures for this case. For the ease of exposition, we neglect the effect of preferences and we assume that $S E S$ and $h n$ are unidimensional (i.e., real numbers). The measure $d i s^{*}$ in $(14)^{10}$ can

\footnotetext{
${ }^{10}$ Cook et al. (2009) focus on the other case with black consumption as the reference. We have swapped the role of $B$ and $W$ here, for convenience.
} 
then be written as

$$
E\left(h c_{i} \mid W\right)-\iint h c(B, S E S, h n) f^{*}(S E S, h n) d S E S d h n
$$

with a fictitious distribution $f^{*}$ that has the same marginal for $S E S$ as the Black and the same marginal for $h n$ as the White. Different methods of calculating disparities boil down to different specifications of $f^{*}$.

One method, the "rank and replace" method adjusts health status (needs) by ranking both the sample of Blacks and that of Whites by the values of $h n$ and then replacing the value of $h n$ of each Black individual with that of the correspondingly ranked White individual. Formally this means that one formulates $h n$ as a function of race and percentile, $\eta\left(R_{i}, \pi_{i}\right)$, where $\pi_{i}$ is the percentile of the individual in the distribution of $h n$ for his racial group. In order to calculate $d i s^{*}$ one then replaces $h n_{i}$ for every Black by $\eta\left(W, \pi_{i}\right)$. Disparity can then be computed as

$$
E\left(h c_{i} \mid W\right)-\iint h c(B, S E S, \eta(W, \pi)) f(S E S, \pi \mid B) d S E S d \pi
$$

which is equivalent to (17) with

$$
f^{*}(S E S, h n)=f(S E S, F(h n \mid W) \mid B) f(h n \mid W)
$$

where $F(h n \mid W)$ is the conditional CDF of $h n$, i.e., is the solution $\pi$ to the equation $\eta(W, \pi)=h n$.

The other method proposed in Cook et al. (2009) combines a rank and replace method with a correction by propensity scores. The first step is the construction with propensity scores of the following artificial distribution:

$$
f(S E S, h n \mid W) \frac{f(S E S \mid B)}{f(S E S \mid W)}=f(h n \mid S E S, W) f(S E S \mid B),
$$

which automatically gives the marginal distribution of the Black for SES. However, this first correction is not sufficient as it does not yield the White marginal distribution of $h n$. Therefore, in a second step the rank and replace method is applied, and one then computes disparity as

$$
E\left(h c_{i} \mid W\right)-\int h c\left(B, S E S, \eta\left(W, F_{0}(h n)\right)\right) f(h n \mid S E S, W) f(S E S \mid B) d S E S d h n
$$

where $F_{0}(h n)=\int F(h n \mid S E S, W) f(S E S \mid B) d S E S$.

It is not obvious how to choose between these two approaches. We will see in the next subsection that they may give similar results when applied to empirical data but this is not more than a convenient answer. We discuss some deeper normative questions in the following section. 
At a more general level, the partial approach to measuring racial disparities raises similar questions as the ones we formulated already with respect to the use of the concentration index. First, why only focus on racial disparities? If racial differences mediated by socioeconomic differences are ethically illegitimate, then surely the same must be true for socioeconomic differences in health care use themselves, again of course after correcting for differences in needs. In this respect, Kawachi et al. (2005) argue that "race" and "class" should be seen as separate constructs and that an adequate measurement of inequity should explicitly analyze their independent and interactive effects. They emphasize that making race a highly visible feature of economic policy has the consequence (in their political analysis even the goal) of hiding or disguising class differences. As will become clear later on, we agree that one should take a broad view of (in)equity, integrating different dimensions. Even in such a broad approach, however, it can make sense to focus on the specific effects of one indicator, e.g., to focus on racial disparities. The question then remains how to integrate such a partial indicator in the overall picture. A priori, it is not impossible that policy measures lowering racial disparities lead to an increase of the overall inequity of the system.

Second, and related to the first question: why concentrate on the differences in the means? If one is exclusively interested in the overall health care disparity between two well-defined groups such a focus on the difference in the means seems natural. However, in a broader perspective, it can hide substantial differences in the degree of inequitable treatment within each of the groups. The same average difference can follow from an almost uniform difference between Blacks and Whites in all regions of a country and across socioeconomic positions, but it may also be the case that some groups of Blacks are treated reasonably well, while for other groups the situation is extremely bad. It seems worthwhile to develop a richer approach that is able to accommodate such within-group inequalities.

\subsubsection{Empirical applications}

The overall message of the extensive literature on racial and other health disparities in the US is clear. A detailed overview of the results, including an extensive list of references, can be found in the yearly National Healthcare Disparities Report (see, e.g., Agency for Healthcare Research and Quality 2009). Racial disparities do exist and they are to the disadvantage of minority groups such as the Blacks and the Latinos. Rather than giving a necessarily incomplete overview of that literature, we will focus as before on some important methodological developments and questions.

First, it is essential to see if the different methods presented in the previous subsection indeed lead to different results. Some representative results from Cook et al. (2009) for Black-White disparities 
in total medical expenditures (2003-2004) and from Cook et al. (2010) for Black-White disparities in mental health care (2002-2006) are summarized in Table 3. It is clear that the measured disparity does not simply equal the unadjusted difference and that the adjustment method does matter. Comparing the results for the first two rows shows that the unadjusted difference in total medical expenditures is larger than the coefficient of race in a linear model: this is because there is a negative correlation between SES and total medical expenditures. The opposite is true for mental health expenditures. Including the indirect effect of SES through either the rank-and-replace method or the refined propensity score method has a strong effect on measured disparities. In all cases racial disparities as defined before, i.e., including the indirect effect of SES, are larger than the unadjusted difference.

Second, the empirical results show how important it is to go beyond simple linear models for the explanation of health care expenditures. This does not only have an effect on the measured disparity (compare the two last rows in Table 3 with the other rows), it also gives a richer picture of reality and makes it possible to derive more convincing policy conclusions. As an example, Cook et al. (2010) use a two-part generalized linear model to explain mental health expenditures and find that there are important Black-White disparities in reaching the first mental health visit and filling the first prescription, while there are no Black-White disparities among those using mental health care services. ${ }^{11}$ This points out that discrimination is probably less important than barriers to entry - and suggests that policy measures should focus on the latter. Such differentiated conclusions can only be obtained when one estimates a rich explanatory model, which also means of course that one needs disparity measures that can be used with such a non-linear model. Remember that for exactly the same reason we have been critical in section 3.1.3 about the use of linear decompositions in the concentration index approach.

Third, as soon as we want to adjust differences in health care expenditures for differences in needs and preferences and we want to include at the same time the indirect effect of socioeconomic status, it is evident that the results will depend on the quality of the information on needs, preferences and socioeconomic status. Cook et al. (2009) have a rich dataset with detailed information on medical conditions and on different dimensions of socioeconomic status (including education, income, region, the fact of being insured) and they test how robust their conclusions are when some of this information is not used. Perhaps not surprisingly, it turns out that the omission of the medical conditions has a strong (negative) effect on the calculation of racial disparities, while the use of imperfect information on socioeconomic status is less harmful. Demographic variables (such as age and gender) and self reported health are definitely not

\footnotetext{
${ }^{11}$ The results for the Latinos are different - see Cook et al. (2010).
} 
sufficient for an adequate needs correction. Note, moreover, that using demographic variables like age and gender as needs indicators, makes it difficult to analyse gender or age disparities. If a given gender or age group is discriminated against by the health care system, it will be impossible to identify this effect in a reduced model, as both this potential discrimination and the needs differences will be captured by the same coefficients.

Table 3. Black-White disparities in health care and mental health care

\begin{tabular}{|l|c|c|}
\cline { 2 - 3 } \multicolumn{1}{c|}{} & $\begin{array}{c}\text { Total medical expenditures } \\
(2003-2004)\end{array}$ & $\begin{array}{c}\text { Mental health expenditures } \\
(2002-2006)\end{array}$ \\
\hline Unadjusted difference & $\$ 1082$ & $\$ 57$ \\
\hline Full adjustment (coefficient in linear model) & $\$ 489$ & na \\
\hline Oaxaca-Blinder decomposition & $\$ 913$ & $\$ 83$ \\
\hline Rank and replace & $\$ 1407$ & $\$ 111$ \\
\hline Propensity score with rank and replace & $\$ 1454$ & \multirow{2}{*}{} \\
\hline
\end{tabular}

Source: Cook et al. (2009), Cook et al. (2010)

Fourth, the missing variable in almost all of the empirical work is preferences. As mentioned before, in the definition of health care disparities by the US Institute of Medicine, preferences are explicitly mentioned as a legitimate reason for health care differences. In empirical work, however, such preference differences are impossible to identify if one does not have direct preference indicators. In general the effect of the variable "race" will therefore not only represent direct illegitimate health care disparities, but will at the same time also capture preference differences. How important are these? Ayanian et al. (1999) conducted personal interviews to collect direct information on preferences with respect to renal transplantation. They report that Black patients were less likely than White patients to want a transplant. However, these preference differences explain only a small fraction of the racial differences in access to transplantation. Other authors have raised a series of critical questions with respect to the interpretation of what might look like preference differences (Ashton et al. 2003, Armstrong et al. 2006). They point out that different attitudes can also reflect differences in communication skills and a possible lack of concordance of the communication style of the provider with the expectations of the patients, can reflect differences in trust in the system, or the lack of adaptation of supply side characteristics to cultural differences - some cultural preferences (those of the White middle class) are better accommodated than 
others, because there is a greater willingness to accommodate them. In all these cases, what seem to be preference differences are ultimately shaped by the practices of the health care system itself. The treatment of preferences raises tricky issues, which are also important from a philosophical point of view. We will discuss them further in section 4.2 .

\section{A more general approach? Equality of opportunity in health and health care}

In the previous section we have reviewed partial measures of inequity focusing on the influence of one specific individual or group characteristic (income, socioeconomic status, race) on health or on health care. We have raised the point that it is important — but not straightforward — to integrate these partial approaches in a broader view on the measurement of inequity. This could also help to strengthen the links between the philosophical debate on equity and the formal approaches in the applied health economic literature. In this section we propose to relate these issues to similar considerations that have recently emerged in the subfield of normative economics that deals with the concept of equal opportunities. The connection is quite direct because the theory of equal opportunities also revolves around the goal of compensating (or neutralizing) the influence of certain characteristics (such as social background or innate talent) on a relevant outcome, while disregarding the inequalities generated by other variables (such as effort or preferences). The core of the theory is about the structure of this partial compensation problem and can be applied to many different contexts, depending on how the boundary is drawn between the variables to be compensated and the other variables. The insights obtained in this field may therefore be helpful for the study of inequalities in health and health care (see, e.g., the editorial of Rosa Dias and Jones 2007). Indeed, the number of empirical applications of the framework is now rapidly growing, both inside and outside the health context.

We first introduce the theory of equal opportunities and examine its application to health and health care, making connections with the partial approaches described in the previous section. In the second subsection we discuss the problem of where to draw the line between "legitimate" and "illegitimate" reasons for differences in health and health care, i.e., between variables for which no compensation is needed and variables for which compensation is necessary. We illustrate this somewhat philosophical discussion for the specific case of lifestyle differences, which will confront us again with the problem of 
how to interpret preferences. Finally, we give an overview of the most important empirical applications of the approach in the domain of health and health care.

\subsection{Equality of opportunity and fairness in health and health care}

In normative economics, the idea of equalizing opportunities has generated a literature on how to deal with the distinction between legitimate and illegitimate inequalities. ${ }^{12}$ Assume that among the different factors leading to inequalities among individuals, some do not lead to illegitimate inequalities, while the others do. The usual motivation for such a partitioning of factors, in the context of equalizing opportunities, relates to personal responsibility. For instance, the individual's social background will typically be considered a source of illegitimate inequality, while the individual's effort may be considered a source of legitimate inequality. Throughout this subsection we will ignore the philosophical debate about the partition, however, and we will focus on the formal structure of the compensation problem. The broader debate will be taken up in the second subsection.

It is useful to set the stage with the description of the approach proposed by Roemer $(1993,1998$, 2002), the most popular in applied work in health economics. Suppose that a certain variable of individual success or advantage, denoted $y_{i}$, is determined by a vector of illegitimate factors of inequality called "circumstances" and denoted $c_{i}$ and a vector of legitimate factors called "effort" and denoted $e_{i}$, via some function $y$. This function does not only incorporate the technology of production of $y_{i}$ but also the prevailing institutions that organize redistribution across individuals with different characteristics:

$$
y_{i}=y\left(c_{i}, e_{i}\right)
$$

In Roemer's terminology, individuals with the same circumstances are defined as being of the same type. Roemer then claims that equality of opportunity requires that individuals at the same level of effort should attain the same level of advantage. Other things equal, at any given effort level $\widetilde{e}$, the social objective could then be to maximize the advantage of the individual that is worst-off, i.e., that has the lowest level of advantage ${ }^{13}$ :

$$
\max \min _{c} y(c, \widetilde{e})
$$

\footnotetext{
${ }^{12}$ A survey of the literature has been made by Fleurbaey and Maniquet (2011) and comprehensive monographs have been written by Roemer (1998) and by Fleurbaey (2008).

${ }^{13}$ This maximin-criterion is the natural extension of egalitarianism if one is also concerned about efficiency. Indeed, it implies that inequalities are only acceptable if they are to the advantage of the worst-off in society.
} 
This criterion is not complete, as one still has to aggregate over the different effort levels. Given that differences due to effort are not illegitimate from an ethical point of view, Roemer suggests that a natural aggregation method is to take simply the sum (or the mean) over the outcomes at different effort levels. This yields the following social objective:

$$
\max \int_{e} \min _{c} y(c, e) f(e) d e
$$

where $f(e)$ is the density function of effort in society.

Until now we assumed that "effort" can be measured and compared between types unambiguously. This is not always evident, however. Already in an early stage of the development of his theory, Roemer (1993) used a health example to illustrate the problem. Suppose the outcome is health, there are two types (blue and white-collar workers) and effort is given by the number of cigarettes smoked. If bluecollar workers as a group smoke more on average, it is debatable to hold individual blue-collar workers responsible for their higher level of smoking, which is ultimately a characteristic of their type. Roemer therefore proposes to define effort directly as the rank (or percentile) of individual $i$ in the distribution of cigarettes smoked for his own type. If there is a monotonic relationship between health and number of cigarettes smoked, the rank of individual $i$ in the distribution for his type of cigarettes smoked will be the same as his rank in the distribution of health for his type. We can then rewrite (20) as

$$
\max \int_{\pi} \min _{c} y\left(c_{i}, \pi\right) d \pi
$$

where $\pi_{i}$ is the percentile of the individual $i$ in the conditional distribution of outcome for his type $F(y \mid c)$.

While the Roemer approach has become popular in applied work, it is only one among several possible approaches. It makes specific assumptions that may not always be attractive, and the literature contains a number of interesting alternatives. We will therefore first introduce a more general formal framework for measuring the unfairness due to illegitimate inequalities. In this more general framework, the Roemer approach will be one special case. We will then show how the theory of selective (or responsibilitysensitive) egalitarianism can be applied to measuring inequity in health and health care. Finally we discuss how the concentration index and the measurement of racial disparities can be reinterpreted within this framework. 


\subsubsection{The formal framework of selective egalitarianism}

In order to link our discussion more closely to the existing literature, let us briefly introduce the general concepts before examining how they can be applied to health. ${ }^{14}$ A natural starting point is to depart from the measurement of inequalities in "total advantage" $y_{i}$ and to construct a "partial" measure of the advantage of individual $i$ in a way consistent with the idea that what is due to $e_{i}$ does not matter for measuring inequalities and that only differences in $c_{i}$ lead to illegitimate inequalities. An obvious solution consists in focusing on the expected value of the outcome given a certain value of $c_{i}$, i.e., $E\left(y \mid c_{i}\right)$. For individual $i$, the value of $E\left(y \mid c_{i}\right)$ can be considered as a measure of the part of his personal advantage $y_{i}$ that is linked to $c_{i}$. One can then apply any suitable inequality index to the distribution of $E\left(y \mid c_{i}\right)$ over the population. Let us call this the "inequality of means" approach. ${ }^{15}$

This natural solution has a substantial shortcoming, however. Remember that the basic social objective in this approach is the elimination of illegitimate inequalities in individual advantage $y_{i}$, as determined by (19), where illegitimate inequalities are those linked to $c_{i}$. The goal of eliminating such inequalities is captured by the "compensation principle", according to which between individuals with identical $e_{i}$, priority should be given to the worse-off..$^{16}$ Equality of $E\left(y \mid c_{i}\right)$ across individuals (or, equivalently, across types formed by different values of $c_{i}$ ) is compatible with arbitrarily large inequalities between individuals having the same $e$ characteristics. The "inequality of means" approach therefore does not satisfy the compensation principle. There is an easy graphical way to see this. When all individuals have equal access to the same range of values of $e_{i}$, and if $c_{i}$ is a fixed characteristic of individual $i$, one may consider that the whole graph of $y\left(c_{i}, \cdot\right)$ represents the opportunities offered to individual $i$. Equalizing $E\left(y \mid c_{i}\right)$ then corresponds to equalizing the areas below the graphs $y\left(c_{i}, \cdot\right)$, as in Figure 2a, while the full elimination of illegitimate inequalities would require that all the graphs of $y\left(c_{i}, \cdot\right)$ should be equal, as in Figure 2b.

A remedy to this problem is the following. For every individual $i$, consider the subpopulation of individuals having the same $e_{i}$ as $i$, and compute the equally-distributed equivalent of the distribution of

\footnotetext{
${ }^{14}$ In the paradigmatic case from the literature, $y$ could refer to income (or welfare), $c$ would be natural talent or socioeconomic status, $e$ could stand for effort.

${ }^{15}$ Some authors (e.g., Lefranc et al. 2009) have proposed to analyse the degree of inequality of opportunity in terms of more general characteristics of the conditional distributions $F\left(y \mid c_{i}\right)$. We will return to this approach in our discussion of empirical applications (section 4.3). In this section we focus on the derivation of specific inequality measures.

${ }^{16}$ In Roemer's approach, absolute priority is given to the worst-off, but less extreme degrees of priority can be considered.
} 


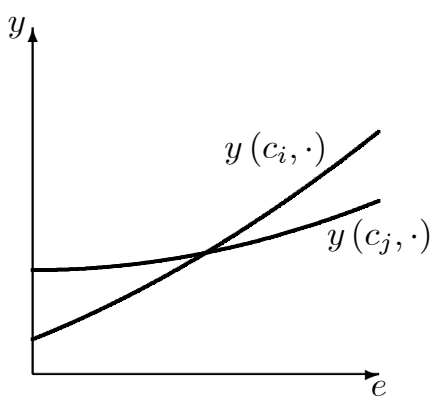

(a)

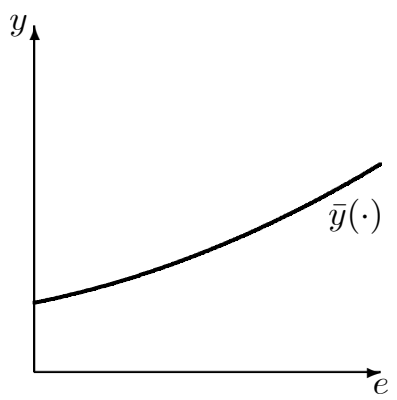

(b)

Figure 2: Equalizing opportunities

$y_{i}$ in every subgroup formed by individuals with the same $e_{i}: E D E\left(y \mid e_{i}\right) .{ }^{17}$ The value of $E D E\left(y \mid e_{i}\right)$ does not measure the partial advantage that $i$ gains from his own $c_{i}$, but it measures what $y_{i}$ would be if the illegitimate inequalities in the subgroup sharing $e_{i}$ were eliminated. Taking the average (or the sum) of these quantities for the different values of $e_{i}$ yields a sensible social welfare objective. Let us call this the "average EDE" approach. The Roemer criterion, as defined in (20), is one member of this family with an inequality aversion equal to infinity, such that $E D E\left(y \mid e_{i}\right)=\min _{c} y\left(c, e_{i}\right)$.

Although this approach does not deliver an individual measure of partial advantage, an interesting index of illegitimate inequalities can be derived by computing the fraction (for a relative index) or the per capita amount (for an absolute index) of total $y$ over the population that would be lost (or, stated alternatively, could be "saved") if every individual's $y_{i}$ were replaced by $E D E\left(y \mid e_{i}\right)$. Such an index is equal to zero only if $y_{i}$ is equal to $E D E\left(y \mid e_{i}\right)$ for every $i$, which is obtained only when $y$ is equalized within every subpopulation sharing the same value of $e$, as requested by the compensation principle. There is a whole family of such measures, with each member of the family corresponding to a specific way of measuring $E D E\left(y \mid e_{i}\right)$, and in particular a specific degree of inequality aversion. ${ }^{18}$

The measures we have considered until now have one important, questionable feature in common. To see this, consider the special case in which there is only one value of $c$ in the population, so that there is no illegitimate source of inequality. Everyone then shares the same $E\left(y \mid c_{i}\right)$, and $E D E\left(y \mid e_{i}\right)$ coincides

\footnotetext{
${ }^{17}$ Recall that the equally-distributed equivalent is the value of individual outcome such that a perfectly equal distribution in which everyone enjoys this value of outcome is considered as equally good from the point of view of social welfare as the contemplated distribution. Under strict inequality aversion, the equally-distributed equivalent is strictly below the mean of the distribution whenever there are inequalities.

${ }^{18}$ It is also obviously possible to simply compute an inequality index for every subgroup sharing the same value of $e$, and then add up the indices over the whole population. Such an approach, however, is harder to relate to a sensible notion of social welfare.
} 
with $y_{i}$ for every $i$, so that the average value of $E D E\left(y \mid e_{i}\right)$ over the individuals is the same as $E\left(y \mid c_{i}\right)$. In other words, the two approaches introduced here (inequality of means and average EDE) merge and advocate focusing on $E\left(y \mid c_{i}\right)$. This means that in this particular context, social welfare boils down to the sum or the average of $y_{i}$ over the whole population. At first sight this is not unreasonable. After all, maximizing the sum or average is tantamount to applying a social criterion with no aversion to inequality, which seems well in line with the idea that inequalities do not matter when they come from $e$. This was also the intuition behind Roemer's social objective function in (20). However, most theories of equality of opportunities advocate a different sort of policy for this special situation in which there is only one value of $c$ in the population. They recommend a laisser-faire policy in which no particular transfers are made across individuals having different values of $e$. Indeed, what reason would there be to intervene if there is no illegitimate source of inequality? If maximizing the sum or average of $y$ over the population required massive redistribution in favor of individuals whose value of $e$ increases their marginal productivity of $y$ with respect to resources, there would be a serious clash with this liberal recommendation of no transfer.

This discussion provides an important insight. The compensation principle is fully satisfied when the graphs of $y\left(c_{i}, \cdot\right)$ are equalized across individuals (or types), but is silent about the shape of the graph $y\left(c_{i}, \cdot\right)$. It is therefore possible to satisfy the compensation principle in the so-called "utilitarian" way that seeks to maximize the area below the graph of $y\left(c_{i}, \cdot\right)$, or in the "liberal" way that seeks to make $y\left(c_{i}, \cdot\right)$ correspond to the laisser-faire policy, or in many other ways. The theory of equality of opportunity is not complete until the compensation principle is supplemented by a reward principle that tells how advantage should be apportioned to effort, i.e., that specifies the desirable shape of the graph $y\left(c_{i}, \cdot\right)$. This also bears on the idea of measuring advantage in a partial way so as to focus on what is due to $c_{i}$. Such a measure necessarily reflects a particular reward principle, and one must therefore be careful to choose a measure that is consistent with the reward principle one believes in. As the reward principle underlying a particular measure is often implicit and hidden in the structure of the measure, it is a necessary and important preliminary step to check what it is before adopting a measure. This can be done, as in the previous paragraphs, by looking at what happens in absence of inequalities in $c$.

Before turning to the measures that are in line with the liberal reward principle, let us introduce another important observation made by the theory of equal opportunity. The compensation principle and the reward principle, whatever the latter is, tend to clash in the sense that a measure that satisfies the compensation principle is typically less satisfactory with respect to the reward principle, and vice versa. This point can be illustrated with the two measures introduced before. When one measures inequalities 
in $E\left(y \mid c_{i}\right)$, one is totally indifferent to modifications of the distribution of $y$ in a subgroup of individuals with the same $c$, provided that the sum of $y$ remains unchanged in this subgroup. This very well reflects the "utilitarian" reward principle stipulating that for individuals with the same c, one should have no inequality aversion and be interested only in the sum or the mean. However, as emphasized before, this "inequality of means" approach does not satisfy the compensation principle. In contrast, the measure that is based on $E D E\left(y \mid e_{i}\right)$ can be affected by such redistributions of $y$ within a subgroup of individuals with the same $c$, because the values of $\operatorname{EDE}\left(y \mid e_{i}\right)$ for various values of $e_{i}$ will then typically be altered. This second measure is indifferent to such redistributions only when all individuals share the same $c$. The "average EDE" measures, satisfying the compensation principle, are therefore less strict on the utilitarian reward principle.

This tension between compensation and reward can be illustrated also by measures that are based on the liberal variant of the reward principle. Such a "liberal" view about reward requires a richer framework in which the transfers to which individuals are submitted are explicitly distinguished in the outcome function. Letting $t_{i}$ denote the transfer to which individual $i$ is submitted, (19) is refined into:

$$
y_{i}=y\left(t_{i}, c_{i}, e_{i}\right) .
$$

This new function $y$ only reflects the technology of production of $y_{i}$, and no longer the transfer policy which is an explicit argument of it. The liberal reward principle then stipulates that, among individuals with the same $c_{i}$, priority should be given to those with a lower $t_{i}$ (independently of their $e_{i}$ ). This implies that the transfers are ideally null in the hypothetical case in which there is only one value of $c$.

Again, in this new framework there is a natural solution, which consists in fixing a reference value for $e_{i}, \tilde{e}$, and considering that illegitimate inequalities are those that arise for the values of $y\left(t_{i}, c_{i}, \tilde{e}\right)$. One can then simply focus on the distribution of these values instead of the distribution of $y_{i}$. Each individual $i$ is treated as if $e_{i}=\tilde{e}$, ignoring the part of $y_{i}$ that comes from a discrepancy between $e_{i}$ and $\tilde{e}$. This approach is called "conditional equality" in the literature, in reference to the fact that it seeks equality conditionally on individuals adopting $\tilde{e}$. Note that equalizing the values of $y\left(t_{i}, c_{i}, \tilde{e}\right)$ across individuals with identical $c$ implies giving them equivalent $t$, as requested by the liberal principle of reward. But this approach fails the compensation principle whenever it may happen that there are two individuals $i$ and $j$ with identical $e_{i}$ and such that $y_{i}<y_{j}$, while $y\left(t_{i}, c_{i}, \tilde{e}\right)>y\left(t_{j}, c_{j}, \tilde{e}\right) \cdot{ }^{19}$ Compensation is guaranteed

\footnotetext{
${ }^{19}$ Note that conditional equality does satisfy the compensation principle if the function $y($.$) takes a specific form, e.g., if$ it is separable such that $y_{i}=f\left(g\left(t_{i}, c_{i}\right), e_{i}\right)$. The worse off in $f\left(g\left(t_{i}, c_{i}\right), \tilde{e}\right)$ are the worse off in $g\left(t_{i}, c_{i}\right)$, and therefore the
} 
only for those with $e_{i}=\tilde{e}$.

The compensation principle is satisfied by the dual approach which fixes a reference value for $c_{i}, \tilde{c}$, and focuses on the inequalities in $t_{i}^{*}$ across individuals, where $t_{i}^{*}$ is the solution to the equation

$$
y_{i}=y\left(t_{i}^{*}, \tilde{c}, e_{i}\right) .
$$

An intuitive understanding of this approach can be obtained by looking at what happens when equality of $t_{i}^{*}$ across individuals is achieved. One then has a situation that is equivalent to a situation in which differences in $y_{i}$ are only due to $e_{i}$, because $y_{i}=y\left(t^{*}, \tilde{c}, e_{i}\right)$ for a given $t^{*}$. These inequalities are thus legitimate. This approach is therefore called "egalitarian-equivalence" because it seeks equivalence with an egalitarian situation. The cost of satisfying the compensation principle is that it may happen that inequalities in $t_{i}^{*}$ may sometimes be the opposite of inequalities in $t_{i}$ for some individuals with identical $c_{i}$, implying a failure of the liberal principle of reward for such individuals. Liberal reward is guaranteed only for those with $c_{i}=\tilde{c}^{20}$

Table 4. Variants of responsibility-sensitive egalitarianism

\begin{tabular}{|l|l|l|}
\cline { 2 - 3 } \multicolumn{1}{c|}{} & Utilitarian & Liberal \\
\hline \hline Compensation principle satisfied & Average $\mathrm{EDE}^{21}$ & Egalitarian-equivalence \\
& $E D E\left(y \mid e_{i}\right)$ & $t_{i}^{*}$ s.t. $y_{i}=y\left(t_{i}^{*}, \widetilde{c}, e_{i}\right)$ \\
\hline Reward principle satisfied & Inequality of means & Conditional equality \\
& $E\left(y \mid c_{i}\right)$ & $y\left(t_{i}, c_{i}, \widetilde{e}\right)$ \\
\hline
\end{tabular}

In conclusion, within the literature on equality of opportunity a number of approaches have been put forward that aim at defining a partial measure of the advantage of individual $i$. "Illegitimate" inequalities can then be measured as inequality in these partial indicators. An overview is given in Table 4. Two insights have been highlighted. First, in addition to the compensation principle, each of the approaches also takes a stance with respect to the reward issue, i.e., the definition of acceptable inequalities. This cannot be ignored even if one only wants to measure illegitimate inequalities. Second, there is a tension between the compensation and reward principles. This tension and the multiplicity of possible reward principles create a variety of solutions. The four that have been introduced here (see Table 4) provide a

\footnotetext{
worse off in $y_{i}$ for any given $e_{i}$.

${ }^{20}$ Again, the liberal reward principle will be satisfied if $y_{i}=f\left(g\left(t_{i}, c_{i}\right), e_{i}\right)$. Equation $(23)$ is then equivalent to $g\left(t_{i}, c_{i}\right)=$ $g\left(t_{i}^{*}, \tilde{c}\right)$ and for any given $c_{i}$, inequalities in $t_{i}$ are mimicked by inequalities in $t_{i}^{*}$.

${ }^{21}$ In this family of measures, overall inequality is not simply the inequality in the values of $E D E\left(y \mid e_{i}\right)$.
} 
good summary of the main insights. The choice between these different measures will necessarily depend on the attractiveness of the compensation principle and of the different reward principles in the specific case to be analysed.

An important problem is created by configurations in which $c$ and $e$ are not independently distributed and $c$ is suspected of causally influencing $e$, as in Roemer's (1993) smoking example described earlier. In such cases, it seems that for different $c$ the individuals do not have the same access to various values of $e$ and that the graph of $y\left(c_{i}, \cdot\right)$ no longer depicts individual $i$ 's opportunities. A simple theoretical solution to this difficulty is to assume that there is a latent variable of effort $e^{*}$ that is independent of $c$ and such that all individuals have equal access to it. The value of $e$ is a function of $c$ and of this latent variable $e^{*}$. One can thus write $y_{i}$ as a function of $c_{i}$ and $e_{i}^{*}$ and proceed. In the special case in which $y$ is increasing in $e^{*}$, for any given $c$, a rescaling of $e^{*}$ can render the conditional distribution of $e^{*}$ given any $c$ uniform over the interval $[0,1]$. After such rescaling, the graph of $y^{*}\left(c_{i}, \cdot\right)$ corresponds to the inverse of the cumulative distribution function of $y$ given $c$. As we have seen, in a similar vein, Roemer (1993 1998) proposes to measure effort $e_{i}^{*}$ directly as the rank (or percentile) of $i$ in the distribution of $y$ given c. It follows from our discussion that such measurement convention is innocuous if $y$ can be expected to be increasing in $e^{*}$ and if the measure of inequality that is constructed on this basis is not sensitive to rescalings of $e^{*}$. The latter property holds for the four measures introduced here. The former property, however, is ultimately a statement about facts, that does not necessarily hold in all specific situations.

Note that the correlation between $c$ and $e$ does not necessarily imply that the inequalities due to $e$ are partly illegitimate. For theories that ascribe responsibility for preferences, it is natural that preferences are linked to circumstances (bodily characteristics influence the taste for activities depending on such characteristics), but this fact does not detract from the value of respecting preferences and the choices such preferences motivate. It may also happen that $e$ is coincidentally correlated with $c$ without there being a causal impact of $c$ on $e .^{22}$ In such a situation, the "inequality of means" approach is not appropriate because the value of $E\left(y \mid c_{i}\right)$ is influenced by the specific distribution of $e$ conditionally on $c_{i}$. Standardizing the computation of the expected value by taking the same distribution of $e$ for all values of $c_{i}$ is the natural solution to this problem. The "average EDE" approach, in contrast, is not affected because the fact that the computation of $E D E\left(y \mid e_{i}\right)$ involves a different composition of $c$ for different

\footnotetext{
${ }^{22}$ The literature on equal opportunity often presumes causal influence whenever $c$ and $e$ are correlated. Of course, one may interpret a significant correlation between $c$ and $e$ as an indication that they both are linked to the same underlying variables. As we will see within the health context, the ethical implications of this situation may differ from case to case.
} 
values of $e_{i}$ does not matter, the $c$ group to which an individual belongs being essentially an irrelevant piece of information for this approach. Conditional equality and egalitarian-equivalence are also immune to the problem, the former because it ignores $e_{i}$ (except insofar as $t_{i}$ depends on it), the latter because it refers to a hypothetical situation in which everyone has the same $\tilde{c}$.

\subsubsection{Application to health and health care}

The application to health and health care requires a careful reinterpretation of the general framework. Two issues are especially important. First, as was already clear from our discussion of the concentration index and of racial disparities, correlations between the different variables in $e$ and $c$ will be ubiquitous and their interpretation is essential. When $c$ and $e$ are not independently distributed, one must decide whether one is interested in the inequalities generated by $c$ only or in the inequalities generated by $c$ directly and indirectly via its influence on (or correlation with) $e$. Second, a careful reconsideration of the reward principle is needed. Indeed, utilitarian and liberal reward principles are conceived for well-being. In the case of health or health care, there is a special ideal of a good relationship between health care and health needs which does not correspond to either principle and calls for a generalization that we explain below. It will turn out that the compensation principle is strongly linked to the concept of horizontal equity, while different reward principles capture different ideas about vertical equity.

We examine inequalities in health care and in health in turn. As a matter of fact, the intricate link between health care and health induces interesting connections between the two.

Health care The partial approaches to inequity in health care, that have been dominating in the literature until now, focus on the inequalities which are due to the specific influence of a particular socio-demographic characteristic such as gender, race, or socioeconomic status. From a more general perspective one may be interested in all inequalities in health care consumption that are not explained by differences in health needs. In this application the "effort" variables in $e$ are those variables that do not lead to illegitimate inequalities in health care, while inequalities due to circumstances $c$ are ethically objectionable. Therefore, in the partial approaches, the $c$ variable measures the relevant socio-demographic characteristic (e.g., gender or race) and $e$ captures all the rest. In the more general perspective, the $e$ variable measures needs and $c$ is all the rest. There has been a debate about whether differences in preferences and/or information trigger legitimate inequalities in health care, i.e., whether the variable of interest is health care use or access to health care. The various positions in this debate can be accommo- 
dated in the framework discussed here by moving the cut between $c$ and $e$. Once a particular function of health care is written in the form $y(c, e)$, in principle it is possible to apply the different measures that are proposed in the theory. The correlation between $c$ and $e$ is an essential consideration in this context, however, because health needs, which belong to $e$ here, can be expected to be influenced by $c$.

When one constructs a partial indicator (i.e., the inequity due to one $c$ variable, e.g., race), one may be interested not just in the direct effects of that variable but also in the indirect effects via another variable (e.g., socioeconomic status). We have seen that this idea plays an essential role in the definition of racial disparities. In this case, one could in principle write down socioeconomic status as a function of race and other variables, and then distribute the influence of status in the $y$ function between $c$ (race) and $e$ (the rest) accordingly. This decomposition of influences will often be impossible in practice due to data limitations or even deeper limitations in understanding the relevant causal mechanisms. But one can always write a function of statistical dependence, with status $=f($ race, $\pi)$, where $\pi$ is the percentile of the individual in the conditional distribution of status given his race. If status is a discrete variable with few categories, there may remain some arbitrariness in the allocation of particular values of $\pi$ to the different individuals.

To understand the different dimensions of the problem and make them more specific, it is useful to further develop the health care function (which corresponds to the advantage function (22) in this setting):

$$
y\left(t_{i}, c_{i}, e_{i}\right)=h c_{i}=h c\left(h n\left(S E S_{i}, d_{i}, P_{i}^{1}\right), S E S_{i}, d_{i}, P_{i}^{2}\right)
$$

Here $h n$ stands as before for "health needs", determined by a vector $S E S$ of socioeconomic variables (including economic status, race and region), a vector $d$ of demographic variables (such as age and gender) and a vector $P^{1}$ of preference variables influencing lifestyle. Health care is also influenced by the vector $P^{2}$ capturing differences in treatment preferences. Eq. (24) makes it possible to explicitly distinguish two effects: the indirect effect of $S E S$ and $d$ on health care through their effect on health care needs, and the direct effect, pointing to differential treatment (or discrimination) if needs are identical. ${ }^{23}$

Suppose now that we follow the literature on socioeconomic inequalities and take $c=S E S$ and $e=\left(d, P^{1}, P^{2}\right)$. In this interpretation, the compensation principle would impose that health care be equal for individuals with the same value of $e$, i.e., the same demographic and preference variables. This is not attractive, as it would mean that we disregard the effect of $S E S$ on health care needs. A

\footnotetext{
${ }^{23}$ In this sense, eq. (24) is a parsimonious way of writing a simple stylized structural model. Of course, in applied work, one will usually need a more elaborate specification (as in Fleurbaey and Schokkaert 2009).
} 
better approach is to take $c=S E S$ and $e=\left(h n, d, P^{2}\right)$. This obviously implies that $e$, and more specifically $h n$, and $c$ are correlated and therefore rules out the "inequality of means" approach without proper standardization. ${ }^{24}$ For the other measures the correlation does not raise any problem. Focusing on socioeconomic inequalities, i.e., taking $c=S E S$, is only one possibility, however, and other ethical approaches can be accommodated by a different partitioning of the variables in (24) in $c$ and $e$. One could take the position that differences in treatment are legitimate if they reflect differences in needs following from differences in lifestyle preferences $P^{1}$. This would mean that "legitimate" needs have to redefined. Or one could also be concerned about discrimination in health care provision related to $d$ (e.g., age and gender), implying that $c=(S E S, d)$ and $e=\left(h n, P^{2}\right)$. To focus our exposition, we will develop the analysis for the latter case. Our discussion can easily be adapted for the other ethical choices.

Some remarks can be made concerning the interpretation of (24). First, there may be correlation between $c$ and the preference variables: whether this is seen as problematic or not, will depend on the position taken with respect to the boundary between legitimate and illegitimate variables. Second, distinguishing between the direct effect of $S E S$ on health care and its indirect effect through health care needs imposes heavy requirements on the data. Things are even more difficult if we include the demographic variables in $c$, since we most often will need $d$ in the empirical model as a proxy of health care needs.

With $e=\left(h n, P^{2}\right)$, the compensation principle implies that health care should be the same for individuals with the same health care needs and the same treatment preferences. It is immediately clear that this is a direct translation of the principle of horizontal equity. If one follows the dominant approach in the literature and focuses on horizontal inequity, measures that do not satisfy the compensation principle are therefore not very attractive in this setting. What about the reward principles? As described before, the best way of thinking about reward is to consider individuals within the same group of circumstances $c=(S E S, d)$. These individuals may differ in health care needs and treatment preferences: allocating health care to such individuals is immediately related to vertical equity. It is obvious that vertical equity should play a role in evaluating different situations: indeed, horizontal equity would be achieved if all individuals receive the same level of health care regardless of their health care needs (Sutton 2002), and this is clearly not equitable. In fact, it has been emphasized in the literature that it is not possible to

\footnotetext{
${ }^{24}$ Remember that this rejection of the simple "inequality of means" approach was the starting point for the formulation of more complex measures of racial disparity. We will return to this application in the next subsection, but here we will remain at a more general level.
} 
examine the extent to which the horizontal equity principle is violated without simultaneously specifying a vertical equity norm (see, e.g., O'Donnell et al. 2008). This is analogous to the insight from the literature on equality of opportunity that each measure of illegitimate inequality implies the specification of a reward principle. The reward principles from the equality of opportunity literature do not capture well the idea of vertical equity, however. Utilitarian reward focuses on the average amount of health care per group of circumstances $c=(S E S, d)$. Taking a redistribution of health care within such a group as a matter of social indifference is almost like denying the relevance of vertical equity, i.e., of apportioning health care to health needs and preferences. The average amount of health care in a group of circumstances is a poor basis for the evaluation of unfairness at the individual level, even if it might be considered acceptable at the level of comparing circumstance groups themselves. Similarly, liberal reward is problematic because it requires identifying a "transfer" variable contributing to the outcome variable. Health care itself can be viewed as a transfer of resources, but it would make little sense to advocate that everyone should receive the same amount of health care within a group of circumstances. Again, health needs and preferences justify specific amounts of health care.

The fact that health care is itself a policy variable makes it difficult to derive an optimal form of the health care function on the basis of observable empirical evidence. The normatively ideal way in which health care should respond to health needs is linked to the available medical technology and the available resources. Let us posit that within a group of circumstances, there is a given ideal amount of health care, for every member of this group, that depends on health needs and treatment preferences:

$$
h c^{*}\left(h n_{i}, P_{i}^{2} ; S E S_{i}, d_{i}\right) .
$$

It makes sense to consider that this ideal should not depend on socioeconomic status, but it may perhaps depend on demographic variables, e.g., if patients of different ages are legitimately given different degrees of priority (which does not prevent the analyst to seek further differences due to discrimination). We will therefore write it $h c^{*}\left(h n_{i}, P_{i}^{2} ; d_{i}\right)$ from now on. The advantage of individual $i$ can then be defined as the gap between what $i$ receives and the ideal amount:

$$
\Delta_{i}=h c_{i}-h c^{*}\left(h n_{i}, P_{i}^{2} ; d_{i}\right)
$$

and illegitimate inequality can be measured as the inequality in these individual advantage measures.

The difficulty that remains is to define $h c^{*}\left(h n_{i}, P_{i}^{2} ; d_{i}\right)$. Medical information about the best treatment for patients with different needs can be helpful to specify this function, but since $h c^{*}($.$) also has to$ 
capture the availability of resources, such medical information is not sufficient. Some useful approaches can be derived from observable data. Suppose first that $d_{i}$ should actually play no role in the function $h c^{*}\left(h n_{i}, P_{i}^{2} ; d_{i}\right)$. One can then hope that departures from the ideal even out over the population so that one can define the optimal amount of health care as the average quantity of health care received by individuals with the same needs and preferences $e=\left(h n_{i}, P_{i}^{2}\right)$. If one denotes this set of individuals by $N_{e}$ and their number by $n_{e}$, one obtains:

$$
h c^{*}\left(h n_{i}, P_{i}^{2}\right)=h c^{A V}\left(h n_{i}, P_{i}^{2}\right) \equiv \frac{1}{n_{e}} \sum_{i \in N_{e}} h c_{i} .
$$

This is the assumption that is commonly made in the literature on socioeconomic inequity in health care delivery, formulated as: "On average, the system gets it right" (Van Doorslaer et al. 2000 - see also O'Donnell et al. 2008). ${ }^{25}$ When $d_{i}$ does play a role in $h c^{*}\left(h n_{i}, P_{i}^{2} ; d_{i}\right)$, one cannot take the average amount of health care given to a group $\left(h n_{i}, P_{i}^{2}, d_{i}\right)$ as a proxy for the ideal, because discrimination would also influence this average amount. There seems to be no simple statistical way to separate the normal influence of $d_{i}$ on $h c_{i}$ from an abnormal influence linked to discrimination.

Another possibility is to pick a reference value for $c,(\widetilde{S E S}, \widetilde{d})$, and consider that the optimal amount is what individuals with such characteristics receive

$$
h c^{*}\left(h n_{i}, P_{i}^{2}\right)=h c^{R E F}\left(h n_{i}, P_{i}^{2}\right) \equiv h c\left(h n_{i}, \widetilde{S E S}, \widetilde{d}, P_{i}^{2}\right) .
$$

This approach makes much sense if one believes that different socioeconomic groups are treated differently and that the treatment given to one specific group (e.g., the rich or the White) is close to the "optimal" treatment.

Fleurbaey and Schokkaert (2009) have proposed two measures that can be applied to health care as well as to health and are inspired by conditional equality and egalitarian-equivalence (the two liberal criteria introduced in the previous subsection). The measure related to egalitarian-equivalence is the "fairness gap", which in the case of health care and under the assumptions retained here about the $c, e$ classification, is written as

$$
h c_{i}-h c\left(h n_{i}, \widetilde{S E S}, \widetilde{d}, P_{i}^{2}\right)
$$

the general formula being $y_{i}-y\left(\tilde{c}, e_{i}\right)$. This formula corresponds to $(25)$ with the "reference" specification (27). Note that the interpretation in terms of vertical equity suggests an interesting way to think about

\footnotetext{
${ }^{25}$ A refinement to this approach has been proposed by Sutton (2002). We will discuss his approach in the next subsection.
} 
the choice of the reference values $(\widetilde{S E S}, \widetilde{d})$, a choice which is largely left open in the theory of selective egalitarianism.

The similarity with egalitarian-equivalence comes from the presence of a reference $\tilde{c}$ in the formula, and equality of the expressions (28) across individuals implies that individuals with the same $e_{i}=$ $\left(h n_{i}, P_{i}^{2}\right)$ necessarily have the same $y_{i}=h c_{i}$, in conformity with the compensation principle. Inequality in (28) is then an acceptable measure of horizontal inequity. As far as the implications of the reward principle is concerned, imagine a situation in which all individuals share the same $c$ and this value is retained as the reference $\tilde{c}$. Then the fairness gap is null for all individuals, no matter what the $y$ function is. However, if one looks at an ordinary situation with a variety of values of $c$ in the population, and examines how to improve the situation of a particular group sharing the same $c$, one sees that this can be done in a specific way that is not neutral with respect to $e$. To illustrate this point, suppose that $y(c, e)=\alpha+\beta c+\gamma e+\delta c e$, in which all the symbols represent real numbers. One then has

$$
y_{i}-y\left(\tilde{c}, e_{i}\right)=\beta\left(c_{i}-\tilde{c}\right)+\delta\left(c_{i}-\tilde{c}\right) e_{i}
$$

which implies that, in order to reduce inequalities, one should in priority help those with a high $e_{i}$ in subgroups with $c_{i}<\tilde{c}$ (i.e., the unhealthy poor) and those with a low $e_{i}$ in subgroups with $c_{i}>\tilde{c}$ (i.e., the healthy rich). This is easy to understand in the light of the interpretation of $y^{*}(\widetilde{c}, e)=\alpha+\beta \widetilde{c}+\gamma e+\delta \widetilde{c} e$ as the "ideal" health care level corresponding to $e$ (see (27)).

The alternative proposal, related to conditional equality, is simply to evaluate individual advantage as $y\left(c_{i}, \tilde{e}\right)$, which in our example would be $h c\left(\widetilde{h n}, S E S_{i}, d_{i}, \widetilde{P^{2}}\right)$. This measure, called "direct unfairness" by Fleurbaey and Schokkaert (2009), bears a striking similarity with conditional equality but is different because conditional equality is normally computed by retaining the transfer $t_{i}$ fixed, not by computing what the transfer would be with reference $\tilde{e}$. Like conditional equality, it does not satisfy the compensation principle. It is therefore not attractive as a measure of horizontal inequity. In terms of reward, it has the property that the only individuals who are taken into account are those with $e_{i}=\tilde{e}$. Indeed, all the other individuals are in fact ignored because their situation is measured by what the individuals with similar $c$ but reference $e$ would obtain. One must therefore be cautious before using such a measure for normative purposes.

A final observation must be made because it will help connecting the measures described in this subsection with the measures of racial or gender disparity. When reference parameters such as $c$ or $e$ are introduced, one may also think of generalizing by taking a distribution of reference values rather 
than a single value. For instance, an immediate generalization of direct unfairness and the fairness gap can be obtained in this way by computing the expected value of the measures of individual advantage with respect to the distribution of reference parameters. For direct unfairness, if $f(\tilde{e})$ is the reference distribution for $\tilde{e}$, one can compute

$$
\int y\left(c_{i}, \tilde{e}\right) f(\tilde{e}) d \tilde{e}
$$

For the fairness gap, with $g(\tilde{c})$ the reference PDF for $\tilde{c}$, one can compute

$$
y_{i}-\int y\left(\tilde{c}, e_{i}\right) g(\tilde{c}) d \tilde{c}
$$

Note that when the reference distribution of $\tilde{e}$ corresponds to the actual distribution, and when $e$ is independent of $c$, then $\int y\left(c_{i}, \tilde{e}\right) f(\tilde{e}) d \tilde{e}=E\left(y \mid c_{i}\right)$ and direct unfairness coincides with the inequality of means approach. When the reference distribution of $\tilde{c}$ corresponds to the actual distribution, and when $e$ is independent of $c$, the expression $\int y\left(\tilde{c}, e_{i}\right) g(\tilde{c}) d \tilde{c}$ is the average outcome obtained by the group of individuals who have $e_{i}$. Obviously, these generalizations are not innocuous in terms of the implied reward principle.

Health inequalities In the case of health, we can develop the advantage function (22) further along the lines of (24):

$$
y\left(c_{i}, e_{i}\right)=h_{i}=h\left(h c\left(h n\left(S E S_{i}, d_{i}, P_{i}^{1}\right), S E S_{i}, d_{i}, P_{i}^{2}\right), h n\left(S E S_{i}, d_{i}, P_{i}^{1}\right)\right)
$$

According to this function, health is determined by health needs and by health care. ${ }^{26}$ As before, health care depends on health needs. Again, different views on responsibility will be reflected in different ways of allocating the variables at the RHS to either $c$ or $e$. Almost everybody will agree that differences in socioeconomic variables should not lead to differences in health (after controlling for differences in preferences). The large bulk of the literature also takes it for granted that differences in health, caused by the demographic variables $d$, should not be seen as illegitimate, because they apparently cannot be influenced by policy. This position is not beyond criticism, however, as health differences due to demographic variables can be exacerbated or softened through policy (think about rationing decisions in health care) - and one may also take the position that in comparisons of inequity between countries and over time it is useful to include the effects of variables that (in the short run) cannot be influenced by

\footnotetext{
${ }^{26}$ At first sight there may be some confusion between "health" and "health needs". As is made clear by eq. (31), we interpret final health as resulting from health care and (pre health-care) needs.
} 
policy. The most sensitive issues arise with respect to the interpretation of the preference variables. We will come back to that debate in the next section. In this section we will follow the dominant approach and focus on inequalities generated by socioeconomic status. As in the previous subsection, our discussion can easily be adapted for other ethical choices.

In this setting the compensation principle is compelling, and can be expressed by the requirement that "if a measure of unfair inequality is zero, there should be no illegitimate differences left, i.e., two individuals with the same value for the $e$ variable (i.e., the same preferences and the same values for the demographic variables) should have the same health status, i.e., the same outcome $y$." As far as reward is concerned, once again the reward principles have to be reinterpreted carefully because health is not a global index of well-being.

Let us first consider the measures that are based on the utilitarian reward principle. The "inequality of means" approach, that would calculate the inequality in $E\left(h \mid S E S_{i}\right)$ has been advocated by Bommier and Stecklov (2002) for the measurement of socioeconomic health inequalities. As we have seen, this approach is not satisfactory at the bar of compensation and also requires standardization in order to cope with the correlation between $c$ (socioeconomic status) and $e$ (demographic variables). On both counts, as explained above, the average EDE approach is preferable. Both measures rely on the utilitarian reward principle, stating that what matters is average (or total) health for each particular group of circumstances. Maximizing health is a time-honored principle and has become the dominant objective in the literature on cost-effectiveness analysis. Yet, its ethical limitations are by now well understood. Socioeconomic inequalities could be improved, according to such measures, by enforcing health policies for the worstoff socioeconomic groups that give more health care to those whose value of $e$ makes health care more productive with them, thereby increasing average health in these groups. For instance, if the average health among the disadvantaged socioeconomic groups could be increased by sacrificing the severely ill patients and concentrating resources on curable diseases and prevention, this would be seen as improving the situation. These implications of health maximization are hard to swallow and are strongly rejected by the majority of the population (Gaertner and Schokkaert 2011).

As was pointed out in the previous paragraph on health care, the liberal reward principle is not very attractive either, as it seems strange to recommend equal health care for all individuals of the same circumstance group, regardless of their health needs. However, once an ideal health care function $h c^{*}\left(h n_{i}, P_{i}^{2} ; d_{i}\right)$ has been defined, one can extend the theory in order to introduce this benchmark as a 
reward principle. Consider the following measure of individual advantage:

$$
h\left(\Delta_{i}+h c^{*}\left(h n\left(S E S_{i}, \widetilde{d}, \widetilde{P^{1}}\right), \widetilde{P^{2}} ; \widetilde{d}\right), h n\left(S E S_{i}, \widetilde{d}, \widetilde{P^{1}}\right)\right) .
$$

with $\Delta_{i}$ defined as in (25). This leads to a generalization of the conditional equality approach. Indeed, if the ideal $h c^{*}\left(h n_{i}, P_{i}^{2} ; d_{i}\right)$ were constant (within a group of circumstances $S E S$, but as it does not depend on this variable directly it would be constant overall), expression (32) would boil down to

$$
h\left(h c_{i}, h n\left(S E S_{i}, \widetilde{d}, \widetilde{P^{1}}\right)\right),
$$

which is exactly the measure of individual advantage in the traditional conditional equality approach. The generalization (32) instead computes the level of health individual $i$ would enjoy if the irrelevant ("responsibility") characteristics $d_{i}, P_{i}^{1}, P_{i}^{2}$ were at the reference level, and $i$ 's health care was at the corresponding ideal level plus the discrepancy $\Delta_{i}$ that $i$ currently endures with respect to the ideal level $i$ should receive (given $i$ 's actual characteristics). This discrepancy can be interpreted as a specific resource treatment that $i$ is subject to. The idea of generalized conditional equality (32) is to assess how individuals would do if they had the reference irrelevant characteristics and kept $\Delta_{i}$. Keeping $\Delta_{i}$ in the computation makes it possible to assess the consequences of the current unfairness in resources over the outcome (health) for a "reference" situation. When equality of (32) is achieved across individuals of the same SES, they must have the same $\Delta_{i}$.

Alternatively, one can also generalize egalitarian-equivalence. Solve in $t_{i}^{*}$ the equation

$$
h_{i}=h\left(t_{i}^{*}+h c^{*}\left(h n\left(\widetilde{S E S}, d_{i}, P_{i}^{1}\right), P_{i}^{2} ; d_{i}\right), h n\left(\widetilde{S E S}, d_{i}, P_{i}^{1}\right)\right)
$$

Illegitimate inequality can then be measured as inequality in the individual $t_{i}^{*}$. If one could equalize $t_{i}^{*}$ across individuals, one would obtain a situation in which everyone would enjoy a level of health that would be the same as in a hypothetical situation in which health needs are no longer influenced by $S E S$, and in which everyone would receive the ideal amount of health care, up to a uniform discrepancy.

As one can check, generalized egalitarian-equivalence satisfies the compensation principle, whereas generalized conditional equality does not. When two individuals share the same irrelevant characteristics and differ only in their socioeconomic status, their $t_{i}^{*}$ is equal only if their health level is equal. In contrast, if the irrelevant characteristics of these two individuals are not at the reference level, their individual advantages (32) may be equal even if their actual health levels are different. Since the compensation principle is natural in this context, generalized egalitarian equivalence (34) is a more attractive approach than generalized conditional equality. 
However, both approaches make use of an "ideal" health care function $h c^{*}\left(h n_{i}, P_{i}^{2} ; d_{i}\right)$. As discussed before, the specification of this function is an awkward problem, requiring additional assumptions and/or information that is not available in the observable data. As a shortcut, Fleurbaey and Schokkaert (2009) propose what sounds like a weak reward principle. It stipulates that differences in $e_{i}$ (here demographic variables and preferences) across individuals should have as little impact on the measure of illegitimate inequalities as possible. This principle can be reinterpreted if one thinks that the ideal health care function $h c^{*}\left(h n_{i}, P_{i}^{2} ; d_{i}\right)$, which typically depends on $e_{i}$, should be taken as a benchmark in order to measure unfairness at the individual level. As a matter of fact, there are circumstances under which "direct unfairness" and the "fairness gap" coincide with generalized conditional equality and generalized egalitarian equivalence respectively.

Applied to health, direct unfairness measures individual situations as

$$
y\left(c_{i}, \tilde{e}\right)=h\left(h c\left(h n\left(S E S_{i}, \widetilde{d}, \widetilde{P^{1}}\right), S E S_{i}, \widetilde{d}, \widetilde{P^{2}}\right), h n\left(S E S_{i}, \widetilde{d}, \widetilde{P^{1}}\right)\right)
$$

In contrast with the previous measure (32), this one is insensitive to the way in which health care reacts to different values of $e$ characteristics. The direct unfairness measure for individual $i$ then equals her individual advantage with generalized conditional equality if

$$
\Delta_{i} \equiv h c_{i}-h c^{*}\left(h n_{i}, P_{i}^{2} ; d_{i}\right)=h c\left(h n\left(S E S_{i}, \widetilde{d}, \widetilde{P^{1}}\right), S E S_{i}, \widetilde{d}, \widetilde{P^{2}}\right)-h c^{*}\left(h n\left(S E S_{i}, \widetilde{d}, \widetilde{P^{1}}\right), \widetilde{P^{2}} ; \widetilde{d}\right)
$$

which basically means that the discrepancy between actual health care of individual $i$ and the level of health care that is defined as optimal for her depends only on $S E S_{i}$ and not on the $e$ characteristics, i.e., on the demographic and preference variables.

The fairness gap is, in this context, defined as the difference

$$
y_{i}-y\left(\tilde{c}, e_{i}\right)=h_{i}-h\left(h c\left(h n\left(\widetilde{S E S}, d_{i}, P_{i}^{1}\right) \widetilde{S E S}, d_{i}, P_{i}^{2}\right), h n\left(\widetilde{S E S}, d_{i}, P_{i}^{1}\right)\right)
$$

Consider now a situation where the fairness gap equals zero for all individuals. For this situation without illegitimate inequality, (34) implies that

$$
t_{i}^{*}+h c^{*}\left(h n\left(\widetilde{S E S}, d_{i}, P_{i}^{1}\right), P_{i}^{2} ; d_{i}\right)=h c\left(h n\left(\widetilde{S E S}, d_{i}, P_{i}^{1}\right), \widetilde{S E S}, d_{i}, P_{i}^{2}\right)
$$

If we now would like to have that generalized egalitarian-equivalence also evaluates that same situation as one without illegitimate inequality, $t_{i}^{*}$ has to be equal across individuals, i.e.

$$
h c^{*}\left(h n\left(\widetilde{S E S}, d_{i}, P_{i}^{1}\right), P_{i}^{2} ; d_{i}\right)=h c\left(h n\left(\widetilde{S E S}, d_{i}, P_{i}^{1}\right), \widetilde{S E S}, d_{i}, P_{i}^{2}\right)-t^{*}
$$


implying that for all values of the demographic and preference variables in the reference circumstance group, actual health care equals ideal health care up to a constant. This interpretation again may help in choosing the best reference values for an application of the fairness gap approach.

\subsubsection{Comparison with the concentration index and with the measurement of racial dis- parities}

The main difference between the methodology described in the previous subsection and the more traditional approaches such as the concentration index or the measurement of racial disparities is that the latter are interested only in the summary measure of the impact of $c$ on $y$, whereas the former (with the exception of the "average EDE" approach) starts with the computation of individual indexes such that the unfair inequalities are precisely the inequalities in these specific indexes. This makes it possible in particular to analyze the situation for subpopulations rather easily. Indeed, both the concentration index approach and the measurement of racial disparities are special cases of the more general methodology.

Concentration index The concentration index approach has been applied to situations in which $y$ is health as well as to situations in which $y$ is health care. In both cases $c$ is interpreted as socioeconomic status and all the other variables are in $e$. As we have seen in section 3.1, the concentration index of $y$ on $c$ (socioeconomic status) is proportional to the coefficient of the linear regression of $y_{i}$ on $r_{i}^{c}$, the rank of $i$ in the distribution of $c$. Suppose that the following function, similar to (3),

$$
\frac{2 \sigma_{r}^{2}}{\mu(y)} y_{i}=\alpha+\beta r_{i}^{c}+\varepsilon_{i}
$$

does describe how $y_{i} / \mu(y)$ is effectively produced, and that $\varepsilon_{i}$ triggers only legitimate inequalities. Of course, the subtle ethical arguments that have been put forward in the previous subsection cannot be captured by this simple reduced form approach. In addition, note that it is not applicable when $c$ is multidimensional and cannot be unambigously ordered, and that we have to assume that the $y$ function is linear in $r_{i}^{c}$. Both assumptions are not needed in the more general approach of equality of opportunity.

However, if we accept the restrictive assumptions that $c$ indeed can be ordered unambiguously and that the linear approximation is acceptable, the concentration index is closely related to the measures discussed in the previous subsection, as they all focus on $\hat{\beta}$. Indeed, $E\left(y_{i} / \mu(y) \mid c_{i}\right)$ is proportional to $\hat{\alpha}+\hat{\beta} r_{i}^{c}$, and the difference of these terms across individuals $i$ and $j$ is equal to $\hat{\beta}\left(r_{i}^{c}-r_{j}^{c}\right)$. The concentration index then directly reflects the degree of inequalities in $E\left(y_{i} / \mu(y) \mid c_{i}\right)$, implying a perfect 
concordance with the "inequality of means" approach on how to compare distributions. The linearity of the function moreover implies that the other approaches agree with the inequality of means on how to measure inequalities across individuals. The "average EDE" approach will evaluate the differences of expressions $\hat{\alpha}+\hat{\beta} r_{i}^{c}+\varepsilon_{i}$ for individuals with equal $\varepsilon_{i}$, obtaining the same formula $\hat{\beta}\left(r_{i}^{c}-r_{j}^{c}\right)$. Direct unfairness will similarly evaluate inequalities in expressions $\hat{\alpha}+\hat{\beta} r_{i}^{c}+\tilde{\varepsilon}$, and the fairness gap will be proportional to $\hat{\alpha}+\hat{\beta} r_{i}^{c}+\varepsilon_{i}-\left(\hat{\alpha}+\hat{\beta} \tilde{r}^{c}+\varepsilon_{i}\right)=\hat{\beta}\left(r_{i}^{c}-\tilde{r}^{c}\right)$, again yielding the same formula for inequalities across individuals.

Obviously, there may be a problem when some $e$ variables are correlated with $c$ but are nonetheless a source of legitimate inequalities (such as age when $y$ is health, or health needs when $y$ is health care). As explained in section 3, this is typically addressed by standardizing the relevant variables. The analysis in the previous section has shown that such standardization is not ethically innocuous, as it involves compensation and reward issues. Direct standardization substitutes $y\left(c_{i}, \bar{e}\right)$, where $\bar{e}$ is the mean of $e$ over the whole population, to $y_{i}$. If the function $y(c, e)$ is increasing in $c$ (relative to the ordering of $c$ that is used in the concentration index), the concentration index for $y\left(c_{i}, \bar{e}\right)$ is the same as the Gini index. Computing the concentration index for $y\left(c_{i}, \bar{e}\right)$ is then equivalent to computing the Gini index for direct unfairness $y\left(c_{i}, \tilde{e}\right)$ with $\tilde{e}=\bar{e}$. Indirect standardization focuses on $y_{i}-y\left(\bar{c}, e_{i}\right)$, which is again strikingly similar to a concept of the previous subsection, namely, the fairness gap. These similarities suggest two important insights. First, since direct unfairness does not satisfy the compensation principle, the same is true for measures based on direct standardization. This makes direct standardization particularly uninteresting for measuring horizontal inequity in health care delivery. Second, indirect standardization is equivalent to the fairness gap for $\widetilde{c}=\bar{c}$. As described before, this imposes the assumption that "the system gets it right on average". While this assumption is not unreasonable (and is made explicitly in the literature), we have shown in the previous section that it is possible to generalize the approach for other, equally or more attractive, assumptions about the "ideal" health care function (see, e.g., (28)).

When the computation of the concentration index differs from the application of suitable inequality measures to direct unfairness or the fairness gap, for instance because the measure of individual advantage is not comonotonic with $c$, it appears better to rely on an inequality measure. As made clear by Bleichrodt and van Doorslaer (2006), the concentration approach gives absolute priority to individuals with a lower $c$, independently of their personal advantage, which is hard to defend (see section 3). It makes more sense to give priority to individuals who are worse-off according to the relevant measure of advantage, given that this measure is tailored to highlight the benefit due to $c$. A natural solution is then to calculate 
the inequality in the measures of partial advantage as described in the previous subsection. Similar remarks can be made about the choice between the concentration curve applied to $y$ (or its standardized variant) and the Lorenz curve applied to direct unfairness, the fairness gap, or similar measures of partial individual advantage.

Racial disparities Let us now turn to the analysis of racial disparity. Disregarding for simplicity differences in preferences, the approach described in section 3.2 can be translated in our more general setting by interpreting $y_{i}$ as medical consumption and $c_{i}$ as race (B or $\left.\mathrm{W}\right)$. The special structure of the problem (with the IOM definition of racial disparities) requires some additional notation. We will say that the vector $e$ has two components, socioeconomic status $e_{i}^{1}$, for which the correlation with $c_{i}$ generates illegitimate inequalities, and health needs $e_{i}^{2}$ for which the correlation with $c$ does not generate illegitimate inequalities. Note that in this approach correlation between $c_{i}$ and $e_{i}^{1}$ is sufficient and that it is not necessary to impute causality.

Remember that the "inequality of means" approach, as usually applied, would simply compute the difference of average values of $y$ between $B$ and $W$. This is not acceptable because in this setting the correlation between $c$ and $e^{2}$ should be accounted for, i.e., differences in health care needs do not create illegitimate inequalities. It can be shown, however, that there is a close link between racial disparity and the concepts of conditional equality (direct unfairness) and egalitarian-equivalence (fairness gap). Since racial disparity in the first place relates to horizontal inequity (remember the IOM definition), it is natural to focus on measures satisfying the compensation principle in the context of health care. We will therefore restrict ourselves to the latter. We will first consider the additively separable and then the more general case.

\section{Additive separability}

Under the assumption of additive separability, we start from (12), which we can rewrite in the present notation as

$$
y_{i}=\alpha+\beta \varphi\left(c_{i}\right)+\gamma \psi\left(e_{i}^{1}\right)+\delta \chi\left(e_{i}^{2}\right)+\varepsilon_{i}
$$

where we let $\varphi(B)=0, \varphi(W)=1$. The measure of racial disparity then becomes (see (15)):

$$
\text { dis }=\widehat{\beta}+\widehat{\gamma}\left(E\left(\psi\left(e_{i}^{1}\right) \mid W\right)-E\left(\psi\left(e_{i}^{1}\right) \mid B\right)\right)
$$

To implement the IOM concept of racial disparity, in which socioeconomic status as such is a legitimate source of inequality and only its correlation with race is problematic, we use the method that 
was described in the previous subsection. Let us therefore define $e_{i}^{k}, k=1,2$, as a function of $c_{i}$ and the fractional rank (percentile) of $i$ in the distribution of $e^{k}: e_{i}^{k}=\eta^{k}\left(c_{i}, \pi_{i}^{k}\right), k=1,2$. The $y$ function then can be written as

$$
y_{i}=\alpha+\beta \varphi\left(c_{i}\right)+\gamma \psi \circ \eta^{1}\left(c_{i}, \pi_{i}^{1}\right)+\delta \chi\left(e_{i}^{2}\right)+\varepsilon_{i}
$$

and the fairness gap becomes

$$
\hat{\beta}\left(\varphi\left(c_{i}\right)-\varphi(\tilde{c})\right)+\hat{\gamma}\left(\psi\left(e_{i}^{1}\right)-\psi \circ \eta^{1}\left(\tilde{c}, \pi_{i}^{1}\right)\right)
$$

If we pick as the reference value $\tilde{c}=W$, this expression reads

$$
\hat{\beta}\left(\varphi\left(c_{i}\right)-1\right)+\hat{\gamma}\left(\psi\left(e_{i}^{1}\right)-\psi \circ \eta^{1}\left(W, \pi_{i}^{1}\right)\right)
$$

It is then immediately obvious that racial disparity is equal to minus the average fairness gap among the Black population. Note that all individual fairness gaps for White individuals are equal to zero by construction.

It is instructive to compare this result with what would be obtained when we consider both race and socioeconomic status to be illegitimate sources of inequality. This is in line with the approach in the previous subsections but not with the measurement of racial disparity in the IOM definition ${ }^{27}$. The fairness gap is then equal to

$$
\hat{\beta}\left(\varphi\left(c_{i}\right)-\varphi(\tilde{c})\right)+\hat{\gamma}\left(\psi\left(e_{i}^{1}\right)-\psi\left(\tilde{e}^{1}\right)\right) .
$$

If $\tilde{c}=W$ and if the distribution of $e^{1}$ among the White is taken as the reference $\tilde{e}^{1}$, as in the expected form of the fairness gap defined in (30), the fairness gap becomes

$$
\hat{\beta}\left(\varphi\left(c_{i}\right)-1\right)+\hat{\gamma}\left(\psi\left(e_{i}^{1}\right)-E\left(\psi\left(e_{i}^{1}\right) \mid W\right)\right),
$$

so that racial disparity is again equal to minus the average fairness gap among Blacks. Note that the assumptions about the reference values imply that the "ideal" level of health care is defined such that the system gets it right on average for the White. Indeed, the average fairness gap among the White is equal to zero. However, the individual fairness gap for a White individual will only be zero if $\psi\left(e_{i}^{1}\right)=E\left(\psi\left(e_{i}^{1}\right) \mid W\right) .^{28}$

\footnotetext{
${ }^{27}$ It would probably be the favourite approach if one follows the Kawachi et al. (2005) criticism on measuring racial disparity.

${ }^{28}$ Analogous results are obtained if we take $\tilde{c}=B$ and the distribution of $e^{1}$ among the Black as the reference $\tilde{e}^{1}$. Racial disparity then equals the average fairness gap of the White. This close analogy only holds under the assumption of additive separability.
} 
Of course, while disparity is minus the average fairness gap among the Black in both cases, the choices of the reference value and therefore, the assumptions about what is the "ideal" reference level of health care do differ. In the first (IOM) case we assume that the ideal level of health care for any Black is that of a White with the same health care needs and occupying the same fractional rank in the (White) distribution of socioeconomic status as the Black is occupying in the Black distribution. All individual fairness gaps for White individuals are then equal to zero by construction. In the second case we assume that the system gets it right on average for the White. Of course, since SES now is an illegitimate source of inequality, the individual fairness gap may be different from zero for White individuals also. These interpretations stand to reason.

In conclusion, for the additively separable case, racial disparity becomes a special case of the fairness gap and satisfies the compensation condition. Different choices of the reference values will lead to a family of different measures. The interpretations suggested in the previous subsection may be helpful in picking the most adequate measure out of this family. Moreover, with (36) one sees that the fairness gap provides an interesting distribution of individual indexes which makes it possible to analyze in more detail the impact of race on health consumption that is mediated by socioeconomic status. For instance, it may happen that the average fairness gap (and therefore disparity) does not change but that the distribution of the fairness gap improves unambiguously when the impact of race on socioeconomic status becomes more uniform across the distribution. The availability of these individual indexes (and their consistency with the measure of racial disparity) opens a lot of interesting new possibilities.

\section{The non-separable case}

For the non-separable case we focus on the IOM definition of racial disparity and we will compare the different approaches that have been proposed for the non-separable case (see section 3.2) to the fairness gap measured as

$$
y_{i}-y\left(\tilde{c}, \eta^{1}\left(\tilde{c}, \pi_{i}^{1}\right), e_{i}^{2}\right)
$$

The "rank and replace" method (see (18)) gives in the present notation

$$
E\left(y_{i} \mid W\right)-\iint y\left(B, e^{1}, \eta^{2}\left(W, \pi^{2}\right)\right) f\left(e^{1}, \pi^{2} \mid B\right) d e^{1} d \pi^{2}
$$

and

$$
f^{*}(e)=f\left(e^{1}, F\left(e^{2} \mid W\right) \mid B\right) f\left(e^{2} \mid W\right)
$$


Consider the fairness gap for $\widetilde{c}=B$ which gives

$$
y_{i}-y\left(B, \eta^{1}\left(B, \pi_{i}^{1}\right), e_{i}^{2}\right) .
$$

This implies that the fairness gap is zero for every Black. The average fairness gap among the White becomes

$$
E\left(y_{i} \mid W\right)-\iint y\left(B, \eta^{1}\left(B, \pi^{1}\right), e^{2}\right) f\left(\pi^{1}, e^{2} \mid W\right) d \pi^{1} d e^{2} .
$$

Eq. (38) shows immediately that choosing $\widetilde{c}=B$ boils down to transforming the distribution of $e^{1}$ for Whites imagining what would happen to them if they were treated like Blacks. The average fairness gap among the White population coincides with the measure of racial disparity (37). Indeed, the second term in (38) is equivalent to computing $\int y\left(B, e^{1}, e^{2}\right) f^{*}(e) d e$ with

$$
f^{*}(e)=f\left(F\left(e^{1} \mid B\right), e^{2} \mid W\right) f\left(e^{1} \mid B\right)
$$

Moreover,

$$
f\left(e^{1}, F\left(e^{2} \mid W\right) \mid B\right) f\left(e^{2} \mid W\right)=f\left(F\left(e^{1} \mid B\right), e^{2} \mid W\right) f\left(e^{1} \mid B\right)
$$

is equivalent to

$$
f\left(F\left(e^{2} \mid W\right) \mid e^{1}, B\right)=f\left(F\left(e^{1} \mid B\right) \mid e^{2}, W\right),
$$

which holds true because the distribution of percentiles is uniform. Therefore, under the rank and replace method, and under the reference parameters specified above, the measure of disparity coincides with the average difference between Whites and Blacks of the fairness gap. The possibility to take other reference parameters in the fairness gap approach may suggest interesting alternative measures of disparity.

The analogy between the concepts from the equality of opportunity approach and racial disparity is much less clear for the method of propensity scores with rank-and-replace correction.

\subsubsection{Conclusion}

In conclusion, the constructive contributions to the measurement of socioeconomic or demographic inequalities in health and health care that one can make by looking at the formally similar theory of equal opportunities are the following:

- It is possible to construct distributions of individual partial advantage which provide a richer information than average magnitudes or concentration curves; 
- It is easy to accommodate and compare different ethical approaches within a unifying framework; in fact, the most common approaches available in the literature until now (the concentration index and racial disparity) can be interpreted as special cases of this more general approach;

- There is no problem to handle situations with more than one source of illegitimate inequalities, or with a non-linear underlying structure;

- The compensation principle is not satisfied by all approaches and this may be a helpful criterion in the selection - in particular, standardization procedures are not just pure statistics and have ethical implications in this respect;

- The reward principle is also a possible selection criterion, and one can refine the measures in order to introduce ethical preferences about the distribution of health care;

- Inequality measures either coincide with, or are preferable to, concentration measures which require a specific ordering of socioeconomic conditions;

- Numerous variants of measures of inequality and disparity can be constructed from measures inspired by conditional equality and egalitarian-equivalence (such as direct unfairness and the fairness gap). The "average EDE" approach is also another suggestion.

Finally, let us recall an important recommendation regarding methodology. The theory of equal opportunity makes a clear distinction between the function $y$, which is a matter of fact, and the normative evaluation of a particular situation. It is sound to separate the empirical, purely factual, step of estimating the equation $y(c, e)$ from the normative steps having to do with drawing the frontier between $c$ and $e$, and with selecting a particular measure of inequality. Depending on the particular approach that is adopted, more or less information about the function $y$ is needed. For instance, regressing $y$ on $c$, omitting $e$, may be problematic if one needs an unbiased coefficient. Once one draws a clear distinction between the "estimation" and the "evaluation" stage, and one uses inequality measures that are able to accommodate a complicated non-linear structure of $y(c, e)$, it becomes possible to have complete freedom in the estimation stage. Given the essential importance of identifying causal relationships in order to interpret the correlation structure between $c$ and $e$ this seems to be a decisive advantage. 


\subsection{Substantial debate: where to draw the line between "legitimate" and "illegitimate" inequalities?}

The formal apparatus of the theory of equal opportunities can be applied to any partition between $c$ and $e$, and in the previous subsection we have exploited the formal similarity between this theory and issues in socioeconomic health inequalities or racial disparities in health care. But the theory of equal opportunities also suggests that the boundary between $c$ and $e$ should be ethically relevant. For instance, focusing on racial disparities while neglecting the inequalities independently generated by socioeconomic status may give a very partial picture of the prevailing inequities. Focusing on socioeconomic inequalities while neglecting the impact of genetic endowments on inequalities in health may fail to track how unequal the individuals really are in their life prospects. Moreover, the considerations developed about the $c, e$ partition in the normative literature resonate with important debates in health policy about personal responsibility for lifestyle and risk-taking. In this section we discuss some of these general normative issues. In the next section we will show how these philosophical considerations bear on the empirical work.

Our brief review will illustrate some interesting features of the formal framework of the equality of opportunity approach. First, it is sufficiently rich to make a link between different parts of the literature that have remained rather disparate until now. More specifically, it allows for the integration in the formal literature on inequality measurement of some of the philosophical literature on the one hand and of the empirical work on the causes of inequalities in health and health care on the other hand. Second, it is sufficiently flexible to accommodate different ethical and empirical approaches and to compare their implications for the measurement of inequity in health and health care. This is a decisive advantage in a domain where there are many, often conflicting, opinions.

In the first subsection we discuss the two broad approaches in the literature: one holding individuals responsible for their preferences, the other holding them responsible for their choices. We will illustrate the abstract arguments with specific examples taken from the literature on lifestyles and health. ${ }^{29}$ In the second subsection we go more deeply into the treatment of risk and uncertainty. To avoid confusion, it is useful here to add a point of terminology. Mainly in the philosophical literature, what we have called responsibility-sensitive egalitarianism is often denoted as "luck egalitarianism", with "luck" referring to all circumstances that are beyond the control of the individual. This luck egalitarianism is therefore

\footnotetext{
${ }^{29}$ An overview of the economic literature on health behaviour is given by Ruhm and Cawley in chapter 3 of this Handbook.
} 
closely related to what we will call the "choice" approach. In this interpretation "luck" is broader than what most economists would call risk or uncertainty, although of course all circumstances can be seen as events resulting from a kind of natural or social lottery.

\subsubsection{Preference and choice approaches}

The philosophical debate about the "responsibility cut" has seen various positions being developed. The main distinction is that between what could be called the "preference" and the "choice" approaches. The former is already strongly present in the work of Rawls $(1971,1982)$, who argued that treating persons as autonomous moral agents necessarily implies that they should assume responsibility for their goals and their conception of the good life. His difference (or maximin) principle then states that the primary goods (such as income and wealth, and the social bases of self-respect) should be allocated so as to maximize the opportunities of the worst-off in society. Dworkin (1981a,b, 2000) further elaborates on the idea that individuals should be held responsible for their preferences: in his view, an individual cannot sensibly endorse his own preferences about how to conduct his life and at the same time request special help from public institutions on the ground that his preferences are a sort of handicap. Only those who consider their preferences as burdens or cravings, with which they do not identify, can ask for additional resources.

The "preference approach" was attacked by authors such as Arneson (1989), Cohen (1989), and Roemer (1998). They claim that preferences are often the product of upbringing and social influences, for which individuals cannot be held responsible, and they instead advocate the "common-sense" view that individuals should be held responsible only for what they have genuinely chosen, as opposed to what they have inherited from circumstances. In their view one can only be held responsible if one has control.

A second distinction in the literature is somewhat orthogonal to that between the preference and choice approaches. It relates to the justification for introducing responsibility considerations into the evaluation of justice. One approach (implicitly followed by most of the authors mentioned before) accepts that the responsibility cut is a primary data from which one derives the adequate division of labor between social agencies and individuals. In the alternative approach the responsibility cut is derived from other principles having to do with the value of freedom, the importance of political participation, and the like. In this line, Anderson (1999), Sen (1999) and Fleurbaey (2008) defend the view that individuals should be put in good conditions of autonomy and freedom so that they can be the master of their lives and participate fully in social interactions. Yet, when going beyond this general principle, the distinction between the preference and the choice approaches crops up also in this "freedom" approach. Overly 
simplifying, Sen (1999) interprets freedom as the ability to choose from capability sets (and is therefore to be situated rather in the choice-control approach), whereas Fleurbaey (2008) argues that respect for freedom implies respect for personal preferences.

Applied to health, the different approaches would suggest different partitions between factors of inequalities. We will focus on the distinction between the preference and the choice approach. However, the second distinction is also important. While the "freedom" approach is harder to link to a specific proposal in the context of health, one can imagine that it would consider undesirable all inequalities which cannot be traced to the exercise of certain valuable forms of autonomy and freedom. The difficulty would then be to identify what are valuable forms of autonomy, in connection with the population's preferences or with individual choices.

The choice approach In the first approach individuals are held responsible for those characteristics which are under their own control. One can interpret this in a narrow sense and hold individuals simply responsible for all their choices. Yet, this is arguably not in line with the basic intuition of the approach, as choices are also determined by factors that are not under the control of the individuals. Let us illustrate. At first sight, one could say that individuals are to a large extent responsible for their BMI, as this is related to their eating behavior and their level of physical activity. Careful empirical research has shown, however, that cues in the environment may have a great impact on these decisions. The construction of recreational amenities (fitness areas) in Indianapolis led to a significant decrease in the BMI of children visiting pediatric clinics (Sandy et al. 2009). Posting the calorie content of foods and beverages in New York City Starbucks led to a lower consumption of food calories - even extending to the behavior of commuters who later visited other Starbucks establishments in cities where there was no calorie posting (Bollinger et al. 2011). Surely, individual children (or their parents) are not responsible for the availability of fitness areas, nor can individual consumers be held responsible for the information on calorie content that is given in Starbucks. We also know that there is an education gradient in health behaviors, and that this gradient is explained to some extent by differences in cognitive abilities (Cutler and Lleras-Muney 2010). Can it reasonably be stated that individuals control (or choose) their cognitive abilities?

It seems that "genuine control" requires that one corrects for interindividual differences in the environment and also for differences in the choice-making abilities of the individuals. This will be even more relevant if one reasons within the freedom approach to responsibility-sensitive egalitarianism. Yet, this 
brings us on a slippery slope. Is there any room left for genuine control in a deterministic world, if we better and better understand and explain behavior? In a world where the belief in determinism seems great, "it is difficult to expand equality of opportunity in ways that satisfactorily adress the constraining effects of social circumstances, gender socialization, cultural convictions and so on, without undermining the idea of people as responsible agents" (Phillips 2006, p. 19). The choice approach makes the responsibility cut depend on the theory of free will and therefore raises all sorts of thorny metaphysical issues that Rawls and Dworkin deliberately wanted to avoid.

The problem gets a special twist for economists working within the paradigm of rational choice. In this model, genuine choice is an elusive notion, as individual decisions result from a mechanical optimization exercise with a given objective (preferences) and a given set of options (determined by the budget set and possibly additional constraints). All endogenous variables are causally influenced by other variables of the model and therefore cannot embody free will (Fleurbaey 2008, p. 87).

Authors within the choice approach have opted for a pragmatic way out of this deadlock. As we have seen, the most popular solution is that of Roemer (1998). He suggests that every society should be left to resolve the metaphysical debate for itself. "Types" (circumstance classes) are defined a priori, and, since effort is usually unobservable in practice, it is treated as a residual capturing all the differences that are not due to type. The proposed statistical device, which consists in defining that individuals exert the same effort when they lie at the same rank in the distribution of health for their type, makes it possible to take into account the effects of additional conditioning factors in an indirect way. Suppose that we define types on the basis of SES and education. If SES and education are correlated with features of the external environment or with cognitive abilities, the Roemer measure of effort will take into account these correlations. However, this approach is to some extent a black box and does not offer a really convincing answer to the basic philosophical criticism on the difficult delineation of what is control in a deterministic world. Moreover, even from a more mundane perspective, it will at best yield a lower bound of the true inequality of opportunity. Indeed, there will always be unobservable circumstances, such as genetic endowments, so that the a priori partition of the population into classes of circumstances is always coarser than one would like. Taking into account the correlation between types and these unobservable factors is not a satisfactory solution to this problem.

The preference approach The "preference approach" holds individuals responsible for their preferences, i.e., their conceptions of a good life, even if these preferences are not chosen and are not under their 
control. This may lead to a very different evaluation of inequality of opportunity in actual applications. Take the case of two groups in the population with a different cultural background such that individuals belonging to the first group are working (too) hard without taking sufficient time to relax, while individuals from the second group opt for a more healthy lifestyle. In the choice approach one might consider the resulting health inequalities as inequitable, because they simply reflect parental influences during childhood for which individuals cannot be held responsible. In fact, in the Roemer approach differences in preferences that are linked to the type of the individuals will be corrected for when defining the level of "effort". In the preference approach, however, the differences in health and wealth between individuals from these two groups will not be seen as an indication of inequity, if the different lifestyles reflect genuinely different preferences concerning food consumption and physical activity or, more generally, concerning the trade-off between health and wealth.

Since preferences are one of the building blocks of all economic models, the preference approach may seem to fit the economic approach perfectly. Yet, one should be careful about equating immediately "revealed" preferences with the authentic views of the good life that have to be respected. Even more care is needed if one relies on the freedom justification for responsibility-sensitive egalitarianism. The difficulty is then to separate cravings and involuntary addictions (which may be suspected in many unhealthy lifestyles) from genuine preferences. One could even claim that advertising and social pressure may lead to differences in preferences which cannot be seen as authentic.

Let us illustrate again with an example in the domain of health behavior. Chou et al. (2008) have shown that fast food restaurant advertising on television has a significant effect on obesity. According to their estimates, banning this advertising would reduce the number of overweight adolescents in the US by $12 \%$. Our point is not to discuss the reliability of these estimates, nor the desirability of such a ban. What is relevant here is that these results raise the question whether preferences that are induced by television advertising for children (and that may last in their adult life) should be seen as genuine preferences that have to be respected, or rather as cravings caused by the social environment. This is a difficult question indeed, since we run again the risk of landing on a slippery slope. What is the criterion for determining what are authentic preferences? How to avoid ending in a paternalistic approach, in which the analyst ultimately imposes her own views of the good life on the individuals? Surely, such paternalism goes very much against respect for preferences. Empirical work on preference formation may be a valuable input in this kind of debate.

Some philosophers have proposed more general approaches establishing an explicit connection be- 
tween theories of justice and applied issues in health. Segall (2010) focuses on one of the main challenges for luck egalitarianism (in the broader sense of the choice approach), which is the frightening prospect of denying health care to those who can be considered responsible for their health problems. Segall proposes to avoid this prospect by mixing luck egalitarian justice with a sufficientarian component guaranteeing that basic needs should be met for all, irrespectively of their responsibility.

Another proposal for health policy evaluation has been developed by Daniels (1985, 2008). Daniels starts from the observation that Rawls made the simplifying assumption that the members of society are all healthy, and seeks how to extend Rawls' principles of justice in order to make room for the treatment of health inequalities. He rejects the simple solution of extending the list of primary goods governed by the difference principle and proposes to give health a special importance in view of its contribution to people's opportunities. Rawls' principle of "fair equality of opportunities" would, under this extension, require providing health care and managing the other determinants of health in order to guarantee to all, as much as possible, access to normal functioning as a precondition for an autonomous life. Daniels does not make a specific proposal about how to measure health inequalities, but argues that residual health inequalities would be just when all goods governed by the principle of fair equality of opportunities and by the difference principle would be fairly distributed. Obviously, even this does not specify how health priorities should be defined in ordinary health policy decisions. Regarding this issue, Daniels' proposal is procedural, not substantial: decisions should be made through a fair deliberative process. Although this procedural way out is a bit disappointing (after all, it is one of the tasks of philosophers to make a valuable substantial input into this deliberative process), Daniels conveys at least two interesting insights for our purpose. First, the thesis that health inequalities would be fair once all goods have been fairly distributed comes close to the idea that inequalities reflecting different goals in life may be acceptable for a Rawlsian approach. Yet according to Daniels inequity in health cannot be evaluated on its own, but has to be integrated in a broader view on inequity in overall life prospects. This insight will be the leitmotiv of Section 5 of this chapter. Second, this broader view supports some skepticism about the possibility to assign responsibility to individuals for their lifestyle and its health consequences. The priority is to guarantee normal functioning in view of promoting opportunities, and in this perspective the social hijacking made by those who develop risky lifestyles and become a burden for the health care system may be treated by preventive measures of education and incentives rather than by punitive ascriptions of responsibility and a denial of equality in care. 


\subsubsection{Luck and risk}

As we have seen, theories of justice that advocate neutralizing the differential impact over individual success of circumstances that can be considered a matter of sheer luck are branded as "luck egalitarian". However, most authors follow Dworkin (2000) in considering that not all kinds of luck should be submitted to the elimination of differential effects. Thinking of gamblers, Dworkin indeed drew the distinction between "option luck", the kind of luck gamblers deliberately submit themselves to, and "brute luck", the uninsurable luck that even reasonable and prudent people remain vulnerable to. This distinction obviously resonates with many issues in health, as one may think of making a distinction between those who deliberately take a risk by their unhealthy lifestyle and those who are simply the victims of unfortunate unforeseeable events.

However this distinction has proved hard to specify more precisely. Dworkin himself acknowledged that the boundary between cases of option luck and cases of brute luck is fuzzy and a matter of proportion. In particular, when the risky option stochastically dominates the safe options, it is hard to hold individuals responsible for the unequal outcomes they obtain, as even the unlucky made the most prudent choice. Conversely, while it makes more sense to hold the unlucky responsible for submitting themselves to an unfavorable gamble when the risky option is dominated by the safe options, it is no longer clear that a good society should make such unfavorable gambles available when dominating safe options exist. However, even if usual casino gambles are indeed unfavorable (at least in terms of second-order stochastic dominance), the intuition that unlucky gamblers should be left to their fate appears to loom large in the literature on this topic and is motivated, as argued by Dworkin, by a certain respect for risky lifestyles.

More principled and radical attitudes have been defended. At one extreme, Vallentyne (2002) proposes to equalize initial prospects at the beginning of individuals' lives, and to compensate neither option nor brute luck effects, which eliminates the problem of specifying a distinction. One difficulty with this approach is that it is compatible with very large inequalities due to differential luck through life. Moreover, equality of prospects can be artificially organized by lotteries at the beginning of life (as imagined by Harsanyi 1977, babies could be reallocated to families by lot), without apparently making the distribution of final situations more just. At the other extreme, one may argue that all kinds of luck are beyond individual responsibility (Le Grand 1991) and that everyone deserves the expected outcome of his actions but not the differential gain or loss due to the random events that follow. Even if one accepts this view, one can still admit the existence of gambles in cases in which even the unlucky feel better 
off ex post than with the safer options, for instance because the thrill of risk is really what they enjoy (Lippert-Rasmussen 2001).

As a follow up to his distinction between option luck and brute luck, Dworkin proposes a particular way to evaluate health policies and to organize the health care system. The idea is simple. He considers that the ideal health care system would be one in which everyone would be perfectly informed about health risks and about the medical technology, and could take an insurance against adverse health events (including an unfavorable genetic endowment) on the basis of equal budget possibilities and in a perfect insurance market. Such an insurance system would transform all brute luck into option luck. This is obviously not possible but his proposal is to make the real health system mimic this ideal as much as possible, with taxes, public transfers and subsidized provision of care replacing the movements of resources that would be implemented under the ideal (hypothetical) system.

This proposal has been criticized by Roemer (1985) who observed that when adverse health events decrease the marginal utility of income, individuals would ex ante refuse to take an insurance (or would even want to counter-insure themselves and consume more when they are lucky and healthy). This is problematic even when individuals do have the opportunity to insure, because ex post the unlucky are in a bad situation. Their refusal to insure is based on their ex ante ignorance of their final situation. Had they known that they would be unlucky they would have opted for the insurance. Ex ante preferences are based on less information about the state of the world than ex post preferences and the latter should have precedence in social evaluation, as argued by Fleurbaey (2010). This reflects the classical divide in social choice between the ex ante approach that applies a standard social welfare criterion to individual expected utilities and the ex post approach that looks at the possible final distributions of welfare.

Dworkin's insurance device is even more questionable when applied to transfers across different individuals with unequal congenital endowments. It appears grossly unfair to assign lower resources to badly endowed individuals on the ground that if they had had a chance to be better endowed they would have preferred to concentrate their resources on the lucky state. In conclusion, Dworkin's hypothetical insurance proposal, which seems prima facie attractive if one thinks of ordinary insurance against losses which do not affect the utility function, may be unsuitable for an application to health care if adverse health events decrease the marginal utility of income.

What lessons can be drawn from these debates for the measurement of inequity in health and health care? We will discuss some specific methodological points in our overview of empirical applications in the next subsection. From a more general perspective, the most important insight is that measures focusing 
on racial or gender disparities, or on socioeconomic factors of inequality, should not be interpreted as measuring a comprehensive notion of unfairness. They only capture a part of what makes individuals unequally endowed in front of life. Insofar as public policies should be meant to improve the life chances of the worst-off and not just to remove certain specific factors of inequality, it might be a significant improvement for the literature on health inequalities to move the cut between relevant and irrelevant factors of inequality closer to the cut that theories of justice would suggest. More specifically, the impression that socioeconomic inequalities appear especially unjust because they are produced by human institutions should be resisted. The most problematic inequalities are those which are unfair and can most easily be eliminated. It is not necessarily the case that social institutions are more malleable than natural factors, or that their effects are more remediable than the effects of natural causes. If certain congenital handicaps can be easily cured this is a more urgent matter than hardwired inequalities due to early education.

\subsection{Empirical applications}

Empirical applications of the idea of inequality of opportunity in health start from the estimation of some reduced form outcome function $y=f(z)$, where $y$ can refer to health or to health care, depending on the application. As emphasized before, it is important to distinguish clearly this estimation stage from the normative evaluation stage. The empirical analyst should be as free as possible to estimate the best possible explanatory model. The previous sections have suggested at least four important methodological issues that should be considered in going from the estimation stage to the evaluation stage.

The first essential step is the partitioning of the vector $z$ in $(c, e)$. This partitioning will be different depending on the ethical position that is taken. From an applied perspective, the distinction between the control and preference approaches seems the most relevant. While this distinction is clear in principle, there remain some hard nuts to crack for applied analysts. We have already given some examples in previous sections. Suppose one finds a significant effect of religion on the use of health care: does this reflect differences in preferences (e.g., a genuinely different attitude towards physical suffering and death) or a bias due to indoctrination or a lack of correct information? If education has an effect on lifestyle and therefore on health, does this result from different cognitive capacities, from differences in the socioeconomic environment, or from deliberate choices? If there is a significant effect of region, does this capture differences in supply, in needs or in preferences? In fact, many observable variables will 
reflect a mixture of $c$ and $e$ considerations.

This brings us immediately to the second point. In most cases it is extremely useful to estimate the structural model underlying the reduced form $y=f(z)$. Many of the questions concerning the classification of the variables in $c$ or $e$ are ultimately identification questions, that could in principle be answered if a fully specified structural model could be estimated with a complete set of data. Statistical devices like the one proposed by Roemer would then no longer be needed - unless one takes the theoretical position that these statistical devices are not just approximations, but reflect a substantial and ethically attractive position.

Third, at the end of the estimation exercise there will remain some residual unexplained variation. In principle the vector $z$ includes all variables including the error term, and the latter too, therefore, has to be allocated to either $c$ or $e$. It is common practice to simply neglect the residuals and to focus on the deterministic part of the model. Yet this may be highly misleading in this setting — or, formulated more correctly, it implies a specific ethical position. The error term will to some extent capture purely random effects. Including it in $c$ or not then relates to the philosophical discussion on brute versus option luck, or on ex ante versus ex post. However, in applied work the residual will also capture the effect of omitted variables and, in general, unobserved individual heterogeneity - which, on its turn, may be seen as $c$ or $e$ variables. There are no easy solutions, but careful and explicit consideration of the issue is definitely needed.

Finally, we have argued that measuring horizontal equity requires taking an explicit stance on vertical equity. The specification of the "ideal health care" function $h c^{*}\left(h n_{i}, P_{i}^{2} ; S E S_{i}, d_{i}\right)$ is crucial to understand the reward principles underlying the different measures of inequality of opportunity. If one is not happy with the pragmatic assumption that "on average, the system gets it right", one has to think about the additional question of how to specify $h c^{*}($.$) .$

Before turning to applications of the equality of opportunity approach proper, it should be mentioned that recent work within the traditional approaches (concentration index or racial disparities) has explored some interesting possibilities that are highly relevant for measuring inequality of opportunity. One example, that has been mentioned already before, is the measurement of vertical equity by Sutton (2002). He starts from the following specification of the health care equation

$$
h c_{i}=f^{0}\left(h_{i}\right)+\gamma d_{i}+\delta S E S_{i}+\varepsilon_{i}
$$

where $f^{0}\left(h_{i}\right)$ is a non-linear function, approximated in his empirical work by a polynomial. His empirical 


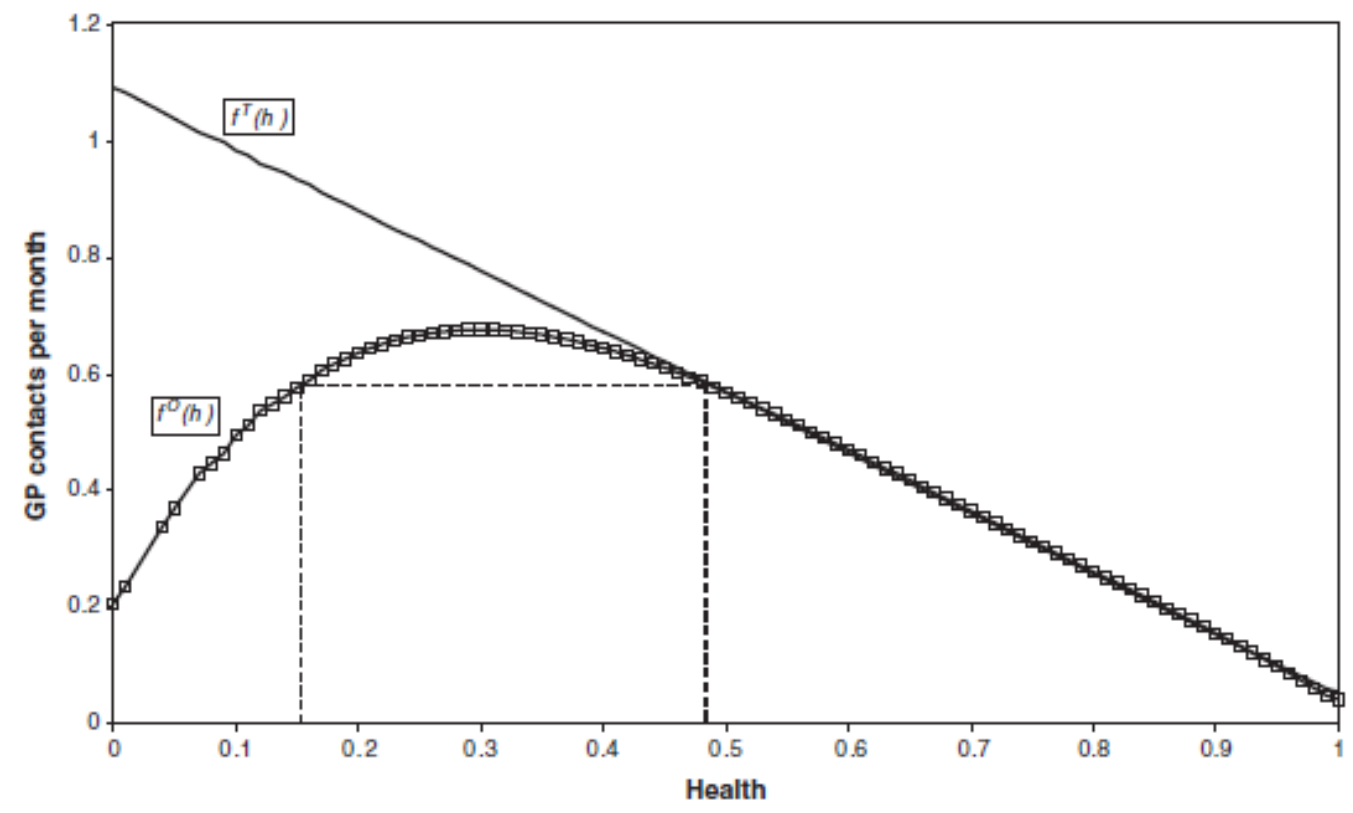

Figure 3: Actual and ideal health care function in Sutton (2002)

estimate of the relationship between health and general practitioner contacts in Scotland is shown in Figure 3. Sutton then claims that this empirical function does not necessarily capture the ideal "target" function $f^{T}\left(h_{i}\right)$ and interprets the difference as capturing "vertical inequity":

$$
h c_{i}=\left[f^{T}\left(h_{i}\right)+f^{V I}\left(h_{i}\right)\right]+\gamma d_{i}+\delta S E S_{i}+\varepsilon_{i}
$$

To identify $f^{T}\left(h_{i}\right)$, he uses Arrow's (1971) insight that an optimal policy should be input-progressive, i.e., that $d f^{T} / d h$ should be negative if worse health is associated with a greater capacity to benefit from health care, which seems a natural assumption. He therefore draws the conclusion that the positively sloped segment in the estimated function $f^{0}(h)$ (for $h<0.31$ ) cannot reflect "ideal" health care. He then reestimates (3) for the range of health values for which $d f^{0} / d h<0-$ and interprets this estimated function as an approximation of $f^{T}\left(h_{i}\right)$. This function turns out to be nearly linear and is shown as the target function $f^{T}(h)$ in Figure 3. It is clear that Sutton needs strong and debatable assumptions to arrive at his results. Yet, further work in this direction could help in getting a better insight in how to specify the (richer) function $h c^{*}\left(h n_{i}, P_{i}^{2} ; S E S_{i}, d_{i}\right)$, that plays an essential role in the measurement of vertical and horizontal equity.

A second interesting contribution is made by Bago d'Uva et al. (2009) in the context of measuring horizontal inequity with the concentration index (see also Van Ourti 2004, and van Doorslaer et al. 
2004). As we noted already, in recent years the awareness has grown that the correction for needs that is necessary for the measurement of horizontal inequity requires an explicit decision about what are the needs and non-needs variables. Recent applied work has implemented sophisticated nonlinear models, for which the linear decomposition method does not work properly. Bago d'Uva et al. (2009) specify the unobserved individual heterogeneity with a latent class model and explain the probabilities of belonging to a given class with individual time-invariant characteristics. The latter can then be partitioned in needs and non-needs variables in the usual way. This allows for a richer interpretation of this individual heterogeneity in terms of the $(c, e)$ partition. As for the remaining unexplained residual, Bago d'Uva et al. (2009) distinguish between what they call the "conventional" and the "conservative" approach. In the former all residual variation is assumed to be determined by non-needs factors, in the latter only the inequality that results from the observed systematic association between income and non-need factors is considered inequitable, i.e., the residual variation is seen as capturing needs. These two approaches can therefore be seen as the two extreme cases in which one interprets the residual in the health care equation as fully $c$ or fully $e$ respectively. The distinction turns out to be empirically relevant, with the conservative approach giving higher estimates of socioeconomic inequity. The treatment of the residuals does indeed matter.

Let us now turn to some explicit applications of the equality of opportunity approach in the health context. In these applications "circumstances" are usually defined to include the socioeconomic background and, more specifically, the childhood circumstances of the individuals. Lifestyle variables (such as smoking, eating behavior, physical activity) are seen as effort variables. The classification of other variables (e.g., educational level or region) is less clear. The literature does not relate explicitly to the philosophical approaches that we described in the previous section. It often implicitly accepts the choice approach and makes use of the Roemer statistical approximation.

A first set of empirical results gets its theoretical underpinning in the treatment of luck by Lefranc et al. (2009). These authors introduce luck explicitly as a third factor, in addition to effort and circumstances, claiming that "luck may appear as a fair source of inequality provided that it is decorrelated from circumstances, in short, even-handed" (p. 1190). Defining the distribution of outcome conditional on circumstances and effort as $F(y \mid c, e)$, they therefore claim that equality of opportunity is satisfied iff: $\forall\left(c, c^{\prime}\right) \forall e, F(y \mid c, e)=F\left(y \mid c^{\prime}, e\right)$. In the terminology introduced before, this implies an ex ante approach in which individuals are not compensated for being unlucky. The condition can be simplified if one accepts the Roemer approach with effort defined as the rank of the individual in the outcome distribution of her 
own type, i.e., if effort is the residual after having taken circumstances in account. Under these conditions equality of opportunity is satisfied if $F(y \mid c)=F\left(y \mid c^{\prime}\right) \cdot{ }^{30}$ As we have emphasized before, in most empirical applications it is not possible to observe all relevant $c$ variables and the definition of types will necessarily be coarse. However, it is easy to see that $F\left(y \mid c_{1}\right)=F\left(y \mid c_{1}^{\prime}\right)$ with $c_{1}$ a subvector of $c$ is a necessary (but not sufficient) condition for equality of opportunity. This is still a very stringent condition. Therefore, the authors propose to work with stochastic dominance conditions. They claim that there necessarily is inequality of opportunity if $F\left(y \mid c_{1}\right)$ first-order dominates $F\left(y \mid c_{1}^{\prime}\right)$, i.e., if $F\left(y \mid c_{1}\right) \leq F\left(y \mid c_{1}^{\prime}\right)$ for all values of $y$, with the inequality being strict for some values of $y .^{31}$

This approach is implemented by Trannoy et al. (2010) and by Rosa Dias (2010) with data for France and Great Britain respectively. In both cases circumstances are defined in terms of the characteristics of the parents. Figure 4 illustrates the French results with circumstances defined as the socioeconomic status of the mother. After formal testing, Trannoy et al. and Rosa Dias conclude that the health distributions for adults that had parents with a higher socioeconomic status dominate the health distributions for adults with parents of lower socioeconomic status. Trannoy et al. (2010) find similar results if circumstances are defined in terms of the health situation of the parents. The facts that types are defined here in a coarse way ánd the application of the extreme criterion of first-order stochastic dominance both strengthen the conclusion that there is inequality of opportunity with respect to health in France and in Great Britain.

Trannoy et al. (2010) and Rosa Dias (2010) realize that this approach in terms of stochastic dominance does not give a specific measure of the degree of inequality of opportunity. They therefore also show a second set of results in which they start from a parametric form of the outcome function $f(z)$. Following Roemer, Rosa Dias accepts that all correlation between effort and circumstance variables should ultimately be interpreted as part of the circumstances. He therefore (deliberately) omits all the $e$ variables from $f(z)$ and estimates the equation

$$
h_{i}=\alpha+\beta c_{i}+\epsilon_{i}
$$

where $\epsilon_{i}$ is a residual and $c_{i}$ is a vector of variables related to the childhood circumstances (such as

\footnotetext{
${ }^{30}$ Lefranc et al. (2009) emphasize that in this approach all random factors should be ascribed to effort. If not, the rank in the outcome distribution for a given type can no longer serve to retrieve effort. One may wonder why these authors introduce luck as a "third" factor, given that circumstances cover all variables for which individuals are not held responsible, and effort covers the rest. It is simpler to understand that they put all the variables that appear as random in the data in the effort category.

${ }^{31}$ Lefranc et al. (2009) also consider the case of second-order stochastic dominance. Health applications have focused on first-order dominance, however, mainly because of the ordinal nature of the health variables used.
} 


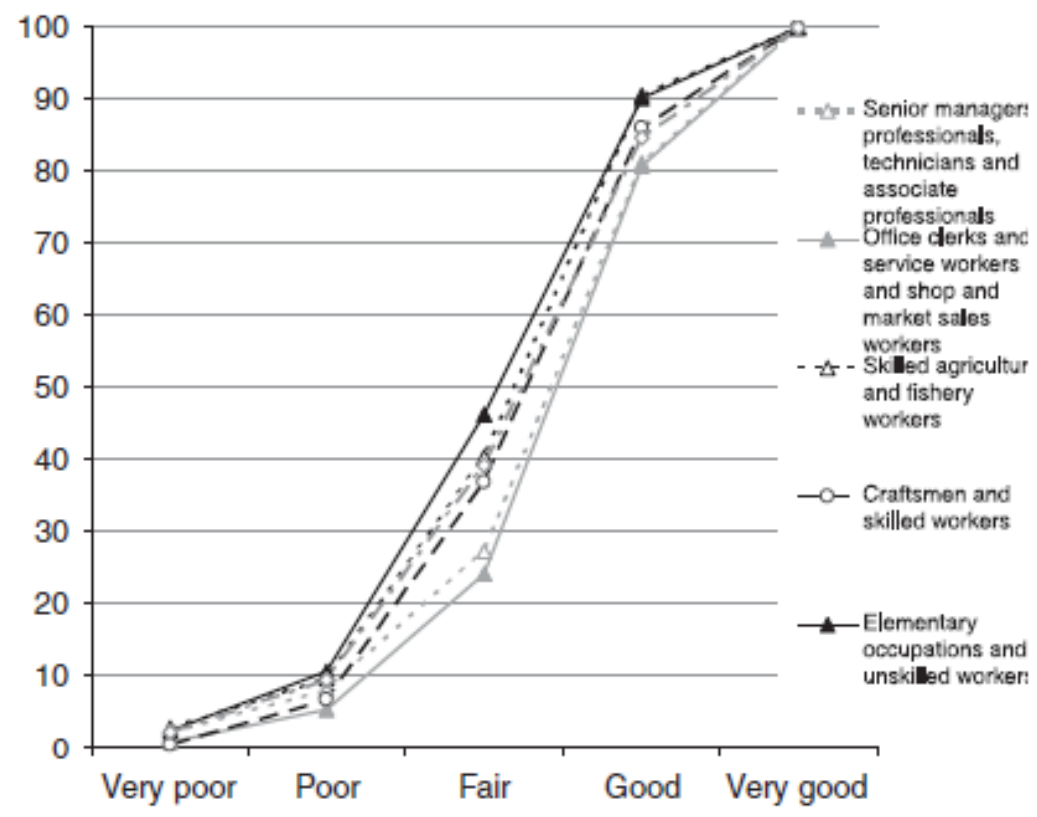

Figure 4: Cumulative distribution functions of self-assessed health according to socio-economic status of mother (Trannoy et al., 2010)

smoking behavior, health and socioeconomic status of the parents). He then computes the pseudo-Gini ${ }^{32}$ coefficient for $\widehat{h}_{i}=\widehat{\beta} c_{i}=h_{i}-\widehat{\epsilon}_{i}$. This is basically "direct unfairness" (conditional equality) as proposed by Fleurbaey and Schokkaert (2009) with the residual $\epsilon_{i}$ implicitly treated as the only effort variable, independent of circumstances $c_{i}$ by construction, and put at its (mean) reference value zero. Rosa Dias (2009) finds that the pseudo-Gini applied to $\widehat{h}_{i}$ is between $21 \%$ and $26 \%$ (varying over the waves) of the pseudo-Gini applied to $h$. Trannoy et al. (2010) interpret education $\left(E D_{i}\right)$ and socioeconomic status as effort variables but clean them for the correlation with circumstances (socioeconomic situation and health of parents) by estimating the following model:

$$
\begin{aligned}
E D_{i} & =\alpha_{0}^{a}+\alpha_{1}^{a} d_{i}+\beta^{a} c_{i}+u_{i}^{a} \\
S E S_{i} & =\alpha_{0}^{b}+\alpha_{1}^{b} d_{i}+\beta^{b} c_{i}+\eta^{b} \widehat{u}_{i}^{a}+u_{i}^{b} \\
h_{i} & =\alpha_{0}^{c}+\alpha_{1}^{c} d_{i}+\beta^{c} c_{i}+\eta^{c} \widehat{u}_{i}^{a}+\theta^{c} \widehat{u}_{i}^{b}+u_{i}^{c}
\end{aligned}
$$

where $\widehat{u}_{i}^{a}$ and $\widehat{u}_{i}^{b}$ are the generalised residuals from the binary probit models (40) and (41). They then compare the Gini and the Erreygers index for $h_{i}$ and for the hypothetical situation where all individuals

\footnotetext{
${ }^{32}$ The use of the pseudo-Gini is necessary because $h$ is measured by self-assessed health, measured on a discrete ordinal scale.
} 
have the health situation $h_{i}^{*}$ corresponding to the best possible circumstances. If all individuals had been fortunate enough to grow up with the best circumstances the Gini coefficient would decrease by $57 \%$ and the Erreygers index by $44 \%{ }^{33}$

These applications are based on Roemer's idea that effort should be purged of any contamination coming from circumstances. Jusot et al. (2010) compare the Roemer approach with what they call the Barry approach, named after the philosopher Brian Barry who claimed (as summarized by Roemer 1998) that "the fact that generally high levels of effort were due to familial pressure does not make their having expended high levels of effort less admirable and less deserving than it would have been absent such pressure". The Roemer and Barry approaches are obviously closely linked to the choice and preference views respectively. Jusot et al. (2010) use French data on subjectively assessed health (a binary variable, taking the value 1 for individuals reporting to be in good or very good health) and include childhood circumstances in $c_{i}$ and lifestyle variables (smoking and eating behavior) in $e_{i}$. They then calculate predicted latent health status using a probit model. In Barry's context, predicted health status is calculated as

$$
\widehat{h}_{i}^{B}=\widehat{\alpha}_{0}^{B}+\widehat{\alpha}_{1}^{B} d_{i}+\widehat{\beta}^{B} c_{i}+\widehat{\eta}^{B} e_{i}
$$

In Roemer's context the approach is similar to that in Trannoy et al. (2010), with

$$
\widehat{h}_{i}^{R}=\widehat{\alpha}_{0}^{R}+\widehat{\alpha}_{1}^{R} d_{i}+\widehat{\beta}^{R} c_{i}+\widehat{\eta}^{R} \widehat{u}_{i}
$$

where $\widehat{u}_{i}$ stands for the generalized residuals from the probit model linking lifestyles to circumstances, i.e.,

$$
e_{i}=\lambda+\delta c_{i}+u_{i}
$$

They then use the variance decomposition (Shorrocks 1982) to split the inequality in $\widehat{h}_{i}^{B}$ and $\widehat{h}_{i}^{R}$ into its different components. Note that their focus on predicted health means that they neglect the contribution of luck to total inequality. It turns out that the differences between the Roemer and Barry approaches are minimal. While the contribution of circumstances to total inequality is $46.4 \%$ in the former, it is still $45.7 \%$ in the latter.

Jusot et al. (2010) remain within a reduced form approach. However, if there is sufficient information about effort and circumstances, it may be worthwhile to work with a fully specified structural model

\footnotetext{
${ }^{33}$ Fixing a reference value for the circumstances is reminiscent of the fairness gap (Fleurbaey and Schokkaert 2009). The latter however would compute the inequality in $\left(h_{i}-h_{i}^{*}\right)$. In general, this does not coincide with the difference between the inequality in $h_{i}$ and that in $h_{i}^{*}$.
} 
$y=f(c, e)$. Estimating such a full structural model makes it possible to interpret carefully the correlation between $c$ and $e$ variables. This may give useful insights into how to classify variables (such as education or region) that obviously reflect a mixture of different underlying mechanisms. Moreover, a structural model is definitely needed if we want to link the measurement of inequity to the deeper philosophical debate on legitimate and illegitimate causes of inequality. For this purpose we need to be able to identify "genuine control" and "authentic preferences". In this regard, the treatment of unobserved heterogeneity is particularly relevant. As Deaton (2011) writes: "Facts and correlations, without an understanding of causation, are neither sufficient to guide policy nor to make ethical judgments. (...) It is possible that an inequality that might seem to be prima facie unjust might actually be the consequence of a deeper mechanism that is in part benevolent, or that is unjust in a different way".

Until now, the introduction of structural models in the literature on inequality of opportunity has been rare. One good example is Balia and Jones (2011). They specify a latent factor model for the initiation of smoking, cessation and mortality and show that parental smoking plays an important role in the dynamics of smoking and indirectly affects mortality. As in Bago d'Uva et al. (2009), analysis of the latent factors makes it possible to get a better insight into the relative importance of unobserved differences in preferences. Balia and Jones (2011) analyse the predictions of two hypothetical scenarios: one in which everyone is given the best circumstances with respect to parental smoking and another one in which everyone is given the highest level of effort, i.e., nobody has started smoking. Average predicted median lifespan is 79.5 in the actual data, and becomes 82.2 and 86.0 in the best circumstances and the best effort scenarios respectively. The inequality in predicted lifespans, as measured by the Gini, is 0.041 in the actual data, 0.039 in the best circumstances scenario and 0.027 in the best efforts scenario. The contribution of circumstances to inequality is therefore relatively small. This is easy to explain: the level of "parental smoking" is only one (perhaps rather minor) aspect of circumstances.

If we want to exploit the full richness of the equality of opportunity framework in empirical work, the analysis of structural models is a promising way to go. This is all the more true as the literature on the causes of smoking and obesity is booming (see the chapter by Ruhm and Cawley in this volume). Moreover, the availability of richer datasets has recently led to a rapid growth in the literature on the effects of childhood circumstances on adult health and wealth (Case et al. 2002, 2005, Currie and Stabile 2003, Currie 2009, Rosa Dias 2010, Van den Berg et al. 2006). This work makes it possible to analyse carefully the relative importance of the different pathways linking childhood circumstances to adult health (ranging from direct fetal influences to the indirect effect throught educational performance and adult 
socioeconomic status). It seems very natural to integrate the empirical insights from this literature into the normative framework sketched in the previous section.

\section{Why care about equity in health? Health and well-being}

In the previous sections we have followed the health economic literature and focused on inequity in health and in health care per se. One might wonder, however, why society should be interested in these partial inequities. Let us first consider inequity in health. If (as seems reasonable) individuals are mainly concerned about overall well-being and if different dimensions of well-being are to some extent substitutable, then "a state of affairs in which those who are otherwise worse off are healthier than those who are otherwise more fortunate is more just rather than less just than a state of affairs which is exactly the same except that health is equally distributed" (Hausman 2007, p. 50). An exclusive focus on health (or, for that matter, any other dimension of well-being) could then lead to misleading conclusions about overall inequity. Rejecting the idea of substitutability leads us into specific egalitarianism (Tobin 1970) with as ultimate requirement that equity should be achieved for all dimensions separately. Yet this requirement is extremely demanding and seems to neglect the fact that "people live differently and encounter different environments... making egalitarianism into a monstrous fantasy of utter uniformity" (Hausman 2007, p. 58). A focus on each dimension separately also goes strongly against the spirit of the theories of equality of opportunity which deliberately leave scope for choice and/or for differences in preferences.

All this does not imply that it is uninteresting to measure inequity in health. First, there is a (perhaps weak) pragmatic argument. The large stream of policy documents on the topic suggests that policy makers are concerned about health inequity, and surveys show that the same is true for the population at large. Second, in line with the ideas of Daniels (2008), one can take the position that health is perhaps not a dimension like the others but has a special importance in view of its contribution to people's overall opportunities - this, however, should be reflected in a suitable measure of overall well-being. Third, collecting information on inequity in health may correct the imbalance in a large part of the economic literature that reduces inequity in overall well-being to inequity in income or material welfare. As an example, adding information on socio-economic inequity in health usually shows that overall inequity in well-being is larger than what one might suspect by looking at income only. It is probably this concern for cumulative deprivation, with the poorest being at the same time less healthy, that motivates the scientific 
literature and the policy interest in socio-economic inequities in health. Of course, the information on inequities in health would be an equally valuable complement to the information on income inequality if the results went in the other direction. Yet in some cases evaluating the distribution of overall well-being (capturing both health and material welfare) is a more natural way of analysing the issue of cumulative deprivation.

A somewhat different story can be told about inequity in health care. Nobody will argue that health care as such is a positive outcome that should appear in the list of desirable dimensions of individual well-being. Equity in health care should be seen in the first place as an instrumental objective — it is important in so far as it contributes to equity in health. One can extend this instrumental reasoning by considering the relevance of equity in health care for other relevant dimensions of individual well-being, such as the feeling of being treated in a fair way by society. Denying health care to people suffering from illness and pain may be seen as a particularly severe infringement on their human dignity, independent of the health effects. Of course, broadening the scope of relevant dimensions of well-being to include respect for human dignity brings us close to the view that society is not only concerned about outcomes, but also about the process through which these outcomes are reached. Equity in health care may obviously be taken up as an important element in such process-sensitive view of justice (Sen 2002).

While inequity in health and in health care remain interesting research topics, a focus on overall wellbeing is necessary to put the partial results on health inequity into a broader perspective. In this section we will therefore consider the relationship between equity in health (care) and equity in well-being. We first look briefly at the literature on equity in finance from this perspective. We then discuss the problem of evaluating the inequality in the bidimensional (income, health) vectors of different individuals.

\subsection{Equity in finance}

In most societies there is a widespread conviction that health care is not a commodity like other commodities, because health care expenditures are largely imposed on individuals, rather than freely chosen. It follows that the financial burden should not disproportionately rest on those who suffer from illness, i.e., that it should be largely independent of the health risks. Many studies on equity in the financing of health care go even further and investigate whether health care financing is linked to ability to pay, partly because there seems to be a widespread commitment to this principle among policy makers (Wagstaff and van Doorslaer 2000). A financing structure is then called progressive if health care expenditures take 
a larger part of income for the rich than for the poor and regressive in the opposite case.

Comparative studies have investigated the degree of progressiveness of overall health care financing in different countries (van Doorslaer et al. 1999, Wagstaff and van Doorslaer 2000, De Graeve and Van Ourti 2003). The ethical relevance of this concept of "progressiveness" is not completely clear, however. Look at the simple example of Table 5. Countries A and B have the same pre-tax income distribution with the poor having an income of 50 and the rich having an income of 150 . Total health care expenditures are 20 , and we assume that health care is free, i.e., that there are no out-of-pocket payments to be paid by the patients. Country A finances its health care expenditures with taxes - i.e., in a progressive way in line with the ability-to-pay principle. Country B has a system of proportional social insurance contributions and its health financing is therefore not progressive. However, in the example country B has a more progressive tax system (used to finance the other government expenditures) than country A. Overall, the total health care expenditures and the net income distribution are identical in countries A and $\mathrm{B}$. In these circumstances it is hard to support the claim that the differences in the financing structure in the two countries have any direct social relevance. What ultimately matters is the overall well-being of the poor and the rich, and these are the same in countries A and B.

Table 5. Health care financing - hypothetical example

\begin{tabular}{|l|c|c|c|c|c|c|}
\hline & \multicolumn{3}{|l|}{ A (tax financed) } & \multicolumn{3}{l|}{ B (social insurance) } \\
\cline { 2 - 8 } & Health contributions & Tax & Total & Health contributions & Tax & Total \\
\hline Poor (50) & 2.5 & 10 & 12.5 & 5 & 7.5 & 12.5 \\
Rich (150) & 17.5 & 70 & 87.5 & 15 & 72.5 & 87.5 \\
\hline & 20 & 80 & 100 & 20 & 80 & 100 \\
\hline
\end{tabular}

The problem with the example in Table 5 is directly linked to the fact that health care expenditures are financed out of the general budget and that the assignment of health care expenditures to different financing sources is in that case largely arbitrary. This issue does not arise when one focuses only on the effects of user charges or out-of-pocket payments of the patients. The empirical work shows that these outof-pocket payments usually are a particularly regressive component of health care financing (Schokkaert and Van de Voorde 2011). This is easy to understand. If the absolute level of health care expenditures were about the same for the poor and the rich (which seems a conservative assumption, given the available evidence on socio-economic inequalities in health), then by construction these expenditures would take up a larger fraction of income for the poorer households. User charges can become "progressive", however, 
if the rich consume disproportionately more health care. The consequences of this are illustrated by the results for 13 Asian territories in O'Donnell et al. (2008). O'Donnell et al. find that out-of-pocket payments for health care are regressive only in Japan and Taiwan, that they are proportional to ability to pay in China, Hong Kong, Korea, Kyrgyz and the Punjab, and that they are even progressive in Bangladesh, Indonesia, Nepal, the Philippines, Sri Lanka and Thailand. However, this mainly reflects that the poor in these so-called "progressive" countries receive less health care since they simply cannot afford to pay and therefore forgo treatment. This suggests that looking at the financing side in isolation may be very misleading in a situation of underconsumption. It is necessary to consider simultaneously (equity in) provision and (equity in) financing.

We therefore agree with the conclusion that "rationalizing the widespread commitment to the abilityto-pay principle in health care financing appears to be harder than might at first be imagined" (Wagstaff and van Doorslaer 2000, p. 1818). From our perspective the findings suggest that one should indeed reason within a broader concept of well-being, including provision (health and health care) and net material consumption as two relevant dimensions. This conclusion remains valid when we drop the ability-to-pay principle and consider the less ambitious objective that individuals with health problems should not have to pay themselves for their treatment, i.e., that they should not be confronted with an income shock on top of the health shock. In applying this principle, the recent literature has mainly focused on the relationship between health care expenditures and poverty in developing countries.

Two approaches have been developed (Wagstaff and van Doorslaer 2003, Wagstaff 2008). A first is to look at so-called catastrophic payments, i.e., to situations in which out-of-pocket payments for health care take a (too) large share in the total household budget. Xu et al. $(2003,2007)$ define health care expenditures as catastrophic when they exceed $40 \%$ of household's nonsubsistence spending, which is total spending minus the food spending of the household having the median food share in total household spending in the country. They estimate (based on surveys in 89 countries covering $89 \%$ of the world population) that 150 million people globally suffer financial catastrophe according to that definition. In their study for Asia, van Doorslaer et al. (2007) define catastrophic payments in terms of the share of outof-pocket payments in total household expenditure and in non-food expenditure - and they show results for a range of "threshold" values. More than $5 \%$ of the households have a share of health care expenses larger than $10 \%$ of total household expenditures in Hong Kong, Kyrgyz Republic, Nepal, Taiwan - and more than $10 \%$ of the households have catastrophic expenditures in Bangladesh, China, India, Korea and Vietnam. A second approach defines households as having catastrophic health expenditures if they 
fall below the poverty line when health care expenditures are taken into account but would not be poor without them - the so-called "medical poverty trap". For 11 Asian countries, van Doorslaer et al. (2006) report that an additional $2.7 \%$ of the population under study (not less than 78 million people) ended up below the $\$ 1$-a-day poverty line due to their health care expenditures.

These studies on the poverty consequences of health expenditures yield useful information on the issue of cumulative deprivation. Yet they suffer from similar limitations as the work on the ability-to-pay principle. First, most studies assume that health care expenditures are paid out of current income or out of current non-medical expenditures. In reality households also resort to other coping strategies such as borrowing or selling a part of their stock of financial and physical assets. Taking these strategies into account changes the poverty picture seriously (Flores et al. 2008, Wagstaff 2008). Therefore a complete evaluation would require the use of a full intertemporal model that takes into account the long-run consequences of the depletion of assets. Second, in this context also a full description of an individual's well-being requires a combination of health and income (poverty) information. Individuals can avoid catastrophic health care payments or escape from the "medical poverty trap" either because they are rich enough or because the health care system delivers health care free of charge or, finally, because they are not consuming the health care they need. From a welfare point of view these three situations are very different. In fact, given that out-of-pocket payments have a negative price effect on the demand for health care, raising them could in principle lead to a decrease in the number of people ending in poverty, simply because the consumption of health care decreases. It would be weird to interpret this as a welfare improvement.

One can try to remedy the partial nature of these measures of "equity in finance" in two ways. The first is to complement them with other measures to yield a complete picture. ${ }^{34}$ A study on the poverty effects of health care expenditures could then be complemented with information on equity in health care delivery and in health outcomes, or with measures of income inequality. Yet, there is a danger that the overall picture remains a bit impressionistic, as it is not obvious how to integrate the different pieces of information in a coherent way. The second approach is more ambitious. It consists in evaluating (the inequality in) overall well-being, taking into account that this requires considering different dimensions, including health and income, in an integrated way. Let us now turn to this issue.

\footnotetext{
${ }^{34}$ This position is explicitly taken for example in Wagstaff and van Doorslaer (2003).
} 


\subsection{Health and well-being}

Individuals care about many things - about income or consumption, but also about health, job security, the environment in which they live, the fate of other people, etc. Well-being therefore seems inherently multidimensional. This is for instance explicitly recognized in the work of Sen $(1993,1999)$ on functionings and capabilities, that has already had some influence in health economics (Anand and Dolan 2005). The question then arises whether it is needed and/or possible to aggregate these different dimensions in one index, and if so, how. The economic tradition with its strong utilitarian roots has a tendency to take the position that all these dimensions only matter in so far as they contribute to "utility", but there is a longstanding debate on the measurability of this concept of "utility" and on its interpersonal comparability.

In the first subsection we describe the intuitions behind the construction of so-called multidimensional inequality indices. We then show how the dominance approach relaxes some of the stringent (and arbitrary) assumptions underlying the measurement of multidimensional inequality. Neither the multidimensional inequality measures nor the dominance approach respect the preferences of the individuals concerning the trade-offs between the different dimensions. The recently booming happiness literature claims to do exactly that. However, in the third subsection we argue that, despite its interesting contribution, it suffers from some weaknesses. We finally discuss an alternative concept respecting individual preferences, the so-called equivalent income.

Throughout this section we restrict the analysis for simplicity to two dimensions. We assume that there is a population of $n$ members and we represent the well-being of member $i$ by a two-dimensional vector $\left(y_{i}, h_{i}\right)$, where $y_{i}$ stands for income or for material welfare and $h_{i}$ stands for health. In some subsections it will be more convenient to switch to the notation $\left(x_{i 1}, x_{i 2}\right)$. In that case $x_{i 1}$ stands for income and $x_{i 2}$ for health. The question is then how to evaluate inequality in these vectors $\left(y_{i}, h_{i}\right)$ or $\left(x_{i 1}, x_{i 2}\right)$. The largest part of the literature that is discussed in this section has not been published in traditional health economic journals. However, most authors take health as their prime example of a relevant dimension of well-being in addition to income or consumption.

\subsubsection{Multidimensional inequality indices}

The basic idea of the literature on multidimensional inequality measurement is to keep to the inherently multidimensional nature of well-being, i.e., to refrain from explicitly formulating a scalar index of wellbeing. This implies that the requirements of inequality measurement have to be formulated directly 
for the vectors $\left(x_{i 1}, x_{i 2}\right)_{i=1, \ldots, n}$. In this respect, two important intuitions have been formalized in the literature (for an overview, see Weymark 2006).

The first is an extension of the one-dimensional Pigou-Dalton principle, stating that an income transfer from an individual with a lower income to an individual with a higher income should increase inequality. To translate this idea into a multidimensional context, Kolm (1977) proposed the principle of uniform majorization $(U M)$. Consider the matrix $X$ with the vectors $\left(x_{i 1}, x_{i 2}\right)$ as rows. The condition $U M$ states that there is at least as much inequality in $X$ as in $X^{\prime}=B X$, where $B$ is a bistochastic matrix (i.e., a nonnegative square matrix with all row and column sums equal to 1). The principle of uniform majorization captures the intuitive idea that a mean-preserving reduction in the dispersion yields lower inequality. Imposing $U M$ together with different scaling and separability assumptions leads to natural generalizations of the best known one-dimensional inequality indices (Tsui 1995, for the Atkinson and the Kolm-Pollak measures; Gajdos and Weymark 2005, for the Gini).

A second consideration comes to the fore in the multidimensional context. As suggested already by the quotation from Hausman (2007) in the beginning of this section, we may also be interested in the correlation between the dimensions (Atkinson and Bourguignon 1982). Tsui (1999) has formalized this as the principle of correlation increasing majorization (CIM). Applied to our simplified setting with only two dimensions we say that $X^{\prime}$ is obtained from $X$ by a correlation-increasing transfer if $x_{i 1}^{\prime}=$ $\max \left(x_{i 1}, x_{j 1}\right), x_{i 2}^{\prime}=\max \left(x_{i 2}, x_{j 2}\right), x_{j 1}^{\prime}=\min \left(x_{i 1}, x_{j 1}\right), x_{j 2}^{\prime}=\min \left(x_{i 2}, x_{j 2}\right)$ and $x_{k d}=x_{k d}^{\prime}$ for $d=1,2$ and all $k \neq i, j$. The principle $C I M$ then states that there is at least as much inequality in $X^{\prime}$ as in $X$. Tsui (1999) has shown that $U M$ and $C I M$ are independent principles. He also derived a family of multi-attribute generalized entropy indices that satisfy both.

It is striking that there are hardly any empirical applications of these multidimensional inequality indices in the health economic literature. Still, the work in this domain throws an interesting light on some of the issues that have been raised in the previous sections. Starting from the matrix $X$, one can distinguish two possible aggregation procedures to arrive at an overall judgment. One procedure is to first calculate indices of income inequality $I\left(x_{1}\right)$ and health inequality $I\left(x_{2}\right)$, where $x_{1}$ and $x_{2}$ are the first and the second column of $X$ respectively, and then calculate overall inequality as a function $f\left(I\left(x_{1}\right), I\left(x_{2}\right)\right)$. This reminds us of the idea that different "partial" measures of inequity (e.g., in health and in health financing) have to be brought together to get at an overall picture. The other procedure first defines an index of well-being $v_{i}=v\left(x_{i 1}, x_{i 2}\right)$ for each individual and then calculates overall inequality in these individual indices. It has been shown that these two approaches only yield the same result under 
very restrictive conditions, basically boiling down to the assumption that the correlation between the dimensions does not matter (Dutta et al. 2003). This is easy to understand. A more constructive way of conveying the same message can be derived from the paper by Abul Naga and Geoffard (2003), who show that for the class of relative bidimensional inequality indices, overall inequality can be decomposed into two univariate Atkinson-Kolm-Sen inequality indices (one for income, one for health) and a third statistic which depends on the joint distribution of income and health. This last term drops out if the function $v\left(x_{i 1}, x_{i 2}\right)$ is additively separable, i.e., can be written as $v^{1}\left(x_{i 1}\right)+v^{2}\left(x_{i 2}\right)$. Since this is not a realistic assumption, one has to combine information on income and health at the individual level to obtain a coherent view on social inequalities.

Returning to the basic issue of the relationship between health and individual well-being, it should be emphasized that both principles $U M$ and $C I M$ are formulated on the matrix $X$ without any reference to preferences. They therefore come in conflict with the ethical requirement that individual preferences should be respected (Fleurbaey and Trannoy 2003). Moving from one dimension to more dimensions indeed changes the issue of measuring inequality in an essential way. In a one-dimensional context one aims at measuring inequality in an attribute (income or health) that can be considered to be desirable for everybody: all individuals agree that more income is better than less, and the same is true for health. Ordinal preferences are identical and different inequality measures then implement different ideas about how to measure inequality between individuals, i.e., how to evaluate the distribution of a universally desired attribute. In contrast, in a two-dimensional setting it becomes highly unrealistic to assume that all individuals share the same preferences over income-health bundles. Although it is claimed that multidimensional inequality measures respect the inherently multidimensional nature of well-being, they ultimately impose a specific rule for aggregating the different dimensions. Interpreted in terms of wellbeing, these measures not only assume (implicitly) that everyone has identical preferences, on top of that they pick (again implicitly) one specific formulation of these preferences, without any guarantee that this choice is representative of the population.

The issue of "responsibility" has not been tackled in this literature. In principle it would be possible, however, to substitute for $h_{i}$ the individual measures of advantage that have been introduced in the previous section. 


\subsubsection{Dominance approaches}

The stochastic dominance approach bypasses the problem of arbitrary weighting of the different dimensions by focusing on the cases in which the comparison between two distributions is independent of the particular weighting system chosen. In such cases, the conclusions appear especially robust — the downside is that this can be achieved only for a limited subset of cases. The idea of seeking robust cases is simple, but its implementation is potentially complex, as one may have to make a huge number of calculations, considering many possible vectors of weights with a sufficiently fine grid. Even with computers, this implies a staggering amount of calculus when the applications bear on large samples of populations. The objective of the dominance literature has been to develop simple criteria which can be applied in a reasonable number of steps. The prime example of such a criterion is the Lorenz curve, which is constructed for unidimensional distributions. A remarkable result due to Hardy, Littlewood and Pólya (1952) establishes an equivalence, for the comparisons of two distributions (with the same mean) of a continuous variable in a finite population, between: $(H L P 1)$ a higher Lorenz curve; $(H L P 2)$ a greater social welfare for a wide class of social welfare functions; ( $H L P 3)$ a finite number of permutations and progressive transfers.

The idea of extending this approach to multidimensional distributions has been first explored by Atkinson and Bourguignon $(1982,1987) .{ }^{35}$ The main focus has been on extending the equivalence $(H L P 1) \Leftrightarrow(H L P 2)$ of the Hardy-Littlewood-Pólya theorem, the study of associated transfer principles (i.e., HLP3) appearing much more difficult than in the one-dimensional case (Trannoy 2006, Gravel and Moyes 2011). In line with our assumptions in this section, most of the literature is about the bidimensional case. Two situations can be distinguished: one in which both variables are continuous and a second one in which one variable is continuous and the other is discrete. ${ }^{36}$ The two parts of the literature are relevant to our setting. Income is a continuous variable, while health can sometimes be treated as a continuous variable (QALYs) and sometimes as a discrete variable (e.g., self-assessed health with four or five categories).

\footnotetext{
${ }^{35}$ There is a formally parallel analysis in the theory of risk (e.g., Richard 1975, Levy and Paroush 1974). An overview of the literature on multidimensional stochastic dominance can be found in Trannoy (2006).

${ }^{36}$ The main application motivating the use of a discrete variable is when statistical units are households of different sizes. Household income and household size (or demographic composition, if a distinction is made between adults and children) then form the pair of variables.
} 
Formally, social welfare is assumed to be defined as the sum of utilities:

$$
\sum_{i=1}^{n} U\left(x_{i 1}, x_{i 2}\right)
$$

where $i$ is the individual index running over the population of $n$ members. This apparently utilitarian form involves no more than separability and continuity, because there is no commitment to any particular measure of utility. Indeed, in this literature the function $U($.$) is not necessarily seen as a representation of$ individual preferences, it rather reflects the weighting scheme of the social planner. It can therefore easily be interpreted as a generalization of the specific functional forms that are embodied in the multidimensional inequality measures. While separability and continuity are both questionable assumptions, they are sufficiently popular in welfare economics to make the whole enterprise worthy of interest. Defining a class of social welfare functions under these two assumptions then boils down to defining a class of utility functions $U$.

The idea of stochastic dominance is to derive easily verifiable conditions on the joint distribution $F\left(x_{1}, x_{2}\right)$ (extensions of Lorenz-curve domination) that are equivalent to a higher social welfare for a whole class of utility functions $U$. Here we will not dwell on these particular criteria. They are easy to find in the quoted literature. What is more interesting for our purpose is to examine if the classes of utility functions appear sensible when the first variable is income and the second is health. These classes are characterized by properties of the derivatives of $U$. Let $U_{t}=\partial U / \partial x_{i t}, t=1,2, U_{s t}=\partial U / \partial x_{i s} \partial x_{i t}$, $s, t=1,2$, and so on. When $x_{i 2}$ is a discrete variable taking the values $k=1, \ldots, K$, one can understand the derivatives as $U_{2}\left(x_{i 1}, x_{i 2}\right)=U\left(x_{i 1}, x_{i 2}\right)-U\left(x_{i 1}, x_{i 2}-1\right), U_{12}\left(x_{i 1}, x_{i 2}\right)=U_{1}\left(x_{i 1}, x_{i 2}\right)-U_{1}\left(x_{i 1}, x_{i 2}-1\right)$, $U_{122}\left(x_{i 1}, x_{i 2}\right)=U_{1}\left(x_{i 1}, x_{i 2}\right)-2 U_{1}\left(x_{i 1}, x_{i 2}-1\right)+U_{1}\left(x_{i 1}, x_{i 2}-2\right)$, and so on. Table 6 summarizes the classes that have been studied and for which Lorenz-type criteria have been found. ${ }^{37}$ The superscript $d$ indicates that the second variable is discrete.

\footnotetext{
${ }^{37}$ We ignore the study of first-order stochastic dominance and focus on the extension of Lorenz dominance. Moreover, we do not include the cases in which the marginal distribution of variable 2 is kept fixed (Bourguignon 1989), or in which $U_{2}=0$ for $x_{i 1}$ large (Jenkins and Lambert 1993), because these are less relevant for our problem of evaluating allocations of income and health.
} 
Table 6. Dominance approaches: classes of social welfare functions

\begin{tabular}{lll}
\hline \hline C1: & $U_{1}, U_{2} \geq 0, U_{11}, U_{12} \leq 0, U_{112} \geq 0$ & Moyes (1999) \\
C2: & $U_{1}, U_{2} \geq 0, U_{12}, U_{22} \leq 0, U_{122} \geq 0$ & Moyes (1999) \\
C3: & $U_{1}, U_{2} \geq 0, U_{11}, U_{22}, U_{12} \leq 0, U_{112}, U_{122} \geq 0, U_{1122} \leq 0$ & Atkinson and Bourguignon (1982), Moyes (1999) \\
C4: & $U_{1}, U_{2} \geq 0, U_{11}, U_{22}, U_{12} \leq 0, U_{112}, U_{122} \leq 0, U_{1122} \geq 0$ & Atkinson and Bourguignon (1982) \\
C5: & $U_{1}, U_{2} \geq 0, U_{11}, U_{22}, U_{12} \leq 0, U_{111}, U_{112} \geq 0$ & Muller and Trannoy (2003) \\
C6: & $U_{1}, U_{2} \geq 0, U_{11}, U_{22}, U_{12} \leq 0, U_{222}, U_{122} \geq 0$ & Muller and Trannoy (2003) \\
\hline \hline
\end{tabular}

A class defined with more conditions is smaller and therefore corresponds to a dominance criterion which is more discriminating — but obviously, piling up conditions raises occasions for ethical objections. A simple way to get an intuitive grasp of the conditions on derivatives is to visualize what they imply for the evaluation of a change in the situation of one or more individuals (Moyes 1999). Figure 5 shows changes (the initial position of an individual is represented by a circle, the final position by a star) ${ }^{38}$ which are improvements if the corresponding condition on $U$ is satisfied. Note that a condition on a derivative of order $m$ requires a figure with $2^{m-1}$ individuals.

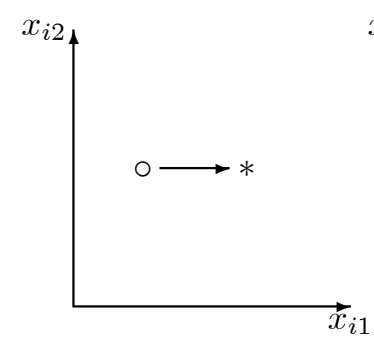

$\left(U_{1} \geq 0\right)$

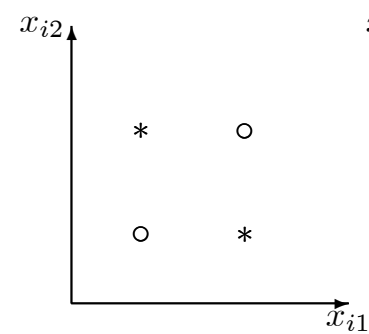

$\left(U_{12} \leq 0\right)$

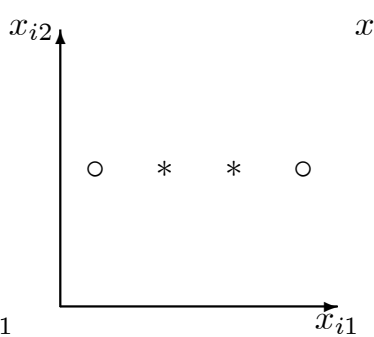

$\left(U_{11} \leq 0\right)$

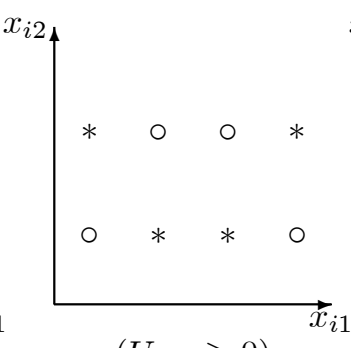

$\left(U_{112} \geq 0\right)$

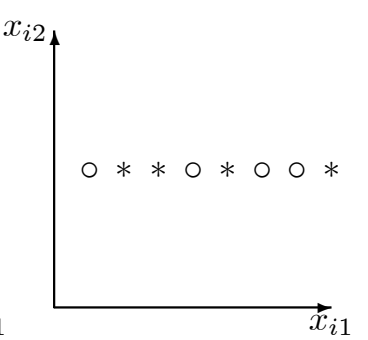

$\left(U_{111} \geq 0\right)$

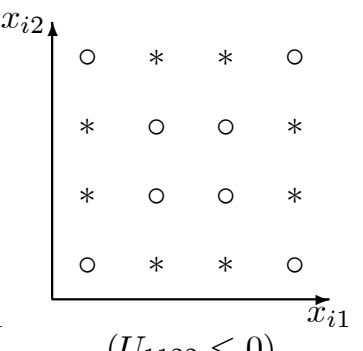

$\left(U_{1122} \leq 0\right)$

Figure 5: Illustration of the conditions on $U$

We are now in a position to examine these conditions and evaluate their relevance in the particular

\footnotetext{
${ }^{38} \mathrm{An}$ arrow illustrates the change for the first graph. Arrows are omitted for the other graphs because, except for the first graph, there are several possible ways to connect initial positions to final positions by arrows.
} 
context in which dimension 1 is income (or consumption) and dimension 2 is health.

- $U_{1}, U_{2} \geq 0$ : it is very plausible to require utility to be increasing in both variables.

- $U_{11}, U_{22} \leq 0$ : it is also plausible to have a decreasing marginal utility in both dimensions. This justifies transferring from the better-off to the worse-off in each dimension. (In the case of health, a "transfer" is just a symbolic expression describing a situation in which one distribution is less unequal than another.)

- $U_{12} \leq 0$ : this crucial condition means that less healthy individuals have greater priority in the distribution of income. As illustrated in the figure, a society in which the rich are sick and the poor are healthy is considered better than a society in which riches and health are concentrated in the same individuals. This reflects the intuition that was also captured by the principle CIM (correlation increasing majorization) in the literature on multidimensional inequality measurement. It goes against a certain utilitarian logic that would consider that healthier individuals are those who can benefit from income.

$-U_{112} \geq 0$ : this condition means that the priority of the sick for the distribution of income decreases as they become richer. As illustrated in the figure, it means that it is more important to reduce the correlation between income and health among the poor than among the rich. It also means that the priority of the poor in the redistribution of income is less strong among healthier people. This condition therefore appears more plausible than the opposite condition $U_{112} \leq 0$.

- $U_{122} \geq 0$ : this condition means that the priority of the sick in the distribution of income decreases among healthier people, and also that the priority of the sick in the distribution of health decreases among richer people. Again this condition does not seem unreasonable in the context of health, and is ethically more plausible than the opposite condition $U_{122} \leq 0$.

- $U_{111}, U_{222} \geq 0$ : this rather plausible condition means that marginal utility decreases less steeply as income (resp., health) increases. Redistribution is less desirable among the well-off than among the badly-off.

- $U_{1122} \leq 0$ : this is the hardest condition to intuitively understand and to assess. It means that the decrease in the priority of the sick as they become richer (healthier) is less steep for healthier (richer, respectively) individuals. As illustrated in the figure, this implies that decreasing the correlation 


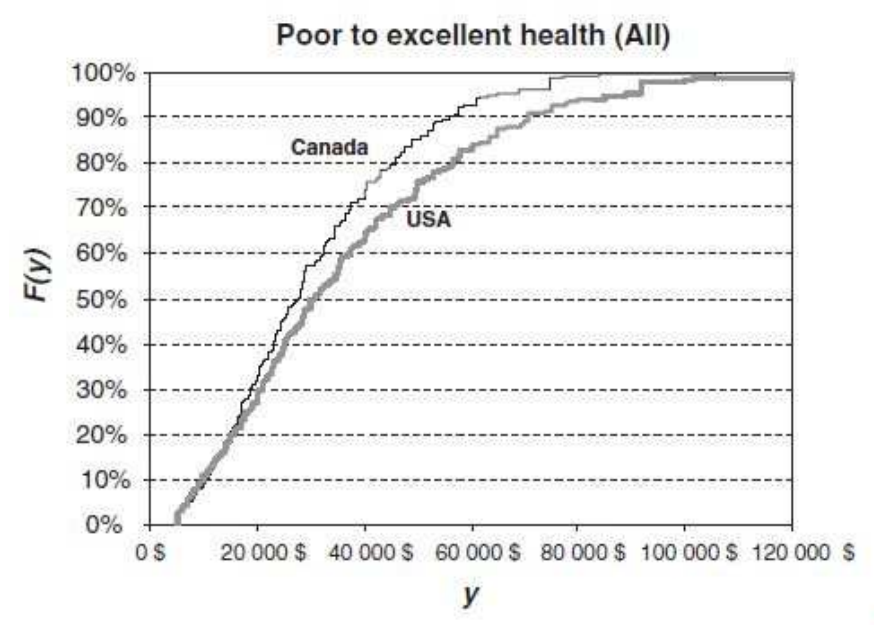

Figure 6: Cumulative income distributions in Canada and in the USA (Duclos and Echevin, 2011)

between income and health is more important among the extremes (sick poor and healthy rich) than among the mixed positions (sick rich and healthy poor). Viewed in this way, it appears clearly more reasonable than the opposite condition $U_{1122} \geq 0$.

In conclusion, these comments suggest that most of the classes are worth considering in the context of income and health. One may, however, have doubts about class C4. Trannoy (2006) argues that C5 corresponds more to the case in which income is used as a tool for the compensation of sickness, while C6 considers using health as a compensation for poverty. Both perspectives seem to make sense, although the former is arguably more natural.

The power of the dominance approaches is illustrated in a recent paper by Duclos and Echevin (2011). They compare the situations of Canada and the USA and also analyse the developments over time in both countries. By way of illustration we show in Figure 6 the cumulative income distributions in Canada and the US, showing that incomes are higher in the US than in Canada - and in Figure 7 the cumulative income distributions when one considers only the individuals in poor or in fair health. Duclos and Echevin (2011) test for the statistical differences between these curves (and similar ones for other health levels) and draw the conclusion that for all utility functions in the class $\mathrm{C} 1$ from Table 6 , the social welfare is higher in Canada than in the US, if one focuses only on those with poor and fair health statuses. This is due to the fact that the worse income distribution in Canada is compensated by a better health distribution, by a lower correlation between health and income, and by lower income inequality in Canada than in the US. Using similar techniques, they also show that social welfare has 


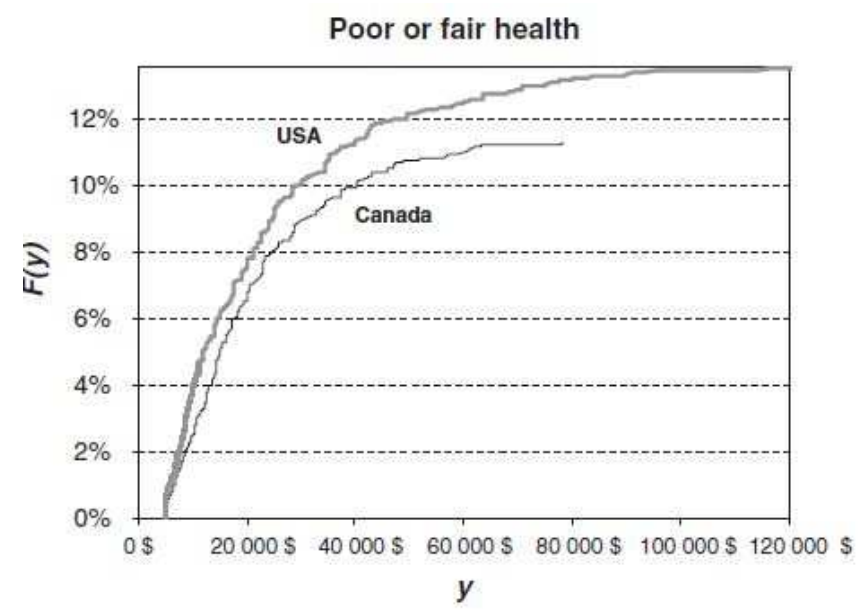

Figure 7: Cumulative income distribution functions in Canada and in the USA (2003) for individuals in poor or fair health (Duclos and Echevin, 2011)

not improved in the US during the decade 1996-2005, in spite of the overall increase in incomes. This is because the US health distribution has deteriorated over time and because this deterioration has not been offset by sufficient increases in incomes for the least healthy. For Canada, the normative evaluation of the change over time depends on which part of the joint health-income distribution is judged to be of greater normative importance.

Finally, let us mention two limitations of the dominance approach. First, dominance is not obtained in all comparisons, so that the ranking of distributions produced by such criteria is always incomplete. More restrictive classes yield less incomplete rankings, but never produce completeness. Second, the underlying social criterion involves a single utility function $U$, thereby ignoring the diversity of individual preferences. Even if a class of utility functions is always referred to in the dominance criteria, the unanimous judgment of a class of social welfare functions $\sum_{i=1}^{n} U\left(x_{i 1}, x_{i 2}\right)$ for a variety of functions $U$ is not the same as a judgment based on a function $\sum_{i=1}^{n} U_{i}\left(x_{i 1}, x_{i 2}\right)$ in which different utility functions for different individuals are used at the same time. The diversity of subjective perspectives across individuals is the focus of the approaches presented in the next subsections. 


\subsubsection{Welfarism: happiness and health}

Social choice theory is famous for Arrow's (1951) impossibility theorem, which has often been interpreted as implying that it is impossible to derive a welfare ordering of social states ${ }^{39}$ on the basis of individual preferences, while at the same time respecting some ethical conditions (such as non-dictatorship). At first sight this impossibility theorem makes the quest for a preference-based evaluation of joint distributions of income and health meaningless. However, possibility results can be obtained by relaxing one or more of Arrow's conditions. In this respect, much research has focused on the axiom of Independence of Irrelevant Alternatives, which requires comparing two alternatives with information about individual preferences which is limited to whether any given individual prefers one alternative or the other. It has been shown that this axiom can be relaxed in two ways, both of which allow to take into account more information about individual well-being. One way, initiated by Sen (1970), consists in introducing interpersonally comparable utility functions and allowing some information about utility levels or differences to play a role in the comparison of two alternatives. The other way, suggested by Hansson (1973) among others, consists in allowing information about indifference curves to be taken into account. In this section we walk the first way.

Within the economic tradition with its strong utilitarian roots, it would seem natural to take utility as an indicator of well being, if the utility function $U_{i}\left(x_{i 1}, x_{i 2}\right)$ were cardinally measurable and interpersonally comparable. For a long time, these assumptions of measurability and interpersonal comparability have been seen as unrealistic. Microeconomists have emphasized that choice behavior can only reveal ordinal preferences and that the utility function representing these individual preferences therefore is only defined up to a monotonic transformation. Recently, however, a growing number of economists have questioned the traditional sceptical attitude regarding stated preferences and accept that individual answers to survey questions are credible as direct measures of happiness or satisfaction with life. The formulations of these questions are different in different surveys, but a typical example would be: "All things considered, how satisfied are you with your life?" There is by now a wealth of evidence for different countries showing that the answers to these questions are reasonably coherent over time and that the measured level of subjective satisfaction is related in a meaningful way to observable characteristics of the situation of the respondents. Some have argued that since we now finally are able to measure utility, we can start testing directly some predictions of economic theory (Frey and Stutzer 2002). Others have

\footnotetext{
${ }^{39} \mathrm{In}$ our simplified context a social state is a collection of income-health bundles for all individuals, i.e. $\left(\left(y_{1}, h_{1}\right), \ldots,\left(y_{n}, h_{n}\right)\right)$.
} 
drawn explicit normative conclusions from these findings in a utilitarian perspective (Layard 2005). ${ }^{40}$

The typical approach in the literature has been the estimation of a so-called "happiness equation" $S W B_{i}=f\left(y_{i}, h_{i}, z_{i}, \varepsilon_{i}\right)$, where $S W B$ stands for subjective well-being, $z_{i}$ is a vector of observable individual characteristics (different from income and health) and $\varepsilon_{i}$ is an error term. A common finding is that of a strong statistical relationship between health and happiness (Graham 2008). Although causality runs in both directions, it is well documented that good health increases the level of happiness and that health shocks - such as serious diseases or permanent disabilities - have negative effects. The effect of health is even more robust than the effect of income. The estimation results make it possible to calculate the marginal rate of substitution between health and income $\left(\partial f / \partial h_{i}\right) /\left(\partial f / \partial y_{i}\right)$, which can also be interpreted as a willingness-to-pay for better health. This willingness-to-pay turns out to be large for chronical diseases. As an example, it is found that for West-German workers hearing impediments are on average equivalent to an income reduction of about $20 \%$, and heart or blood difficulties are equivalent to a $47 \%$ income reduction (Ferrer-i-Carbonell and Van Praag 2002). For a 25 year old Dutch male the monetary valuation of a heart disease ranges from $€ 114000$ to $€ 380000$ depending on the welfare level (Groot et al. 2004, Groot and Maassen van den Brink 2006). Although care is needed because the results depend on the specification of the function $f($.$) and on the precise measure used for S W B$, there is no doubt that the trade-off between income and health shows up clearly in these estimates.

All this suggests that it would be possible to use the individual values of $S W B_{i}$ coming out of the surveys as a direct measure of individual well-being. One could then immediately calculate the inequality in these values and analyse how it relates to (inequity in) health or health care, to socio-economic status and to the income consequences of having to pay for health care. Yet, this would mean that we basically return to utilitarianism. In the social philosophical literature, utilitarianism has come under severe criticism, as exemplified by the extremely influential work of Rawls (1971). This criticism has also been taken up by a large fraction of social choice theorists (inspired among others by Sen 1985). They doubt that subjective utility is really an ethically attractive indicator of well-being.

Sen (1985) raises two sets of arguments. Both are taken up by Loewenstein and Ubel (2008) from a

\footnotetext{
${ }^{40}$ The economic literature on happiness and on how to measure it has become so large that it is impossible to summarize it in a few pages. We therefore can only raise some general points, but we believe that our positive as well as our critical remarks are relevant for all the variants of happiness measurement. Moreover, note that we are only concerned here with measures of overall satisfaction with life, and not with subjective quality of life in the health economic tradition of QALYmeasurement nor with subjective health satisfaction or with self-assessed health. We focus in this section on finding an overall measure of well-being that would make it possible to formalize the trade-off between income and health.
} 
health perspective. First, Sen argues that utilitarianism suffers from "valuation neglect". Valuing a life is a reflective activity in a way that being happy or desiring need not be (Sen 1985, p. 29). People do not only live to feel happy, they care about the meaning that they derive from activities. Non-affective (i.e., cognitive) components of well-being are essential to most individuals. Or, in the words of Loewenstein and Ubel (2008, p. 1801), "experience utility fails to capture a wide range of dimensions of existence that people deeply and legitimately care about". As an example, people care a lot about dying a "good death". Second, serious issues are raised by the phenomenon of adaptation. Sen talks about "physical condition neglect". Loewenstein and Ubel (2008, p. 1799) state that numerous studies have found that people with chronic health conditions as severe as kidney failure or paraplegia report moods that are relatively close to those reported by healthy persons. However, although people may be able to emotionally adapt to a wide range of health conditions, that does not mean that they are indifferent between them. Quite the contrary, despite reporting levels of well-being that are similar to those of healthy persons, people in poor health conditions are willing to make large monetary or non-monetary sacrifices to become healthy again.

The last point brings us back to the distinction between preferences, represented by an ordinal utility function on the one hand, and cardinal happiness measures on the other hand. Take an individual $i$ who prefers $\left(y_{i}, h_{i}\right)$ to $\left(y_{i}^{\prime}, h_{i}^{\prime}\right)$. (We drop the variables $z$ and $\varepsilon$ for convenience). If she answers the happiness question truthfully, then her preference will be reflected in her reported happiness values, i.e., $S W B_{i}=f\left(y_{i}, h_{i}\right)>S W B_{i}^{\prime}=f\left(y_{i}^{\prime}, h_{i}^{\prime}\right)$. This means that the happiness measure corresponds to a cardinalization of the utility function that is representing her preferences. But let us now take two individuals that both prefer $(y, h)$ to $\left(y^{\prime}, h^{\prime}\right)$. It will then still be true that $S W B_{i}>S W B_{i}^{\prime}$ and that $S W B_{j}=f\left(y_{j}, h_{j}\right)>S W B_{j}^{\prime}=f\left(y_{j}^{\prime}, h_{j}^{\prime}\right)$. However, it is very well possible that $S W B_{j}^{\prime} \geq S W B_{i}$, if the aspiration levels of the two individuals differ, e.g., because individual $j$ has adapted to the bad situation $\left(y^{\prime}, h^{\prime}\right)$. In other words, it is possible that individual $j$ does not feel less happy in the incomehealth situation $\left(y_{j}^{\prime}, h_{j}^{\prime}\right)$ than individual $i$ in the situation $\left(y_{i}, h_{i}\right)$, although they both prefer $(y, h)$ to $\left(y^{\prime}, h^{\prime}\right)$. A similar phenomenon can occur if we consider one individual with changing aspirations over time. This reasoning shows a perhaps somewhat surprising result: evaluating individual well-being on the basis of subjective happiness measures does not necessarily respect preferences, because these happiness measures are contaminated by adaptation and differences in aspirations. If we want to respect individual preferences, we have to look for another approach. This brings us to the second escape route from Arrow's impossibility, that consists in using information about individual indifference curves. 


\subsubsection{Respecting preferences: equivalent income}

If using happiness measure does not respect preferences, what does? To get an intuition of what is at stake, consider a simple world in which individuals care only about one magnitude in their life, call it $X$. A life with more $X$ is considered a better life by everyone. Respecting individual judgments about the quality of various lives therefore requires using $X$ as the metric of success in life. Even in this simple unidimensional world, happiness measures do not respect preferences in general because one may have $X_{i}>X_{j}$ but $S W B_{i}\left(X_{i}\right)<S W B_{j}\left(X_{j}\right)$. There is a clear sense in which people care about $X$, not $S W B(X)$, and one should not mistake the evaluation $(S W B(X))$ for what is valued $(X)$. Adopting the $X$ metric, social preferences should then simply be defined as preferences over distributions of $X$.

What happens when life has several dimensions and individuals may have different preferences? Let us return to our setting with two dimensions, income $y$ and health $h$. Intuitively, just as $X$ was a suitable metric in the unidimensional case, something of the same nature as $y$ or $h$ should become the metric in this case. One attractive approach to do exactly this is that of the healthy-equivalent income (Fleurbaey 2005). ${ }^{41}$ It is measured in the same units as $y$, and is defined as the level of income $y_{i}^{*}$ such that the individual is indifferent between his current situation and being healthy with income $y_{i}^{*}$, i.e., between $\left(y_{i}, h_{i}\right)$ and $\left(y_{i}^{*}, h^{*}\right)$, where $h^{*}$ refers to perfect health. There are good reasons to take perfect health as the reference. More specifically, this choice implies that the healthy-equivalent income satisfies the property that individuals with a good health can be compared directly in terms of incomes, independently of their preferences. Indeed, their equivalent incomes are then equal to their ordinary incomes, whatever their preferences may be. This is a rather nice property, as it is intuitive that, when healthy, an individual with more income than another enjoys a better situation, even if their preferences are different.

Figure 8 clarifies the concept. Suppose we want to compare the well-being of individual $i$ in situation

${ }^{41}$ The notion of equivalent income and its interesting history in economics are explained in more detail in Fleurbaey (2009). The concept was first introduced, under the label "money-metric utility", by Samuelson (1974) and Samuelson and Swamy (1974) in the search of an index of quantities that would track individual preferences better than the usual Laspeyres, Paasche and Fischer indexes. It was then used in the analysis of the distribution of household welfare by King (1983) - who coined the expression "equivalent income" - and features honorably in the classical monograph by Deaton and Muellbauer (1980). The fad quickly faded, however, when Blackorby and Donaldson (1988) observed that an additively separable social welfare function using money-metric utilities as the indexes of individual well-being could not be quasi-concave with respect to quantities, therefore violating a basic requirement of inequality aversion. The recent theory of fair allocation provided fuel for a new interest in the notion. As Fleurbaey (2005) shows, it can be seen as a further elaboration of the idea of "egalitarian-equivalence" introduced by Pazner and Schmeidler (1978) and already mentioned in this chapter. 
A with that of individual $j$ in situation B. If we want to respect the preferences of individual $i$, we have to take situations A and A' as equivalent, since they are on the same indifference curve. The same is true for situations $\mathrm{B}$ and $\mathrm{B}$ ' for individual $j$. Yet, given that A' and B' are situations with perfect health, it makes sense to compare them in terms of income - the healthy-equivalent incomes for the two individuals are then $y_{i}^{*}$ and $y_{j}^{*}$ respectively.

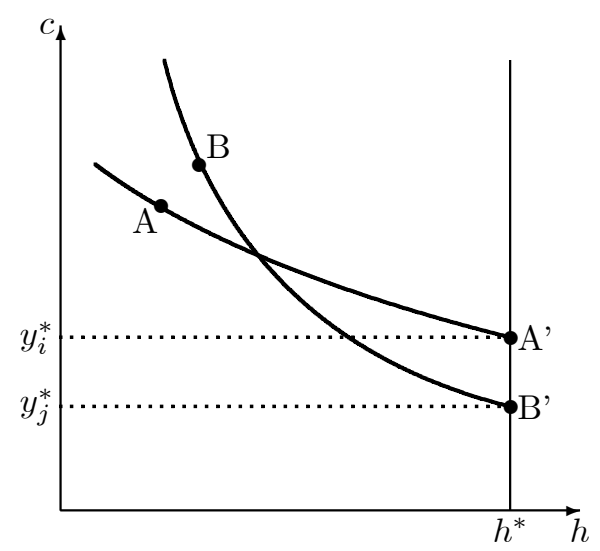

Figure 8: Healthy-equivalent income

It is clear that the computation of equivalent incomes at any given allocation requires knowing people's indifference curves at this allocation, which corresponds exactly to the second solution to Arrow's impossibility that was introduced before. This approach respects preferences more than subjective welfarism. Indeed, offering an individual a situation that he himself prefers necessarily raises his equivalent income. Moreover, two individuals with the same preferences will necessarily rank their own situations in a way that is congruent with their equivalent incomes. The healthy-equivalent income is obviously not the only way to respect preferences in this way. One could take health instead of income as the numeraire, or one could take another level than good health as the reference in the computation of the equivalent income. Taking another health level as reference is not very interesting, however, as it would not imply the nice property that we can compare individuals with good health only on the basis of their incomes and that, in contrast, comparisons of individuals with less than perfect health require taking account of preferences. For a given level of bad health, indeed, an individual with more income than another is not necessarily in a better situation if his preferences care much more about health. Moreover, if a low level of health was taken as the reference, one might find individuals in good situations who would never accept trading their situation for such a low health, even with an unlimited amount of income. In 
contrast, everyone considers good health to be at least as good as his current health, so that equivalent incomes are always lower than current incomes. One might theoretically imagine people whose situation is so bad that a good health with a null income would be better than their current situation, but this is rather far-fetched, as a null income does not sustain life. Moreover, even if one finds people in such a situation, although they cannot be ranked in terms of equivalent incomes, at least they are clearly ranked as disadvantaged with respect to all people with a positive equivalent income. ${ }^{42}$

Once equivalent incomes are adopted as the measure of individual well-being, they can be used to compute inequality in well-being and, as for the happiness measures, this analysis can integrate the concerns for (inequity in) health or health care and for the income consequences of out-of-pocket payments for health care. Of course, the approach is rather demanding as far as data are concerned. Indeed, ideally it requires an estimation of the joint distribution of preferences and individual income-health situations. This is rather hard to obtain. Even specific surveys asking direct questions about preferences can hardly do more than estimating the average preferences for subgroups of the population. Note, however, that the difference between actual income $y_{i}$ and equivalent income $y_{i}^{*}$ is basically the willingness-to-pay of the individual to be in perfect health, i.e., $y_{i}^{*}=y_{i}-W T P_{i}$. Once we have observations for $y_{i}$ and for this willingness-to-pay, we can approximate $y_{i}^{*}$.

Information about willingness-to-pay can be derived from different sources. First, one can use the estimates that are derived from the happiness equations, as described in the previous subsection. Graham et al. (2010) use this information to adjust the mean incomes of different countries for the cost of health problems. Note that in this approach one only needs the marginal rates of substitution beween income and health $\left(\partial f / \partial h_{i}\right) /\left(\partial f / \partial y_{i}\right)$, that are invariant under monotonic transformations of $f($.$) . These measures$ will therefore be much less sensitive to the adaptation issue, since it can be assumed that adaptation in the first place impacts on the measured level of happiness rather than on the orientation of preferences. Second, one can develop a contingent valuation approach to measure the willingness-to-pay for perfect health in a survey (see Fleurbaey et al. 2010). Third, one can calibrate meaningful willingness-to-pay measures using information from different sources, including observed choice behavior. This approach is followed by Becker et al. (2005) to compute for 96 countries equivalent growth rates incorporating

\footnotetext{
${ }^{42}$ One might think of a dominance principle imposing that more income and a better health always means a better situation, independently of preferences (e.g., Sen 1985). This would imply that in Figure 8 individual $j$ in situation B is better of than individual $i$ in situation A. However, this approach implies that preferences play no role in the evaluation of a situation and this goes against the basic motivation that is explored in this subsection.
} 
the monetary equivalent of improved life expectancy and by Fleurbaey and Gaulier (2009) to compare the living standards of 24 OECD countries on the basis of a broad list of dimensions including life expectancy. Not only does this recent work show that the computation of equivalent incomes is feasible, it also confirms that health is an essential element of well-being and that explicitly taking it into account may have a strong impact on inequality measures. As an example, Becker et al. (2005) estimate for the period 1960-2000 an average yearly growth of equivalent income ${ }^{43}$ of 4.1 percent for the poorest 50 percent of countries in 1960, of which 1.7 percentage points are due to health, as opposed to a growth of 2.6 percent for the richest 50 percent of countries, of which only 0.4 percentage points are due to health.

It is interesting here to make a connection with the different views on equality of opportunity that have been introduced in the previous section. We there made the distinction between the so-called "choice" and "preference" approaches. It must now have become clear that the concept of equivalent income is very much in line with the preference approach. Respecting individual preference variation in the measurement of well-being is closely related to holding people responsible for their preferences. In theory it is not very difficult to enrich the approach by introducing preferences over lifestyles, in addition to income and health, in order to address the issues of personal responsibility for health that have been examined in the previous sections. This appears a promising alley for future research.

We conclude by mentioning an important challenge to the ideal of respecting individual preferences. The equivalent income approach assumes that it is possible to get reliable information about the genuine preferences of individuals on what is valuable in life and, even more basically, that these individual preferences are well-defined. Now that behavioral studies have uncovered many ways in which individual preferences may deviate from this ideal, the equivalent income measure cannot be implemented without caution. One may even think that the underlying theory should be refined in order to accommodate the most common departures from rationality that have been revealed in the behavioral literature.

\section{Conclusion}

Distributional issues play an important role in all social discussions on health (care) policies. If economists want to give relevant advice, they need a coherent normative framework for policy evaluation. Such normative framework can also guide empirical research by suggesting what the important facts are and possibly what causal relationships should be analysed. In this respect, the recent health economic litera-

\footnotetext{
${ }^{43}$ They use the terminology "full income".
} 
ture has produced many interesting insights by focusing on specific issues, such as that of socioeconomic inequalities in health, inequities in health care delivery, racial disparities and the income consequences of out-of-pocket payments. It would be useful, however, to integrate these partial insights into a broader framework.

First, concern about inequity in health and in health care should not be restricted to specific characteristics such as socioeconomic status or race. At a more fundamental level "horizontal equity" is only achieved when all individuals with the same needs and the same preferences get the same level of care and all factors leading to deviations from this ideal should be considered together. Moreover, the issue of horizontal equity is intricately linked to that of vertical equity. In the same way, equity in health will only be achieved if the remaining health inequality can be fully ascribed to factors for which individuals can be held responsible. Of course, opinions differ about what these factors are exactly, e.g., to what extent individuals have to bear the health consequences of their choice of lifestyle.

Second, from the distributional point of view, we are ultimately interested in overall well-being. Despite the crucial importance of health, it is not the only relevant dimension. As the literature on equity in finance shows, it is at least necessary to be explicit about the trade-offs between health on the one hand and consumption on the other hand - but clearly, a sufficiently rich concept of well-being will have to include other dimensions than health and consumption too.

Recent developments in the welfare economic literature offer a promising framework to tackle both sets of questions. Inequity in health and in health care can be seen as a prime example of inequality of opportunity - and the theory of fair allocation now provides a host of interesting approaches to model that concept and gives clear insights into the normative implications of these different approaches. Much progress has also been made in the quest for an overall concept of well-being. Not only does it now seem possible to measure subjective well-being, we also understand better the ethical limitations of that notion and interesting alternatives (such as the equivalent income) have been developed. In this chapter we have tried to show how this literature relates to the specific issues that have been analysed by health economists. It turns out that there is not really a conflict between them: quite the contrary, the typical health economic approaches can be seen as useful building blocks in the broader exercise. Moreover, further thinking along these lines will also make it possible to better see how the operational question of inequity measurement relates to deeper social philosophical questions on justice and individual responsibility, and it suggests why and where good econometrics is needed for the estimation of structural models. Building these bridges may enrich the theoretical content of the health economic applications 
and yield useful insights into the applicability of welfare economic theories. 


\section{References}

Abul Naga, R., and P.-Y. Geoffard. 2006. Decomposition of bivariate inequality indices by attributes. Economics Letters 90, no. 3: 362-67.

Agency for Healthcare Research and Quality. 2010. National Healthcare Disparities Report 2009. Rockville, MD: US Department of Health and Human Services: AHRQ Publication no. 10-0004, available at http://www.ahrq.gov/qual/measurix.htm\#disparity.

Allanson, P., U.-G. Gerdtham, and D. Petrie. 2010. Longitudinal analysis of income-related health inequality. Journal of Health Economics 29, no. 1: 78-86.

Anand, P., and P. Dolan. 2005. Equity, capabilities and health. Social Science and Medicine 60: 219-22.

Anderson, E. 1999. What is the point of equality? Ethics 109: 287-337.

Armstrong, K., C. Hughes-Halbert, and D. Asch. 2006. Patient preferences can be misleading as explanations for racial disparities in health care. Archives of Internal Medicine 166, no. 8: 950-954.

Arneson, R. 1989. Equality and equal opportunity for welfare. Philosophical Studies 56: 77-93.

Arrow, K. 1951. Social choice and individual values. New York: Wiley.

-1971. A utilitarian approach to the concept of equality in public expenditures. Quarterly Journal of Economics 85: 409-15.

Ashton, C., P. Haidet, D. Paterniti, T. Collins, H. Gordon, K. O’Malley, L. Petersen, B. Sharf, M. SuarezAlmazor, N. Wray, and R. Street. 2003. Racial and ethnic disparities in the use of health services: bias, preferences, or poor communication? Journal of General Internal Medicine 18, no. 2: 146-52.

Atkinson, A., and F. Bourguignon. 1982. The comparison of multi-dimensioned distributions of economic status. Review of Economic Studies 49: 183-201.

— 1987. Income distribution and differences in needs. Arrow and the foundations of economic policy. ed. G. Feiwel, 350-370. London: Macmillan.

Ayanian, J., P. Cleary, J. Weissman, and A. Epstein. 1999. The effect of patients' preferences on racial differences in access to renal transplantation. New England Journal of Medicine 341: 1661-69.

Bago d'Uva, T., A. Jones, and E. van Doorslaer. 2009. Measurement of horizontal inequity in health care utilisation using European panel data. Journal of Health Economics 28, no. 2: 280-289.

Balia, S. , and A. Jones. 2011. Catching the habit: a study of inequality of opportunity in smoking-related mortality. Journal of the Royal Statistical Society A 174, no. 1: 175-94.

Becker, G., T. Philipson, and R. Soares. 2005. The quantity and quality of life and the evolution of world 
inequality. American Economic Review 95, no. 1: 277-91.

Blackorby, C., and D. Donaldson. 1988. Money metric utility: harmless normalization? Journal of Economic Theory 46, no. 1: 120-129.

Bleichrodt, H., and E. van Doorslaer. 2006. A welfare economics foundation for health inequality measurement. Journal of Health Economics 25: 945-57.

Blinder, A. 1973. Wage discrimination: reduced form and structural estimates. Journal of Human Resources 8: $436-55$.

Bollinger, B., P. Leslie, and A. Sorensen. 2011. Calorie posting in chain restaurants. American Economic Journal: Economic Policy 3: 91-128.

Bommier, A., and G. Stecklov. 2002. Defining health inequality: why Rawls succeeds where social welfare theory fails. Journal of Health Economics 21: 497-513.

Bourguignon, F. 1989. Family size and social utility: income distribution dominance criteria. Journal of Econometrics 42: 67-80.

Case, A., A. Fertig, and C. Paxson. 2005. The lasting impact of childhood health and circumstance. Journal of Health Economics 24: 365-89.

Case, A., D. Lubotsky, and C. Paxson. 2002. Economic status and health in childhood: the origins of the gradient. American Economic Review 92, no. 5: 1308-34.

Chou, S.-Y., I. Rashad, and M. Grossman. 2008. Fast-food restaurant advertising on television and its influence on childhood obesity. Journal of Law and Economics 51, no. 4: 599-618.

Cisse, B., S. Luchini, and J. P. Moatti. 2007. Progressivity and horizontal equity in health care finance and delivery: what about Africa? Health Policy 80, no. 1: 51-68.

Clarke, P., U.-G. Gerdtham, M. Johannesson, K. Bingefors, and L. Smith. 2002. On the measurement of relative and absolute income-related health inequality. Social Science and Medicine 55, no. 11: 1923-28.

Cohen, G. 1989. On the currency of egalitarian justice. Ethics 99: 906-44.

Cook, B., T. McGuire, K. Lock, and A. Zaslavsky. 2010. Comparing methods of racial and ethnic disparities measurement across different settings of mental health care. Health Services Research 45, no. 3: 825-47.

Cook, B., T. McGuire, E. Meara, and A. Zaslavsky. 2009. Adjusting for health status in non-linear models of health care disparities. Health Services and Outcomes Research Methodology 9: 1-21.

Currie, J. 2009. Healthy, wealthy and wise: socioeconomic status, poor health in childhood, and human capital development. Journal of Economic Literature 47, no. 1: 87-122. 
Currie, J., and M. Stabile. 2003. Socioeconomic status and child health: why is the relationship stronger for older children? American Economic Review 93, no. 5: 1813-23.

Cutler, D., and A. Lleras-Muney. 2010. Understanding differences in health behaviors by education. Journal of Health Economics : forthcoming.

Daniels, N. 1985. Just health care. Cambridge: Cambridge University Press.

—_ 2008. Just health. Meeting health needs fairly. Cambridge: Cambridge University Press.

De Graeve, D., and T. Van Ourti. 2003. The distributional impact of health financing in Europe: a review. The World Economy 26: 1459-79.

Deaton, A. 2011. What does the empirical evidence tell us about the injustice of health inequalities? Princeton University: Mimeo.

Deaton, A., and J. Muellbauer. 1980. Economics and consumer behavior. Cambridge: Cambridge University Press.

Duclos, J.-Y., and D. Echevin. 2011. Health and income: a robust comparison of Canada and the US. Journal of Health Economics 30: 293-302.

Dutta, I. , P. Pattanaik, and Y. Xu. 2003. On measuring deprivation and the standard of living in a multidimensional framework on the basis of aggregate data. Economica 70: 197-221.

Dworkin, R. 1981a. What is equality? Part 1: Equality of welfare. Philosophy and Public Affairs 10: $185-246$.

—_ 1981b. What is equality? Part 2: Equality of resources. Philosophy and Public Affairs 10: 283-345.

—_. 2000. Sovereign virtue. Cambridge: Cambridge University Press.

Erreygers, G. 2009a. Correcting the concentration index. Journal of Health Economics 28: 504-15. 2009b. Correcting the concentration index: a reply to Wagstaff. Journal of Health Economics 28: 521-24.

2009c. Can a single indicator measure both attainment and shortfall inequality? Journal of Health Economics 28: 885-93.

Erreygers, G., and T. Van Ourti. 2010. Measuring socioeconomic inequality in health, health care and health financing by means of rank-dependent indices: a recipe for good practice. Tinbergen Institute: Discussion Paper TI 2010-076/3.

Ferrer-i-Carbonell, A., and B. Van Praag. 2002. The subjective costs of health losses due to chronic diseases. An alternative model for monetary appraisal. Health Economics 11: 709-22.

Fleurbaey, M. 2005. Health, wealth and fairness. Journal of Public Economic Theory 7, no. 2: 253-84. 
—_. 2006. Health, equity and social welfare. Annales d'Economie et Statistique 83-84: 21-60.

—_. 2008. Fairness, responsibility and welfare. Oxford: Oxford University Press.

. 2009. Beyond GDP: the Quest for a Measure of Social Welfare. Journal of Economic Literature 47, no. 4: 1029-75.

2010. Assessing risky social decisions. Journal of Political Economy 118: 649-80.

Fleurbaey, M., and G. Gaulier. 2009. International comparisons of living standards by equivalent incomes. Scandinavian Journal of Economics 111, no. 3: 597-624.

Fleurbaey, M., S. Luchini, C. Muller, and E. Schokkaert. 2010. Equivalent income and the economic evaluation of health care. CORE-Discussion Paper 2010/6.

Fleurbaey, M., and F. Maniquet. 2011. A theory of fairness and social welfare. Cambridge: Cambridge University Press.

Fleurbaey, M., and E. Schokkaert. 2009. Unfair inequalities in health and health care. Journal of Health Economics 28, no. 1: 73-90.

Fleurbaey, M., and A. Trannoy. 2003. The impossibility of a Paretian egalitarian. Social Choice and Welfare 21, no. 2: 243-63.

Flores, G., J. Krishnakumar, O. O’Donnell, and E. van Doorslaer. 2008. Coping with health-care costs: implications for the measurement of catastrophic expenditures and poverty. Health Economics 17, no. 12: 1393412.

Frey, B., and A. Stutzer. 2002. What can economists learn from happiness research? Journal of Economic Literature 40, no. 2: 402-35.

Gaertner, W., and E. Schokkaert. 2011. Empirical social choice. Cambridge: Cambridge University Press (forthcoming).

Gajdos, T., and J. Weymark. 2005. Multidimensional generalized Gini indices. Economic Theory 26: 471-96.

Graham, C. 2008. Happiness and health: lessons - and questions - for policy. Health Affairs 27, no. 1: $72-81$.

Graham, C., L. Higuera, and E. Lora. 2011. Which health conditions cause the most unhappiness? Health Economics : forthcoming.

Gravel, N., P. Moyes. 2011. Bidimensional inequalities with an ordinal variable. In M. Fleurbaey, M. Salles, J.A. Weymark (eds.), Social Ethics and Normative Economics, Berlin: Springer.

Gravelle, H. 2003. Measuring income related inequality in health: standardisation and the partial concentration index. Health Economics 12: 803-19. 
Groot, W. , and H. Maassen van den Brink. 2006. The compensating income variation of cardiovascular disease. Health Economics 15: 1143-48.

Groot, W. , Maassen van den Brink, H. and E. Plug. 2004. Money for health: the equivalent variation of cardiovascular disease. Health Economics 13: 859-72.

Hansson, B. 1973. The independence condition in the theory of social choice. Theory and Decision 4: 25-49.

Hardy, G., J. Littlewood, and G. Polya. 1952. Inequalities. Cambridge: Cambridge University Press.

Harsanyi, J. 1977. Rational behavior and bargaining equilibrium in games and social situations. Cambridge: Cambridge University Press.

Hausman, D. 2007. What's wrong with health inequalities? Journal of Political Philosophy 15, no. 1: 46-66. Institute of Medicine. 2002. Unequal treatment: confronting racial and ethnic disparities in health care. Washington, DC: National Academy Press.

Jenkins, S., and P. Lambert. 1993. Ranking income distributions when needs differ. Review of Income and Wealth 39: 337-56.

Jones, A. , and A. Lopez Nicolas. 2004. Measurement and explanation of socioeconomic inequality in health with longitudinal data. Health Economics 13: 1015-30.

Jusot, F. , S. Tubeuf, and A. Trannoy. 2010. Effort or circumstances: does the correlation matter for inequality of opportunity in health? IRDES, Paris: Working Paper DT 33.

Kakwani, N., A. Wagstaff, and E. van Doorslaer. 1997. Socioeconomic inequalities in health: measurement, computation, and statistical inference. Journal of Econometrics 77: 87-103.

Kawachi, I., N. Daniels, and D. Robinson. 2005. Health disparities by race and class: why both matter. Health Affairs 24, no. 2: 343-52.

King, M. 1983. Welfare analysis of tax reforms using household data. Journal of Public Economics 23: $183-214$.

Kolm, S.-Chr. 1977. Multidimensional egalitarianisms. Quarterly Journal of Economics 91, no. 1: 1-13.

Koolman, X., and E. van Doorslaer. 2004. On the interpretation of a concentration index of inequality. Health Economics 13: 649-56.

Lambert, P., Zheng, and B. 2011. On the consistent measurement of achievement and shortfall inequality. Journal of Health Economics 30, no. 1: 214-19.

Layard, R. 2005. Happiness: lessons from a new science. Allan Lane.

Le Grand, J. 1987. Inequalities in health: some international comparisons. European Economic Review 31, no. $1 / 2: 182-91$. 


\section{- 1991. Equity and choice. London: Harper Collins Academic.}

Lefranc, A., N. Pistolesi, and A. Trannoy. 2009. Equality of opportunity and luck: definitions and testable conditions, with an application to income in France. Journal of Public Economics 93: 1189-207.

Levy, H., and J. Paroush. 1974. Towards multivariate efficiency criteria. Journal of Economic Theory 7: $129-42$.

Lippert-Rasmussen, K. 2001. Egalitarianism, option luck, and responsibility. Ethics.111: 548-579.

Loewenstein, G., and P. Ubel. 2008. Hedonic adaptation and the role of decision and experienced utility in public policy. Journal of Public Economics 92: 1795-810.

Lu, R., G. Leung, S. Kwon, K. Tin, E. van Doorslaer, and O. O'Donnell. 2007. Horizontal equity in health care utilization — evidence from three high income Asian economies. Social Science and Medicine 64: 199-212.

Milanovic, B. 1997. A simple way to calculate the Gini coefficient and some implications. Economics Letters 56: $45-49$.

Morris, S., M. Sutton, and H. Gravelle. 2005. Inequity and inequality in the use of health care in England: an empirical investigation. Social Science and Medicine 60: 1251-66.

Moyes, P. 1999. Comparison of heterogeneous distributions and dominance criteria. Economie et Prévision 138-139: 125-146.

Muller, C., and A. Trannoy. 2003. Multidimensional inequality comparisons: a compensation perspective. Mimeo

O’Donnell, O., E. van Doorslaer, R. Rannan-Eliya, A. Somanathan, S. R. Adhikari, B. Akkazieva, D. Harbianto, C. Carg, P. Hanvoravongchai, A. Herrin, M. Huq, S. Ibragimova, A. Karan, S. Leung G. Kwon, J. R. Lu, Y. Ohkusa, B. R. Pande, R. Racelis, K. Tin, K. Tisayaticom, L. Trisnantoro, Q. Wan, B-M. Yang, and Y. Zhao. 2008. Who pays for health care in Asia? Journal of Health Economics 27: 460-475.

O'Donnell, O., E. van Doorslaer, A. Wagstaff, and M. Lindelow. 2008. Analyzing health equity using household survey data. Washington, D.C.: World Bank.

Oaxaca, R. 1973. Male-female wage differentials in urban labor markets. International Economic Review 14, no. 3: 693-709.

Pazner, E., and D. Schmeidler. 1978. Egalitarian equivalent allocations: a new concept of economic equity. Quarterly Journal of Economics 92: 671-87.

Phillips, A. 2006. 'Really' equal: opportunities and autonomy. Journal of Political Philosophy 14: 18-32.

Rawls, J. 1971. A theory of justice. Cambridge, Mass.: Harvard University Press. 
Rawls, J. 1982. Social unity and primary goods. Utilitarianism and beyond. eds. A. Sen, and B. Williams , 159-85. Cambridge: Cambridge University Press.

Richard, S. 1975. Multivariate risk aversion, utility independence and separable utility functions. Management Science 22: 12-21.

Roemer, J. 1985. Equality of talent. Economics and Philosophy 1: 151-87.

— 1993. A pragmatic theory of responsibility for the egalitarian planner. Philosophy and Public Affairs 22: $146-66$.

—_. 1998. Equality of opportunity. Cambridge, Mass.: Harvard University Press.

- 2002. Equality of opportunity: a progress report. Social Choice and Welfare 19, no. 2: 455-71.

Rosa Dias, P. 2009. Inequality of opportunity in health: evidence from a UK cohort study. Health Economics 18: $1057-74$.

—_. 2010. Modelling opportunity in health under partial observability of circurmstances. Health Economics 19: 252-64.

Rosa Dias, P., and A. Jones. 2007. Giving equality of opportunity a fair innings. Health Economics 16: 109-12.

Ruhm, C., and J. Cawley. 2011. Health behaviors. Handbook of Health Economics, Volume 2. Amsterdam: Elsevier: forthcoming.

Samuelson, P. 1974. Complementarity: an essay on the 40th anniversary of the Hicks-Allen revolution in demand theory. Journal of Economic Literature 12: 1255-89.

Samuelson, P., and S. Swamy. 1974. Invariant economic index numbers and canonical duality: survey and synthesis. American Economic Review 64, no. 4: 566-93.

Sandy, R., G. Liu, J. Ottensmann, R. Tchernis, J. Wilson, and O. Ford. 2009. Studying the child obesity epidemic with natural experiments. NBER: Working Paper No. 14989.

Schneider, P., and K. Hanson. 2006. Horizontal equity in utilisation of care and fairness of health financing: a comparison of micro-health insurance and user fees in Rwanda. Health Economics 15, no. 1: 19-31.

Schokkaert, E., and C. Van de Voorde. 2004. Risk selection and the specification of the conventional risk adjustment formula. Journal of Health Economics 23: 1237-59.

2009. Direct versus indirect standardization in risk adjustment. Journal of Health Economics 28: $361-74$.

. 2011. User charges. Oxford Handbook on Health Economics. eds. S. Glied, and P. Smith, 329-353.

Oxford: Oxford University Press. 
Segall, S. 2010. Health, luck and justice. Princeton: Princeton University Press.

Sen, A. 1970. Collective choice and social welfare. San Francisco: Holden Day.

1985. Commodities and capabilities. Amsterdam: North-Holland.

—_ 1999. Development as freedom. New York: Knopf.

__ 2002. Why health equity? Health Economics 11: 659-66.

Shorrocks, A. 1982. Inequality decomposition by factor components. Econometrica 50: 193-211.

Sutton, M. 2002. Vertical and horizontal aspects of socio-economic inequity in general practitioner contacts in Scotland. Health Economics 11: 537-49.

Tobin, J. 1970. On limiting the domain of inequality. Journal of Law and Economics 13: 263-78.

Trannoy, A. 2006. Multidimensional egalitarianism and the dominance approach: a lost paradise? Inequality and economic integration. eds. F. Farina, and E. Savaglio, 284-302. London: Routledge.

Trannoy, A., S. Tubeuf, F. Jusot, and M. Devaux. 2010. Inequality of opportunities in health in France: a first pass. Health Economics 19, no. 8: 921-38.

Tsui, K.-Y. 1995. Multidimensional generalizations of the relative and absolute inequality indices: the Atkinson-Kolm-Sen approach. Journal of Economic Theory 67: 251-65.

Tsui, K.-Y. 1999. Multidimensional inequality and multidimensional generalized entropy measures: An axiomatic derivation. Social Choice and Welfare 16: 145-57.

Vallentyne, P. 2002. Brute luck, option luck and equality of initial opportunities. Ethics 112: 529-57.

Van de Poel, E., O. O’Donnell, and E. van Doorslaer. 2007. Are urban children really healthier? Evidence from 47 developing countries. Social Science and Medicine 65: 1986-2003.

Van den Berg, G., M. Lindeboom, and F. Portrait. 2006. Economic conditions early in life and individual mortality. American Economic Review 96, no. 1: 290-302.

van Doorslaer, E., and A. Jones. 2003. Inequalities in self-reported health: validation of a new approach to measurement. Journal of Health Economics 22: 61-87.

van Doorslaer, E., and X. Koolman. 2004. Explaining the differences in income-related health inequalities across European countries. Health Economics 13: 609-28.

van Doorslaer, E., X. Koolman, and A. Jones. 2004. Explaining income-related inequalities in doctor utilisation in Europe. Health Economics 13: 629-47.

van Doorslaer, E., O. O’Donnell, R. Rannan-Eliya, A. Somanathan, S. Adhikari, C. Garg, D. Harbianto, A. Herrin, M. Huq, S. Ibragimova, A. Karan, T.-J. Lee, G. Leung, J. Lu, C. Ng, B. Pande, R. Racelis, S. Tao, K. Tin, K. Tisayaticom, L. Trisnantoro, C. Vasavid, and Y. Zhao. 2007. Catastrophic payments for health care in 
Asia. Health Economics 16: 1159-84.

van Doorslaer, E., O. O’Donnell, R. Rannan-Eliya, A. Somanathan, S. Adhikari, C. Garg, D. Harbianto, A. Herrin, M. Huq, S. Ibragimova, A. Karan, C. Ng, B. Pande, R. Racelis, S. Tao, K. Tin, K. Tisayaticom, L. Trisnantoro, C. Vasavid, and Y. Zhao. 2006. Effect of payments for health care on poverty estimates in 11 countries in Asia: an analysis of household survey data. The Lancet 368: 1357-64.

van Doorslaer, E., and T. Van Ourti. 2011. Measuring inequality and inequity in health and health care. Oxford Handbook on Health Economics. eds. S. Glied, and P. Smith, 837-869. Oxford: Oxford University Press. van Doorslaer, E., A. Wagstaff, H. van der Burg, T. Christiansen, G. Citoni, R. Di Biase, U.-G. Gerdtham, M. Gerfin, L. Gross, U. Häkinnen, J. John, P. Johnson, J. Klavus, C. Lachaud, J. Lauritsen, R. Leu, B. Nolan, J. Pereira, C. Propper, F. Puffer, L. Rochaix, M. Rodriguez, M. Schellhorn, G. Sundberg, and O. Winkelhake. 1999. The redistributive effect of health care finance in twelve OECD countries. Journal of Health Economics 18: 291-313.

van Doorslaer, E., A. Wagstaff, H. van der Burg, T. Christiansen, D. De Graeve, I. Duchesne, Ulf-G. Gerdtham, M. Gerfin, J. Geurts, L. Gross, U. Häkkinen, J. John, J. Klavus, R. Leu, B. Nolan, O. O’Donnell, C. Propper, F. Puffer, M. Schellhorn, G. Sundberg, and O. Winkelhake. 2000. Equity in the delivery of health care in Europe and the US. Journal of Health Economics 19: 553-83.

Van Ourti, T. 2004. Measuring horizontal inequity in Belgian health care using a Gaussian random effects two part count data model. Health Economics 13: 705-24.

Wagstaff, A. 2000. Socioeconomic inequalities in child mortality: comparisons across nine developing countries. Bulletin of the World Health Organization 78, no. 1: 19-29.

2002. Inequality aversion, health inequalities and health achievement. Journal of Health Economics 21: $627-41$.

- 2005. The bounds of the concentration index when the variable of interest is binary, with an application to immunization inequality. Health Economics 14: 429-32.

—_. 2008. Measuring financial protection in health. World Bank: Policy Research Working Paper 4554.

—_ 2009. Correcting the concentration index: a comment. Journal of Health Economics 28: 516-20.

Wagstaff, A., and E. van Doorslaer. 2000. Measuring and testing for inequity in the delivery of health care. Journal of Human Resources 35, no. 4: 716-33.

- 2003. Catastrophe and impoverishment in paying for health care: with applications to Vietnam 1993-98. Health Economics 12, no. 11: 921-33.

2004. Overall versus socioeconomic health inequality: a measurement framework and two empirical 
illustrations. Health Economics 13: 297-301.

2000. Equity in health care finance and delivery. Handbook of Health Economics, Vol. 1B. eds. A. Culyer, and J. Newhouse, 1803-62. Amsterdam: Elsevier (North-Holland).

Wagstaff, A., E. van Doorslaer, and N. Watanabe. 2003. On decomposing the causes of health sector inequalities with an application to malnutrition inequalities in Vietnam. Journal of Econometrics 112: 207-23.

Weymark, J. 2006. The normative approach to the measurement of multidimensional inequality. Inequality and economic integration. eds. F. Farina, and E. Savaglio, 303-28. London: Routledge.

Xu, K., D. Evans, G. Carrin, A. Aguilar-Rivera, P. Musgrove, and T. Evans. 2007. Protecting households from catastrophic health spending. Social Science and Medicine 26, no. 4: 972-83.

Xu, K., D. Evans, K. Kawabata, R. Zeramdini, J. Klavus, and C. Murray. 2003. Household catastrophic health expenditure: a multicountry analysis. The Lancet 362, no. 111-117. 


\section{Recent titles \\ CORE Discussion Papers}

2010/71. Carlotta BALESTRA, Thierry BRECHET and Stéphane LAMBRECHT. Property rights with biological spillovers: when Hardin meets Meade.

2010/72. Olivier GERGAUD and Victor GINSBURGH. Success: talent, intelligence or beauty?

2010/73. Jean GABSZEWICZ, Victor GINSBURGH, Didier LAUSSEL and Shlomo WEBER. Foreign languages' acquisition: self learning and linguistic schools.

2010/74. Cédric CEULEMANS, Victor GINSBURGH and Patrick LEGROS. Rock and roll bands, (in)complete contracts and creativity.

2010/75. Nicolas GILLIS and François GLINEUR. Low-rank matrix approximation with weights or missing data is NP-hard.

2010/76. Ana MAULEON, Vincent VANNETELBOSCH and Cecilia VERGARI. Unions' relative concerns and strikes in wage bargaining.

2010/77. Ana MAULEON, Vincent VANNETELBOSCH and Cecilia VERGARI. Bargaining and delay in patent licensing.

2010/78. Jean J. GABSZEWICZ and Ornella TAROLA. Product innovation and market acquisition of firms.

2010/79. Michel LE BRETON, Juan D. MORENO-TERNERO, Alexei SAVVATEEV and Shlomo WEBER. Stability and fairness in models with a multiple membership.

2010/80. Juan D. MORENO-TERNERO. Voting over piece-wise linear tax methods.

2010/81. Jean HINDRIKS, Marijn VERSCHELDE, Glenn RAYP and Koen SCHOORS. School tracking, social segregation and educational opportunity: evidence from Belgium.

2010/82. Jean HINDRIKS, Marijn VERSCHELDE, Glenn RAYP and Koen SCHOORS. School autonomy and educational performance: within-country evidence.

2010/83. Dunia LOPEZ-PINTADO. Influence networks.

2010/84. Per AGRELL and Axel GAUTIER. A theory of soft capture.

2010/85. Per AGRELL and Roman KASPERZEC. Dynamic joint investments in supply chains under information asymmetry.

2010/86. Thierry BRECHET and Pierre M. PICARD. The economics of airport noise: how to manage markets for noise licenses.

2010/87. Eve RAMAEKERS. Fair allocation of indivisible goods among two agents.

2011/1. Yu. NESTEROV. Random gradient-free minimization of convex functions.

2011/2. Olivier DEVOLDER, François GLINEUR and Yu. NESTEROV. First-order methods of smooth convex optimization with inexact oracle.

2011/3. Luc BAUWENS, Gary KOOP, Dimitris KOROBILIS and Jeroen V.K. ROMBOUTS. A comparison of forecasting procedures for macroeconomic series: the contribution of structural break models.

2011/4. Taoufik BOUEZMARNI and Sébastien VAN BELLEGEM. Nonparametric Beta kernel estimator for long memory time series.

2011/5. Filippo L. CALCIANO. The complementarity foundations of industrial organization.

2011/6. Vincent BODART, Bertrand CANDELON and Jean-François CARPANTIER. Real exchanges rates in commodity producing countries: a reappraisal.

2011/7. Georg KIRCHSTEIGER, Marco MANTOVANI, Ana MAULEON and Vincent VANNETELBOSCH. Myopic or farsighted? An experiment on network formation.

2011/8. Florian MAYNERIS and Sandra PONCET. Export performance of Chinese domestic firms: the role of foreign export spillovers.

2011/9. Hiroshi UNO. Nested potentials and robust equilibria.

2011/10. Evgeny ZHELOBODKO, Sergey KOKOVIN, Mathieu PARENTI and Jacques-François THISSE. Monopolistic competition in general equilibrium: beyond the CES.

2011/11. Luc BAUWENS, Christian HAFNER and Diane PIERRET. Multivariate volatility modeling of electricity futures.

2011/12. Jacques-François THISSE. Geographical economics: a historical perspective. 


\section{Recent titles}

\section{CORE Discussion Papers - continued}

2011/13. Luc BAUWENS, Arnaud DUFAYS and Jeroen V.K. ROMBOUTS. Marginal likelihood for Markov-switching and change-point GARCH models.

2011/14. Gilles GRANDJEAN. Risk-sharing networks and farsighted stability.

2011/15. Pedro CANTOS-SANCHEZ, Rafael MONER-COLONQUES, José J. SEMPERE-MONERRIS and Oscar ALVAREZ-SANJAIME. Vertical integration and exclusivities in maritime freight transport.

2011/16. Géraldine STRACK, Bernard FORTZ, Fouad RIANE and Mathieu VAN VYVE. Comparison of heuristic procedures for an integrated model for production and distribution planning in an environment of shared resources.

2011/17. Juan A. MAÑEZ, Rafael MONER-COLONQUES, José J. SEMPERE-MONERRIS and Amparo URBANO Price differentials among brands in retail distribution: product quality and service quality.

2011/18. Pierre M. PICARD and Bruno VAN POTTELSBERGHE DE LA POTTERIE. Patent office governance and patent system quality.

2011/19. Emmanuelle AURIOL and Pierre M. PICARD. A theory of BOT concession contracts.

2011/20. Fred SCHROYEN. Attitudes towards income risk in the presence of quantity constraints.

2011/21. Dimitris KOROBILIS. Hierarchical shrinkage priors for dynamic regressions with many predictors.

2011/22. Dimitris KOROBILIS. VAR forecasting using Bayesian variable selection.

2011/23. Marc FLEURBAEY and Stéphane ZUBER. Inequality aversion and separability in social risk evaluation.

2011/24. Helmuth CREMER and Pierre PESTIEAU. Social long term care insurance and redistribution.

2011/25. Natali HRITONENKO and Yuri YATSENKO. Sustainable growth and modernization under environmental hazard and adaptation.

2011/26. Marc FLEURBAEY and Erik SCHOKKAERT. Equity in health and health care.

\section{Books}

P. VAN HENTENRYCKE and L. WOLSEY (eds.) (2007), Integration of AI and OR techniques in constraint programming for combinatorial optimization problems. Berlin, Springer.

P-P. COMBES, Th. MAYER and J-F. THISSE (eds.) (2008), Economic geography: the integration of regions and nations. Princeton, Princeton University Press.

J. HINDRIKS (ed.) (2008), Au-delà de Copernic: de la confusion au consensus ? Brussels, Academic and Scientific Publishers.

J-M. HURIOT and J-F. THISSE (eds) (2009), Economics of cities. Cambridge, Cambridge University Press.

P. BELLEFLAMME and M. PEITZ (eds) (2010), Industrial organization: markets and strategies. Cambridge University Press.

M. JUNGER, Th. LIEBLING, D. NADDEF, G. NEMHAUSER, W. PULLEYBLANK, G. REINELT, G. RINALDI and L. WOLSEY (eds) (2010), 50 years of integer programming, 1958-2008: from the early years to the state-of-the-art. Berlin Springer.

G. DURANTON, Ph. MARTIN, Th. MAYER and F. MAYNERIS (eds) (2010), The economics of clusters Lessons from the French experience. Oxford University Press.

J. HINDRIKS and I. VAN DE CLOOT (eds) (2011), Notre pension en heritage. Itinera Institute.

\section{CORE Lecture Series}

D. BIENSTOCK (2001), Potential function methods for approximately solving linear programming problems: theory and practice.

R. AMIR (2002), Supermodularity and complementarity in economics.

R. WEISMANTEL (2006), Lectures on mixed nonlinear programming. 